\title{
A Cascade Reaction Consisting of Pictet- Spengler-type Cyclization and Smiles Rearrangement: Application to the Synthesis of Novel Pyrrole-fused Dihydropteridines
}

Jinbao Xiang, Lianyou Zheng, Feng Chen, Qun Dang, and Xu Bai*

The Center for Combinatorial Chemistry and Drug Discovery, Jilin University, 75 Haiwai Street, Changchun, Jilin 130012, P. R. China.

xbai@jlu.edu.cn

Table of Contents

$\begin{array}{ll}\text { Experimental Section-General Considerations } & \text { S2 }\end{array}$

Experimental Details and Spectroscopic Data $\quad$ S2

$\begin{array}{ll}\text { Figure Legends } & \text { S9 }\end{array}$

$\begin{array}{ll}\text { Spectra } & \text { S14 }\end{array}$

* Tel: +86-431-85188955; Fax: +86-431-85188900; E-mail: xbai@jlu.edu.cn 


\section{Experimental Section}

General considerations: Dichloromethane (DCM) was dried with $\mathrm{P}_{2} \mathrm{O}_{5}$ and distilled. All other commercial reagents were used as received without additional purification. Melting point was uncorrected. Mass spectra and HPLC data was recorded on an 1100 LC/MS system (Agilent Technology Corporation) with Alltech ELSD 2000. The ${ }^{1} \mathrm{H}$ and ${ }^{13} \mathrm{C}$ NMR data were obtained on a Varian Mercury (300 MHz) or Varian INOVA (500 MHz) NMR spectrometer with TMS as the internal standard and $\mathrm{CDCl}_{3}$ as solvent. Multiplicities are indicated as the following: s, singlet; d, doublet; t, triplet; q, quartet; m, multiplet; dd, doubled doublet; br, broad. Coupling constants ( $J$ values) where noted are quoted in Hertz.

4,6-Dichloro-5-pyrrol-1-yl-pyrimidine 1. To a stirred solution of 4,6-dichloro-5aminopyrimidine $(15.0 \mathrm{~g}, \quad 92.02 \mathrm{mmol})$ in acetic acid $(150 \mathrm{~mL}), \quad 2,5-$ dimethoxytetrahydrofuran $(11.9 \mathrm{~mL}, 92.02 \mathrm{mmol})$ was added. The resulting solution was stirred for $1 \mathrm{~h}$ at reflux. Then acetic acid was evaporated and the residue was diluted with DCM and washed with water. The organic layers were dried over $\mathrm{MgSO}_{4}$, filtered, and concentrated in vacuo. Purification by flash column chromatography (Petroleum ether/EtOAc 20:1, v/v) afforded $15.3 \mathrm{~g}(78 \%)$ of $\mathbf{1}$ as a white solid. mp: $120-122{ }^{\circ} \mathrm{C} ;{ }^{1} \mathrm{H}$ NMR: $\delta 8.80(\mathrm{~s}, 1 \mathrm{H}), 6.74$ (t, $2 \mathrm{H}, J=2.4), 6.45$ (t, $2 \mathrm{H}, J=2.1$ ); MS (ESI): $m / z 214.0$ $\left[\mathrm{M}+\mathrm{H}^{+}\right]$

6-[N-Benzyl(2-ethanol)amino]-4-chloro-5-pyrrol-1-yl-pyrimidine 2a: To a stirred solution of 4,6-dichloro-5-pyrrol-1-yl-pyrimidine $(4.0 \mathrm{~g}, 18.78 \mathrm{mmol})$ and $\mathrm{N}$ benzylglycinol $(4.088 \mathrm{~g}, 27.05 \mathrm{mmol})$ in $n$-BuOH $(100 \mathrm{~mL})$ was added TEA $(4.05 \mathrm{~mL}$, $29.06 \mathrm{mmol}$ ). The resulting solution was stirred for $3.5 \mathrm{~h}$ at reflux. Then $n-\mathrm{BuOH}$ was evaporated and the residue was diluted with DCM and washed with water. The organic layers were dried over $\mathrm{MgSO}_{4}$, filtered, and concentrated in vacuo. Purification by flash column chromatography (Petroleum ether/EtOAc 2:1, v/v) afforded 5.667g (92\%) of 2a as a white solid. mp: $71-73{ }^{\circ} \mathrm{C} ;{ }^{1} \mathrm{H}$ NMR: $\delta 8.39(\mathrm{~s}, 1 \mathrm{H}), 7.31-7.24(\mathrm{~m}, 3 \mathrm{H}), 7.06(\mathrm{~d}, 2 \mathrm{H}$, $J=8.1), 6.58$ (t, $2 \mathrm{H}, J=2.1), 6.28(\mathrm{t}, 2 \mathrm{H}, J=2.1), 4.33(\mathrm{~s}, 2 \mathrm{H}), 3.63\left(\mathrm{q}, 2 \mathrm{H}, J_{1}=5.4, J_{2}=\right.$ 5.1), 3.33 (t, $2 \mathrm{H}, J=5.4) 2.44$ (br, $1 \mathrm{H}) ;{ }^{13} \mathrm{C}$ NMR: $\delta 160.4,160.0,155.5,137.2,128.5$, 127.2, 126.6, 122.6, 117.0, 110.5, 60.8, 52.9, 51.8; MS (ESI): $m / z 329.1\left[\mathrm{M}+\mathrm{H}^{+}\right]$. 
6-[N-Methyl(2-ethanol)amino]-4-chloro-5-pyrrol-1-yl-pyrimidine 2b: This compound was obtained by following the above procedure of 2a. $92 \%$; white solid; mp: $109-110{ }^{\circ} \mathrm{C}$; ${ }^{1} \mathrm{H}$ NMR: $\delta 8.31$ (s, 1H), 6.70 (d, 2H, $\left.J=1.8\right), 6.35$ (s, 2H), 3.81 (t, 2H, $\left.J=4.8\right), 3.60$ (t, $2 \mathrm{H}, J=4.8), 3.28$ (s, $1 \mathrm{H}), 2.61$ (s, $3 \mathrm{H}) ;{ }^{13} \mathrm{C}$ NMR: $\delta 159.9,155.3,123.5,116.8,110.2$, 109.6, 60.4, 54.6, 36.1; MS (ESI): $m / z 253.1\left[\mathrm{M}+\mathrm{H}^{+}\right]$.

General procedure for the synthesis of pyrrolo[2,1-d][1,5]pyrimidodiazepine 4 and 5,6-dihydropyrrolo[1,2-f]pteridine 5: 6-[ $N$-Benzyl(2-ethanol)amino]-4-chloro-5-pyrrol1-yl-pyrimidine 2a (164 mg, $0.5 \mathrm{mmol})$ in $6 \mathrm{~mL}$ DMSO:DCM (1:1) was treated with TEA $(0.703 \mathrm{~mL}, 5.0 \mathrm{mmol})$. A solution of $\mathrm{SO}_{3}$-Pyridine $(481 \mathrm{mg}, 3.0 \mathrm{mmol})$ in $6 \mathrm{~mL}$ DMSO:DCM (1:1) was added, and the mixture was stirred for $35 \mathrm{~min}$ at ambient temperature. The reaction solution was then diluted with DCM $(30 \mathrm{~mL})$, washed twice with $10 \%$ citric acid and brine, dried over $\mathrm{MgSO}_{4}$, and filtered to give crude aldehyde 3a in DCM. To the above solution was added the amine (aromatic amine $0.6 \mathrm{mmol}$ or aliphatic amine $3.0 \mathrm{mmol}$ ) and TFA ( $68 \mu \mathrm{L}$ ) (For aliphatic amines, additional $68 \mu \mathrm{L}$ of TFA was added after stirring $3 \mathrm{~h}$ ). The mixture was stirred for corresponding time at ambient temperature or $40{ }^{\circ} \mathrm{C}$, then washed with saturated aqueous $\mathrm{NaHCO}_{3}$, dried over $\mathrm{MgSO}_{4}$, and concentrated in vacuo. Purification by flash chromatography (Petroleum ether $/ \mathrm{EtOAc}=4: 1$ or $2: 1, \mathrm{v} / \mathrm{v}$ ) afforded the desired products.

\section{1-Chloro-5,6-dihydro-5-methyl-6-[(benzylamino)methyl]pyrrolo[1,2-f]pteridine}

5.1a: 30\%. mp: $105-106{ }^{\circ} \mathrm{C} ;{ }^{1} \mathrm{H}$ NMR: $\delta 8.18$ (s, 1H), 7.94 (t, $1 \mathrm{H}, J=1.5$ ), 7.30-7.27 (m, $2 \mathrm{H}), 7.23(\mathrm{t}, 1 \mathrm{H}, J=7.5), 7.13(\mathrm{~d}, 2 \mathrm{H}, J=7.5), 6.39$ (d, $1 \mathrm{H}, J=1.0), 6.11(\mathrm{t}, 1 \mathrm{H}, J=1.5)$, 4.70 (t, 1H, $J=4.5$ ), 3.68 (A of AB, $1 \mathrm{H}, J=13.5$ ), 3.65 (B of AB, $1 \mathrm{H}, J=13.0$ ), 3.21 (s, 3H), 2.94-2.80 (m, 2H); ${ }^{13} \mathrm{C}$ NMR: $\delta$ 154.4, 152.1, 141.5, 139.6, 128.4, 127.8, 127.0, 126.4, 119.6, 116.4, 111.3, 105.8, 58.1, 53.5, 51.3, 35.8; MS (ESI): $m / z 340.1\left[\mathrm{M}+\mathrm{H}^{+}\right]$.

\section{5-(n-Butyl)-1-chloro-5,6-dihydro-6-[(benzylamino)methyl]pyrrolo[1,2-f]pteridine}

5.2a: $32 \%$. Syrup; ${ }^{1} \mathrm{H}$ NMR: $\delta 8.14(\mathrm{~s}, 1 \mathrm{H}), 7.91\left(\mathrm{dd}, 1 \mathrm{H}, J_{1}=3.0 . J_{2}=1.5\right), 7.32-7.20$ (m, 3H), $7.15(\mathrm{~d}, 1 \mathrm{H}, J=7.2), 6.38(\mathrm{t}, 1 \mathrm{H}, J=3.3), 6.11\left(\mathrm{dd}, 1 \mathrm{H}, J_{1}=3.3 . J_{2}=1.2\right), 4.72$ $\left(\mathrm{dd}, 1 \mathrm{H}, J_{1}=7.5 . J_{2}=4.2\right), 4.30-4.21(\mathrm{~m}, 1 \mathrm{H}), 3.68(\mathrm{~s}, 2 \mathrm{H}), 3.12-3.03(\mathrm{~m}, 1 \mathrm{H}), 2.90-2.71$ $(\mathrm{m}, 2 \mathrm{H}), 1.65-1.54(\mathrm{~m}, 2 \mathrm{H}), 1.36-1.26(\mathrm{~m}, 2 \mathrm{H}), 0.91$ (t, 3H, $J=7.2) ;{ }^{13} \mathrm{C} \mathrm{NMR}: \delta 153.9$, 
152.1, 141.8, 139.6, 128.4, 127.8, 127.0, 126.4, 119.7, 116.1, 111.3, 105.7, 56.0, 53.5, 51.7, 47.3, 29.6, 20.0, 13.8; MS (ESI): $m / z 382.2\left[\mathrm{M}+\mathrm{H}^{+}\right]$.

5-Benzyl-1-chloro-5,6-dihydro-6-[(benzylamino)methyl]pyrrolo[1,2-f]pteridine 5.3a: $34 \%$. syrup. ${ }^{1} \mathrm{H}$ NMR: $\delta 8.19(\mathrm{~s}, 1 \mathrm{H}), 7.94\left(\mathrm{dd}, 1 \mathrm{H}, J_{1}=3.3, J_{2}=1.2\right), 7.34-7.20(\mathrm{~m}, 8 \mathrm{H})$, $7.12(\mathrm{~d}, 2 \mathrm{H}, J=8.1), 6.37(\mathrm{t}, 1 \mathrm{H}, J=3.3), 6.02\left(\mathrm{dd}, 1 \mathrm{H}, J_{1}=3.6, J_{2}=1.5\right), 5.57(\mathrm{~d}, 1 \mathrm{H}, J$ $=15.3), 4.68\left(\mathrm{dd}, 1 \mathrm{H}, J_{1}=6.6 . J_{2}=2.1\right), 4.35(\mathrm{~d}, 1 \mathrm{H}, J=15.3), 3.62(\mathrm{~s}, 1 \mathrm{H}), 2.85-2.72$ (m, 2H); ${ }^{13} \mathrm{C}$ NMR: $\delta 154.6,152.3,142.4,140.0,136.6,129.0,128.7,128.13,128.07$, $128.01,127.3,126.9,119.9,116.7,111.6,106.1,55.1,53.7,51.7,50.4 ;$ MS (ESI): $\mathrm{m} / \mathrm{z}$ $416.1\left[\mathrm{M}+\mathrm{H}^{+}\right]$.

\section{1-Chloro-5-cyclohexyl-5,6-dihydro-6-[(benzylamino)methyl]pyrrolo[1,2-f]pteridine}

5.4a: $35 \%$. syrup; ${ }^{1} \mathrm{H}$ NMR: $\delta 8.17(\mathrm{~s}, 1 \mathrm{H}), 7.85\left(\mathrm{dd}, 1 \mathrm{H}, J_{1}=3.3, J_{1}=1.5\right), 7.32-7.20$ (m, 3H), $7.16(\mathrm{~d}, 2 \mathrm{H}, J=8.1), 6.38(\mathrm{t}, 1 \mathrm{H}, J=3.3), 6.09\left(\mathrm{dd}, 1 \mathrm{H}, J_{1}=3.3, J_{1}=1.5\right), 4.83$ $\left(\mathrm{dd}, 1 \mathrm{H}, J_{1}=9.0 . J_{2}=3.9\right), 4.53-4.50(\mathrm{~m}, 1 \mathrm{H}), 3.69(\mathrm{~s}, 1 \mathrm{H}), 2.78-2.61(\mathrm{~m}, 2 \mathrm{H}), 1.98(\mathrm{~d}$, $1 \mathrm{H}, J=6.3), 1.83$ (br, 2H), 1.72-1.47 (m, 4H), 1.47-1.37 (m, 4H); ${ }^{13} \mathrm{C}$ NMR: $\delta 153.9$, 152.0, 142.4, 139.7, 128.3, 127.7, 127.4, 127.0, 119.6, 116.3, 111.3, 105.1, 57.1, 53.3, 53.1, 50.8, 31.7, 30.7, 25.93, 25.86, 25.4; MS (ESI): $m / z 408.0\left[\mathrm{M}+\mathrm{H}^{+}\right]$.

\section{1-Chloro-5,6-dihydro-6-[(benzylamino)methyl]-5-(iso-propyl)pyrrolo[1,2-f]pteridine}

5.5a: 42\%. mp: $62-63{ }^{\circ} \mathrm{C} .{ }^{1} \mathrm{H}$ NMR: $\delta 8.17(\mathrm{~s}, 1 \mathrm{H}), 7.86(\mathrm{~d}, 1 \mathrm{H}, J=1.5), 7.30-7.21(\mathrm{~m}$, $3 \mathrm{H}), 7.15$ (d, 2H, $J=7.0), 6.38$ (d, 1H, $J=3.5), 6.11(\mathrm{~s}, 1 \mathrm{H}), 4.88-4.83(\mathrm{~m}, 2 \mathrm{H}), 3.68$ (s, $2 \mathrm{H}), 2.80-2.64(\mathrm{~m}, 2 \mathrm{H}), 1.32(\mathrm{~d}, 3 \mathrm{H}, J=7.0), 1.28(\mathrm{~d}, 3 \mathrm{H}, J=6.5) ;{ }^{13} \mathrm{C} \mathrm{NMR}: \delta 153.0$, 151.0, 141.4, 138.6, 127.4, 126.8, 126.3, 126.1, 118.6, 115.4, 110.4, 104.3, 52.3, 52.1, 49.6, 48.1, 20.3, 19.4; MS (ESI): $m / z 368.0\left[\mathrm{M}+\mathrm{H}^{+}\right]$.

\section{1-Chloro-5,6-dihydro-6-[(benzylamino)methyl]-5-(2-methoxylphenyl)pyrrolo[1,2-f]}

pteridine 5.6a: $61 \%$. syrup. ${ }^{1} \mathrm{H}$ NMR: $\delta 8.05(\mathrm{~s}, 1 \mathrm{H}), 8.02\left(\mathrm{dd}, 1 \mathrm{H}, J_{1}=3.0 . J_{2}=1.2\right)$, 7.37 (t, $1 \mathrm{H}, J=7.5), 7.28-7.22(\mathrm{~m}, 4 \mathrm{H}), 7.10(\mathrm{~d}, 2 \mathrm{H}, J=6.3), 7.02$ (d, 2H, $J=8.1), 6.43$ (t, $1 \mathrm{H}, J=3.3), 6.12(\mathrm{~d}, 1 \mathrm{H}, J=2.4), 5.00(\mathrm{t}, 1 \mathrm{H}, J=4.8), 3.74(\mathrm{~s}, 3 \mathrm{H}), 3.62(\mathrm{~s}, 2 \mathrm{H})$, $2.88(\mathrm{~d}, 2 \mathrm{H}, J=5.4) ;{ }^{13} \mathrm{C}$ NMR: $\delta 151.83,151.79,142.2,139.9,131.1,129.5,128.3$, $127.8,126.9,126.8,121.0,119.64,119.61,116.0,112.4,111.4,105.8,57.0,55.5,53.5$, 52.1; MS (ESI): $m / z 432.0\left[\mathrm{M}+\mathrm{H}^{+}\right]$. 


\section{1-Chloro-5,6-dihydro-6-[(benzylamino)methyl]-5-(4-methoxylphenyl)pyrrolo[1,2-f]}

pteridine 5.7a: $40 \%$. mp: $111-113{ }^{\circ} \mathrm{C} .{ }^{1} \mathrm{H}$ NMR: $\delta 8.10(\mathrm{~s}, 1 \mathrm{H}), 7.99\left(\mathrm{dd}, 1 \mathrm{H}, J_{1}=3.0\right.$. $\left.J_{2}=1.5\right), 7.30-7.23(\mathrm{~m}, 3 \mathrm{H}), 7.17-7.10(\mathrm{~m}, 4 \mathrm{H}), 6.96-6.92(\mathrm{~m}, 2 \mathrm{H}), 6.44(\mathrm{t}, 1 \mathrm{H}, J=3.3)$, $6.15(\mathrm{~d}, 1 \mathrm{H}, J=2.1), 5.01\left(\mathrm{dd}, 1 \mathrm{H}, J_{1}=6.6 . J_{2}=3.6\right), 3.83(\mathrm{~s}, 3 \mathrm{H}), 3.67$ (A of AB, $1 \mathrm{H}, J$ $=13.2), 3.61(\mathrm{~B}$ of $\mathrm{AB}, 1 \mathrm{H}, J=13.2), 3.01-2.85(\mathrm{~m}, 2 \mathrm{H}) ;{ }^{13} \mathrm{C}$ NMR: $\delta 158.6,154.3$, 152.0, 142.6, 139.7, 134.0, 128.8, 128.4, 127.9, 127.0, 126.6, 119.8, 116.4, 114.7, 111.6, 106.0, 59.3, 55.4, 53.5, 52.0; MS (ESI): $m / z 432.1\left[\mathrm{M}+\mathrm{H}^{+}\right]$.

\section{7-(2-Methylphenylamino)-5-benzyl-1-chloro-6,7-dihydropyrrolo[2,1-d][1,5]}

benzodiazepine 4.8a: $86 \%$. mp: $127-128{ }^{\circ} \mathrm{C} .{ }^{1} \mathrm{H}$ NMR: $\delta 8.35(\mathrm{~s}, 1 \mathrm{H}), 7.29-7.21(\mathrm{~m}$, $5 \mathrm{H}), 7.02-6.98(\mathrm{~m}, 2 \mathrm{H}), 6.91(\mathrm{t}, 1 \mathrm{H}, J=6.9), 6.65(\mathrm{t}, 1 \mathrm{H}, J=7.2), 6.37(\mathrm{~d}, 1 \mathrm{H}, J=$ 8.1), $6.27(\mathrm{t}, 1 \mathrm{H}, J=3.3), 6.16(\mathrm{t}, 1 \mathrm{H}, J=1.5), 4.90(\mathrm{br}, 2 \mathrm{H}),$,4.79 (t, 1H, $J=5.1$ ), 3.83-3.63 (m, 3H), 1.99 (s, 3H); ${ }^{13} \mathrm{C}$ NMR: $\delta$ 157.1, 153.7, 153.4, 143.4, 136.6, 133.6, 130.3, 128.8, 127.9, 127.7, 127.1, 124.3, 122.7, 118.3, 116.8, 110.8, 109.1, 105.4, 57.7, 55.0, 48.9, 17.3; MS (ESI): $m / z 416.1\left[\mathrm{M}+\mathrm{H}^{+}\right]$.

\section{1-Chloro-5,6-dihydro-6-[(benzylamino)methyl]-5-(3-methylphenyl)pyrrolo[1,2-f]}

pteridine 5.9a: $31 \%$. mp: $103-105{ }^{\circ} \mathrm{C} .{ }^{1} \mathrm{H}$ NMR: $\delta 8.11(\mathrm{~s}, 1 \mathrm{H}), 7.98\left(\mathrm{dd}, 1 \mathrm{H}, J_{1}=3.0\right.$. $\left.J_{2}=1.5\right), 7.33-7.22(\mathrm{~m}, 4 \mathrm{H}), 7.14-7.05(\mathrm{~m}, 5 \mathrm{H}), 6.43(\mathrm{t}, 1 \mathrm{H}, J=3.3), 6.14\left(\mathrm{dd}, 1 \mathrm{H}, J_{1}=\right.$ 3.3. $\left.J_{2}=0.9\right), 5.04\left(\mathrm{dd}, 1 \mathrm{H}, J_{1}=6.6 . J_{2}=3.6\right), 3.68(\mathrm{~A}$ of $\mathrm{AB}, 1 \mathrm{H}, J=13.5), 3.62$ (B of $\mathrm{AB}, 1 \mathrm{H}, J=13.5), 3.01-2.85(\mathrm{~m}, 2 \mathrm{H}), 2.35(\mathrm{~s}, 3 \mathrm{H}) ;{ }^{13} \mathrm{C}$ NMR: $\delta 153.9,152.0,142.8$, $141.4,139.6,139.5,129.2,128.34,128.25,127.9,127.5,127.0,126.6,124.1,119.7$, 116.6, 111.6, 106.0, 58.9, 53.4, 51.9, 21.4; MS (ESI): $m / z 416.2\left[\mathrm{M}+\mathrm{H}^{+}\right]$.

7-(4-Methylphenylamino)-5-benzyl-1-chloro-6,7-dihydropyrrolo[2,1-d][1,5]

pyrimidodiazepine 4.10a: $26 \%$. mp: $175-176{ }^{\circ} \mathrm{C} .{ }^{1} \mathrm{H}$ NMR: $\delta 8.34(\mathrm{~s}, 1 \mathrm{H}), 7.31-7.22$ $(\mathrm{m}, 5 \mathrm{H}), 6.98\left(\mathrm{dd}, 1 \mathrm{H}, J_{1}=3.0 . J_{2}=1.5\right), 6.88(\mathrm{~d}, 2 \mathrm{H}, J=7.5), 6.40(\mathrm{~d}, 2 \mathrm{H}, J=8.4), 6.25$ (t, $1 \mathrm{H}, J=3.3$ ), $6.12\left(\mathrm{dd}, 1 \mathrm{H}, J_{1}=3.6 . J_{2}=0.9\right), 4.96(\mathrm{br}, 1 \mathrm{H}), 4.83(\mathrm{br}, 1 \mathrm{H}), 4.69$ (br, $1 \mathrm{H}), 3.76\left(\mathrm{dd}, 1 \mathrm{H}, J_{1}=12.0 . J_{2}=2.1\right), 3.63(\mathrm{br}, 2 \mathrm{H}), 2.20(\mathrm{~s}, 3 \mathrm{H}) ;{ }^{13} \mathrm{C} \mathrm{NMR}: \delta 156.9$, 153.7, 143.4, 136.5, 133.9, 129.7, 128.7, 128.0, 127.8, 127.7, 124.1, 114.0, 109.71, 109.68, 109.0, 105.2, 58.3, 55.0, 49.6, 20.3; MS (ESI): $m / z 416.1\left[\mathrm{M}+\mathrm{H}^{+}\right]$. 


\section{1-Chloro-5,6-dihydro-6-[(benzylamino)methyl]-5-(4-methylphenyl)pyrrolo[1,2-f]}

pteridine 5.10a: $58 \%$. mp: $113-114{ }^{\circ} \mathrm{C} .{ }^{1} \mathrm{H}$ NMR: $\delta 8.10(\mathrm{~s}, 1 \mathrm{H}), 7.98\left(\mathrm{dd}, 1 \mathrm{H}, J_{1}=3.3\right.$. $\left.J_{2}=1.5\right), 7.31-7.21(\mathrm{~m}, 5 \mathrm{H}), 7.15-7.10(\mathrm{~m}, 4 \mathrm{H}), 6.43(\mathrm{t}, 1 \mathrm{H}, J=3.3), 6.13\left(\mathrm{dd}, 1 \mathrm{H}, J_{1}=\right.$ 3.6. $J_{2}=1.5$ ), $5.02\left(\mathrm{dd}, 1 \mathrm{H}, J_{1}=6.6 . J_{2}=4.2\right.$ ), 3.67 (A of AB, $1 \mathrm{H}, J=13.2$ ), 3.61 (B of $\mathrm{AB}, 1 \mathrm{H}, J=13.5), 3.01-2.86\left(\mathrm{~m}, 2 \mathrm{H}\right.$ ), $2.38(\mathrm{~s}, 3 \mathrm{H}) ;{ }^{13} \mathrm{C} \mathrm{NMR}: \delta 154.0,151.8,142.6$, 139.6, 138.6, 137.3, 130.0, 128.3, 127.8, 127.0, 126.9, 126.5, 119.6, 116.4, 111.5, 105.9, 59.0, 53.3, 51.9, 21.0; MS (ESI): $m / z 416.0\left[\mathrm{M}+\mathrm{H}^{+}\right]$.

\section{1-Chloro-5,6-dihydro-6-[(benzylamino)methyl]-5-phenylpyrrolo[1,2-f]pteridine}

5.11a: 55\%. mp: 91-92 ${ }^{\circ} \mathrm{C} .{ }^{1} \mathrm{H}$ NMR: $\delta 8.10(\mathrm{~s}, 1 \mathrm{H}), 7.97(\mathrm{~d}, 1 \mathrm{H}, \quad J=1.5), 7.41(\mathrm{t}, 2 \mathrm{H}, J$ $=7.5), 7.33-7.19(\mathrm{~m}, 6 \mathrm{H}), 7.10(\mathrm{~d}, 2 \mathrm{H}, J=6.9), 6.42(\mathrm{t}, 1 \mathrm{H}, J=3.3), 6.13(\mathrm{~d}, 1 \mathrm{H}, J=$ 2.7), 5.05 (dd, $1 \mathrm{H}, J_{1}=6.3 . J_{2}=3.6$ ), 3.66 (A of AB, $1 \mathrm{H}, J=13.5$ ), 3.60 (B of AB, $1 \mathrm{H}, J$ $=13.5), 3.00-2.85(\mathrm{~m}, 2 \mathrm{H}) ;{ }^{13} \mathrm{C}$ NMR: $\delta 153.8,151.8,147.2,142.9,141.4,139.6,129.4$, $128.3,127.8,127.3,127.0,126.6,119.7,116.7,111.5,106.0,58.9,53.4,51.9 ; M S$ (ESI): $\mathrm{m} / \mathrm{z} 402.2\left[\mathrm{M}+\mathrm{H}^{+}\right]$.

\section{7-(2-Chlorophenylamino)-5-benzyl-1-chloro-6,7-dihydropyrrolo[2,1-d][1,5]}

pyrimidodiazepine 4.12a: $64 \%$. mp: $120-121{ }^{\circ} \mathrm{C} .{ }^{1} \mathrm{H}$ NMR: $\delta 8.36$ (s, $\left.1 \mathrm{H}\right), 7.31-7.27$ (m, 5H), 7.21 (d, 1H, $J=7.5), 6.99$ (s, 1H), 6.93 (t, 1H, $J=7.5), 6.63(\mathrm{t}, 1 \mathrm{H}, J=7.5$ ), $6.45(\mathrm{br}, 1 \mathrm{H}), 6.27(\mathrm{~d}, 1 \mathrm{H}, J=2.5), 6.19(\mathrm{~s}, 1 \mathrm{H}), 4.92(\mathrm{br}, 2 \mathrm{H}), 4.78(\mathrm{t}, 1 \mathrm{H}, J=6.5), 4.51$ (s, 1H), 3.81-3.72 (m, 2H,); ${ }^{13} \mathrm{C}$ NMR: $\delta$ 156.9, 153.6, 153.4, 141.3, 136.4, 132.6, 129.1, $128.7,127.8,127.7,127.6,124.3,119.7,118.5,116.5,112.3,109.0,105.8,57.6,54.9$, 48.8; MS (ESI): $m / z 436.0\left[\mathrm{M}+\mathrm{H}^{+}\right]$.

\section{1-Chloro-5,6-dihydro-6-[(benzylamino)methyl]-5-(3-chlorophenyl)pyrrolo[1,2-f]}

pteridine 5.13a: $38 \%$. mp: $108-109{ }^{\circ} \mathrm{C} .{ }^{1} \mathrm{H}$ NMR: $\delta 8.15$ (s, $1 \mathrm{H}$ ), 7.97 (dd, $1 \mathrm{H}, J_{1}=3.3$. $\left.J_{2}=1.5\right), 7.36-7.23(\mathrm{~m}, 6 \mathrm{H}), 7.19-7.11(\mathrm{~m}, 3 \mathrm{H}), 6.44(\mathrm{t}, 1 \mathrm{H}, J=3.3), 6.14\left(\mathrm{dd}, 1 \mathrm{H}, J_{1}=\right.$ 3.3. $J_{2}=1.2$ ), 5.05 (dd, $1 \mathrm{H}, J_{1}=6.0 . J_{2}=3.9$ ), 3.69 (A of AB, $\left.1 \mathrm{H}, J=13.5\right), 3.63$ (B of $\mathrm{AB}, 1 \mathrm{H}, J=13.2), 3.00-2.84(\mathrm{~m}, 2 \mathrm{H}) ;{ }^{13} \mathrm{C}$ NMR: $\delta 153.5,151.8,143.3,142.7,139.5$, 134.7, 130.1, 128.4, 127.8, 127.2, 127.1, 126.9, 126.5, 124.9, 119.7, 117.0, 111.6, 106.1, 58.8, 53.4, 51.9; MS (ESI): $m / z 436.1\left[\mathrm{M}+\mathrm{H}^{+}\right]$.

1-Chloro-5,6-dihydro-6-[(benzylamino)methyl]-5-(4-chlorophenyl)pyrrolo[1,2-f]

pteridine 5.14a: $62 \%$. mp: $139-140{ }^{\circ} \mathrm{C} .{ }^{1} \mathrm{H}$ NMR: $\delta 8.12(\mathrm{~s}, 1 \mathrm{H}), 8.00-7.98(\mathrm{~m}, 1 \mathrm{H})$, 
7.38-7.34 (m, 2H), 7.31-7.24 (m, 3H), 7.20-7.17 (m, 2H), 7.12-7.09 (m, 2H), $6.45(\mathrm{t}, 1 \mathrm{H}$ , $J=3.3$ ), 6.14 (d, $1 \mathrm{H}, J=3.0), 5.02\left(\mathrm{dd}, 1 \mathrm{H}, J_{1}=6.0 . J_{2}=3.9\right), 3.68$ (A of AB, $1 \mathrm{H}, J=$ 13.2), 3.60 (B of $\mathrm{AB}, 1 \mathrm{H}, J=13.5), 2.98-2.83(\mathrm{~m}, 2 \mathrm{H}) ;{ }^{13} \mathrm{C}$ NMR: $\delta$ 153.7, 151.7, 143.1, 139.9, 139.6, 132.7, 129.5, 128.3, 128.3, 127.9, 127.0, 126.4, 119.7, 116.9, 111.6, 106.1, 58.9, 53.3, 51.8; MS (ESI): $\mathrm{m} / z 436.0\left[\mathrm{M}+\mathrm{H}^{+}\right]$.

\section{1-Chloro-5,6-dihydro-6-[(benzylamino)methyl]-5-(4-florophenyl)pyrrolo[1,2-f]}

pteridine 5.15a: $50 \%$. mp: $136-138{ }^{\circ} \mathrm{C} .{ }^{1} \mathrm{H}$ NMR: $\delta 8.10(\mathrm{~s}, 1 \mathrm{H}), 8.00\left(\mathrm{dd}, 1 \mathrm{H}, J_{1}=3.0\right.$. $\left.J_{2}=1.5\right), 7.30-7.18(\mathrm{~m}, 5 \mathrm{H}), 7.12-7.06(\mathrm{~m}, 4 \mathrm{H}), 6.45(\mathrm{t}, 1 \mathrm{H}, J=3.3), 6.14\left(\mathrm{dd}, 1 \mathrm{H}, J_{1}=\right.$ 3.6. $\left.J_{2}=1.5\right), 5.00\left(\mathrm{dd}, 1 \mathrm{H}, J_{1}=6.0 . J_{2}=3.9\right), 3.68(\mathrm{~A}$ of $\mathrm{AB}, 1 \mathrm{H}, J=13.5), 3.60$ (B of $\mathrm{AB}, 1 \mathrm{H}, J=13.2), 2.99-2.84(\mathrm{~m}, 2 \mathrm{H}) ;{ }^{13} \mathrm{C}$ NMR: $\delta 162.9,159.6,154.0,151.7,142.8$, 139.6, 137.2, 129.4, 129.2, 128.3, 128.0, 127.9, 127.0, 126.4, 119.7, 116.4, 116.1, 111.6, 106.0, 59.2, 53.4, 51.8; MS (ESI): $m / z 420.1\left[\mathrm{M}+\mathrm{H}^{+}\right]$.

\section{7-(2-Nitrophenylamino)-5-benzyl-1-chloro-6,7-dihydropyrrolo[2,1-d][1,5]}

pyrimidodiazepine 4.16a: 32\%. mp: $167-168{ }^{\circ} \mathrm{C} .{ }^{1} \mathrm{H}$ NMR: $\delta 8.39$ (s, 1H), 8.27 (br, $1 \mathrm{H}), 8.13(\mathrm{~d}, 1 \mathrm{H}, J=8.4), 7.31-7.20(\mathrm{~m}, 5 \mathrm{H}), 7.00(\mathrm{~s}, 1 \mathrm{H}), 6.66(\mathrm{t}, 1 \mathrm{H}, J=7.5), 6.58(\mathrm{br}$, $1 \mathrm{H}), 6.28-6.26(\mathrm{~m}, 2 \mathrm{H}), 5.04(\mathrm{br}, 2 \mathrm{H}), 4.91(\mathrm{~s}, 1 \mathrm{H}), 3.79(\mathrm{~s}, 2 \mathrm{H}) ;{ }^{13} \mathrm{C} \mathrm{NMR}: \delta 156.8$, $153.89,153.86,142.9,136.4,136.1,132.8,131.7,128.8,128.1,127.9,126.8,124.6$, 116.6, 116.3, 114.1, 109.3, 106.2, 57.4, 55.0, 48.3; MS (ESI): $m / z 447.0\left[\mathrm{M}+\mathrm{H}^{+}\right]$.

\section{1-Chloro-5,6-dihydro-6-[(benzylamino)methyl]-5-(4-nitrophenyl)pyrrolo[1,2-f]}

pteridine 5.17a: $43 \%$. mp: $186-188{ }^{\circ} \mathrm{C} .{ }^{1} \mathrm{H}$ NMR: $\delta 8.25$ (s, $\left.1 \mathrm{H}\right), 8.22(\mathrm{~d}, 2 \mathrm{H}, J=9.0$ ), 7.95 (d, $1 \mathrm{H}, J=1.8$ ), 7.47 (d, 2H, $J=9.3$ ), 7.34-7.26 (m, 3H), 7.16 (d, 2H, $J=7.5$ ), 6.45 (t, $2 \mathrm{H}, J=3.6), 6.18$ (d, $1 \mathrm{H}, J=2.7), 5.17$ (t, $1 \mathrm{H}, J=6.6), 3.70$ (d, $1 \mathrm{H}, J=3.6), 3.02-2.85$ (m, 2H); ${ }^{13} \mathrm{C}$ NMR: $\delta 152.3,151.4,146.2,144.8,130.1,129.8,129.6,129.4,129.3$, $124.9,123.4,122.8,121.4,118.1,112.5,109.3,53.4,51.5,46.4$; MS (ESI): $m / z 447.0$ $\left[\mathrm{M}+\mathrm{H}^{+}\right]$.

\section{1-Chloro-5,6-dihydro-5-[benzylhydroxyl]-6-[(benzylamino)methyl]pyrrolo[1,2-f]}

pteridine 5.18a: $21 \%$. mp: $90-91{ }^{\circ} \mathrm{C} .{ }^{1} \mathrm{H}$ NMR: $\delta 8.37$ (s, $\left.1 \mathrm{H}\right), 7.92\left(\mathrm{dd}, 1 \mathrm{H}, J_{1}=3.3 . J_{2}\right.$ $=1.2), 7.48-7.45(\mathrm{~m}, 2 \mathrm{H}), 7.38-7.36(\mathrm{~m}, 3 \mathrm{H}), 7.27-7.16(\mathrm{~m}, 3 \mathrm{H}), 7.06(\mathrm{~d}, 2 \mathrm{H}, J=6.3)$, 6.32 (t, $1 \mathrm{H}, J=3.3$ ), 6.10 (d, $1 \mathrm{H}, J=2.4$ ), 5.12 (d, $1 \mathrm{H}, J=10.8), 4.99$ (d, $1 \mathrm{H}, J=10.8$ ), 4.77 (t, $1 \mathrm{H}, J=4.2$ ), 3.61 (A of AB, 1H, $J=13.5$ ), 3.58 (B of AB, $1 \mathrm{H}, J=13.5$ ), 3.13- 
$3.00(\mathrm{~m}, 1 \mathrm{H}) ;{ }^{13} \mathrm{C}$ NMR: $\delta 156.2,152.5,142.0,139.5,135.1,129.4,128.8,128.6,128.3$, 127.7, 127.0, 126.9, 119.8, 116.8, 111.0, 107.1, 77.2, 59.1, 53.6, 50.2; MS (ESI): $\mathrm{m} / \mathrm{z}$ $432.1\left[\mathrm{M}+\mathrm{H}^{+}\right]$.

\section{1-Chloro-5,6-dihydro-6-[(methylamino)methyl]-5-(4-methylphenyl)pyrrolo[1,2-f]}

pteridine 5.10b: $51 \%$. mp: $122-124{ }^{\circ} \mathrm{C} .{ }^{1} \mathrm{H}$ NMR: $\delta 8.11(\mathrm{~s}, 1 \mathrm{H}), 7.98-7.97(\mathrm{~m}, 1 \mathrm{H})$, 7.28 (d, 1H, $J=8.7), 7.24$ (d, 1H, $J=8.4$ ), 6.44 (t, $1 \mathrm{H}, J=3.3), 6.15$ (d, $1 \mathrm{H}, J=3.0$ ), $5.02\left(\mathrm{dd}, 1 \mathrm{H}, J_{1}=7.1 . J_{2}=4.2\right), 2.94-2.77(\mathrm{~m}, 2 \mathrm{H}), 2.39(\mathrm{~s}, 3 \mathrm{H}), 2.33(\mathrm{~s}, 3 \mathrm{H}) ;{ }^{13} \mathrm{C} \mathrm{NMR}$ : \& 154.0, 152.4, 143.3, 139.1, 137.8, 130.5, 127.2, 126.6, 120.1, 116.5, 111.9, 106.5, 59.0, 54.7, 36.6, 21.4; MS (ESI): $\mathrm{m} / z 340.1\left[\mathrm{M}+\mathrm{H}^{+}\right]$.

\section{1-Chloro-5,6-dihydro-6-[(methylamino)methyl]-5-(4-chlorophenyl)pyrrolo[1,2-f]}

pteridine 5.14b: $36 \%$. mp: $86-87{ }^{\circ} \mathrm{C} .{ }^{1} \mathrm{H}$ NMR: $\delta 8.13$ (s, $\left.1 \mathrm{H}\right), 7.97(\mathrm{~d}, 1 \mathrm{H}, J=1.8$ ), 7.43 (d, 1H, $J=8.7), 7.33$ (d, 1H, $J=8.7), 6.44$ (t, 1H, $J=3.3), 6.16$ (d, 1H, $J=2.7$ ), $5.03\left(\mathrm{dd}, 1 \mathrm{H}, J_{1}=7.8 . J_{2}=4.5\right), 2.88-2.78(\mathrm{~m}, 2 \mathrm{H}), 2.33(\mathrm{~s}, 3 \mathrm{H}) ;{ }^{13} \mathrm{C} \mathrm{NMR}: \delta 153.7$, 152.2, 143.7, 140.3, 133.1, 129.9, 128.5, 126.5, 120.2, 117.0, 112.0, 106.7, 58.9, 54.7, 36.6; MS (ESI): $m / z 360.0\left[\mathrm{M}+\mathrm{H}^{+}\right]$.

5-Benzyl-1-chloro-6,7-dihydro-7-hydroxylpyrrolo[2,1-d][1,5]pyrimido- diazepine 6a: 6-[N-Benzyl(2-ethanol)amino]-4-chloro-5-pyrrol-1-yl-pyrimidine 2a (164 mg, $0.5 \mathrm{mmol})$ in $6 \mathrm{~mL}$ DMSO:DCM (1:1) was treated with TEA $(0.703 \mathrm{~mL}, 5.0 \mathrm{mmol})$. A solution of $\mathrm{SO}_{3}$-Pyridine (481 mg, $3.0 \mathrm{mmol}$ ) in $6 \mathrm{~mL}$ DMSO:DCM (1:1) was added, and the mixture was stirred for $35 \mathrm{~min}$ at ambient temperature. The reaction solution was diluted with DCM (30 mL), washed twice with $10 \%$ citric acid and brine, dried over $\mathrm{MgSO}_{4}$, filtered, and concentrated in vacuo to give crude product 3a. Crude product 3a was dissolved in DCM $(0.5 \mathrm{~mL})$ and mixed with silica gel $(0.6 \mathrm{~g})$. The mixture was stirred overnight in air. Purification by flash chromatography (Petroleum ether $/ \operatorname{EtOAc}=4: 1, \mathrm{v} / \mathrm{v}$ ) afforded $74 \mathrm{mg}$ (45\%) of $\mathbf{6 a}$ as a white solid. mp: $171-173{ }^{\circ} \mathrm{C} .{ }^{1} \mathrm{H}$ NMR: $\delta 8.26(\mathrm{~s}, 1 \mathrm{H})$, 7.36-7.24 (m, 5H), $7.01\left(\mathrm{dd}, 1 \mathrm{H}, J_{1}=3.0 . J_{2}=1.8\right), 6.23(\mathrm{t}, 1 \mathrm{H}, J=3.0), 6.16\left(\mathrm{dd}, 1 \mathrm{H}, J_{1}\right.$ $\left.=3.6 . J_{2}=1.8\right), 5.10(\mathrm{~d}, 1 \mathrm{H}, J=15.3), 5.00\left(\mathrm{dd}, 1 \mathrm{H}, J_{1}=5.1 . J_{2}=2.1\right), 4.69(\mathrm{~d}, 1 \mathrm{H}, J=$ 15.3), 3.81-3.67 (m, 2H); ${ }^{13} \mathrm{C}$ NMR: $\delta 157.8,153.1,153.0,152.0,136.4,133.1,128.6$, 127.5, 127.3, 124.8, 108.7, 106.9, 64.3, 59.2, 55.0; MS (ESI): $m / z 327.2\left[\mathrm{M}+\mathrm{H}^{+}\right]$. 
Figure Legends:

Fig. S-1: LC-MS-ELSD of 6-[ $N$-benzyl(2-ethanol)amino]-4-chloro-5-pyrrol-1-ylpyrimidine $2 \mathrm{a}$.

Fig. S-2: ${ }^{1} \mathrm{H}$ Spectra of 6-[N-benzyl(2-ethanol)amino]-4-chloro-5-pyrrol-1-ylpyrimidine 2a.

Fig. S-3: ${ }^{13} \mathrm{C}$ Spectra of 6-[N-benzyl(2-ethanol)amino]-4-chloro-5-pyrrol-1-ylpyrimidine 2a.

Fig. S-4: LC-MS-ELSD of 6-[ $N$-methyl(2-ethanol)amino]-4-chloro-5-pyrrol-1-ylpyrimidine $2 b$.

Fig. S-5: ${ }^{1} \mathrm{H}$ Spectra of 6-[ $N$-methyl(2-ethanol)amino]-4-chloro-5-pyrrol-1-ylpyrimidine $\mathbf{2 b}$.

Fig. S-6: ${ }^{13} \mathrm{C}$ Spectra of 6-[ $N$-methyl(2-ethanol)amino]-4-chloro-5-pyrrol-1-ylpyrimidine $2 \mathbf{b}$.

Fig. S-7: LC-MS-ELSD of 1-chloro-5,6-dihydro-5-methyl-6-[(benzylamino)methyl] pyrrolo[1,2-f]pteridine 5.1a.

Fig. S-8: ${ }^{1} \mathrm{H}$ Spectra of 1-chloro-5,6-dihydro-5-methyl-6-[(benzylamino)methyl] pyrrolo[1,2-f]pteridine 5.1a.

Fig. S-9: ${ }^{13} \mathrm{C}$ Spectra of 1-chloro-5,6-dihydro-5-methyl-6-[(benzylamino)methyl] pyrrolo[1,2-f]pteridine 5.1a.

Fig. S-10: LC-MS-ELSD of 5-(n-butyl)-1-chloro-5,6-dihydro-6-[(benzylamino) methyl]pyrrolo[1,2-f]pteridine 5.2a.

Fig. S-11: ${ }^{1} \mathrm{H} \quad$ Spectra of 5-(n-butyl)-1-chloro-5,6-dihydro-6-[(benzylamino) methyl]pyrrolo[1,2-f]pteridine 5.2a..

Fig. S-12: ${ }^{13} \mathrm{C}$ Spectra of 5-(n-butyl)-1-chloro-5,6-dihydro-6-[(benzylamino) methyl]pyrrolo[1,2-f]pteridine 5.2a..

Fig. S-13: LC-MS-ELSD of 5-benzyl-1-chloro-5,6-dihydro-6-[(benzylamino) methyl]pyrrolo[1,2-f]pteridine 5.3a.

Fig. S-14: ${ }^{1} \mathrm{H}$ Spectra of 5-benzyl-1-chloro-5,6-dihydro-6-[(benzylamino)methyl] pyrrolo[1,2-f]pteridine 5.3a.

Fig. S-15: ${ }^{13} \mathrm{C}$ Spectra of 5-benzyl-1-chloro-5,6-dihydro-6-[(benzylamino)methyl] pyrrolo[1,2-f]pteridine 5.3a. 
Fig. S-16: LC-MS-ELSD of 1-chloro-5-cyclohexyl-5,6-dihydro-6-[(benzylamino) methyl]pyrrolo[1,2-f]pteridine 5.4a.

Fig. S-17: ${ }^{1} \mathrm{H}$ Spectra of 1-chloro-5-cyclohexyl-5,6-dihydro-6-[(benzylamino) methyl]pyrrolo[1,2-f]pteridine 5.4a.

Fig. S-18: ${ }^{13} \mathrm{C}$ Spectra of 1-chloro-5-cyclohexyl-5,6-dihydro-6-[(benzylamino) methyl]pyrrolo[1,2-f]pteridine 5.4a.

Fig. S-19: LC-MS-ELSD of 1-chloro-5,6-dihydro-6-[(benzylamino)methyl]-5-(isopropyl)pyrrolo[1,2-f]pteridine 5.5a.

Fig. S-20: ${ }^{1} \mathrm{H}$ Spectra of 1-chloro-5,6-dihydro-6-[(benzylamino)methyl]-5-(isopropyl)pyrrolo[1,2-f]pteridine 5.5a.

Fig. S-21: ${ }^{13} \mathrm{C}$ Spectra of 1-chloro-5,6-dihydro-6-[(benzylamino)methyl]-5-(isopropyl)pyrrolo[1,2-f]pteridine 5.5a.

Fig. S-22: LC-MS-ELSD of 1-chloro-5,6-dihydro-6-[(benzylamino)methyl]-5-(2methoxylphenyl)pyrrolo[1,2-f]pteridine 5.6a.

Fig. S-23: $\quad{ }^{1} \mathrm{H}$ Spectra of 1-chloro-5,6-dihydro-6-[(benzylamino)methyl]-5-(2methoxylphenyl)pyrrolo[1,2-f]pteridine 5.6a.

Fig. S-24: ${ }^{13} \mathrm{C}$ Spectra of 1-chloro-5,6-dihydro-6-[(benzylamino)methyl]-5-(2methoxylphenyl)pyrrolo[1,2-f]pteridine 5.6a.

Fig. S-25: LC-MS-ELSD of 1-chloro-5,6-dihydro-6-[(benzylamino)methyl]-5-(4methoxylphenyl)pyrrolo[1,2-f]pteridine 5.7a.

Fig. S-26: ${ }^{1} \mathrm{H}$ Spectra of 1-chloro-5,6-dihydro-6-[(benzylamino)methyl]-5-(4methoxylphenyl)pyrrolo[1,2-f]pteridine 5.7a.

Fig. S-27: ${ }^{13} \mathrm{C}$ Spectra of 1-chloro-5,6-dihydro-6-[(benzylamino)methyl]-5-(4methoxylphenyl)pyrrolo[1,2-f]pteridine 5.7a.

Fig. S-28: LC-MS-ELSD of 7-(2-methylphenylamino)-5-benzyl-1-chloro-6,7dihydropyrrolo[2,1-d][1,5]benzodiazepine 4.8a.

Fig. S-29: $\quad{ }^{1} \mathrm{H} \quad$ Spectra of 7-(2-methylphenylamino)-5-benzyl-1-chloro-6,7dihydropyrrolo[2,1-d][1,5]benzodiazepine 4.8a.

Fig. $\quad$ S-30: ${ }^{13} \mathrm{C}$ Spectra of 7-(2-methylphenylamino)-5-benzyl-1-chloro-6,7dihydropyrrolo[2,1-d][1,5]benzodiazepine 4.8a. 
Fig. S-31: LC-MS-ELSD of 1-chloro-5,6-dihydro-6-[(benzylamino)methyl]-5-(3methylphenyl)pyrrolo[1,2-f]pteridine 5.9a.

Fig. S-32: $\quad{ }^{1} \mathrm{H}$ Spectra of 1-chloro-5,6-dihydro-6-[(benzylamino)methyl]-5-(3methylphenyl)pyrrolo[1,2-f]pteridine 5.9a.

Fig.S-33: $\quad{ }^{13} \mathrm{C}$ Spectra of 1-chloro-5,6-dihydro-6-[(benzylamino)methyl]-5-(3methylphenyl)pyrrolo[1,2-f]pteridine 5.9a.

Fig. S-34: LC-MS-ELSD of 7-(4-methylphenylamino)-5-benzyl-1-chloro-6,7-dihydro pyrrolo[2,1-d][1,5]pyrimidodiazepine 4.10a

Fig. S-35: ${ }^{1} \mathrm{H}$ Spectra of 7-(4-methylphenylamino)-5-benzyl-1-chloro-6,7-dihydro pyrrolo[2,1-d][1,5]pyrimidodiazepine 4.10a.

Fig. S-36: ${ }^{13} \mathrm{C}$ Spectra of 7-(4-methylphenylamino)-5-benzyl-1-chloro-6,7-dihydro pyrrolo[2,1-d][1,5]pyrimidodiazepine 4.10a.

Fig. S-37: LC-MS-ELSD of 1-chloro-5,6-dihydro-6-[(benzylamino)methyl]-5-(4methylphenyl)pyrrolo[1,2-f]pteridine 5.10a

Fig. S-38: ${ }^{1} \mathrm{H}$ Spectra of 1-chloro-5,6-dihydro-6-[(benzylamino)methyl]-5-(4methylphenyl)pyrrolo[1,2-f]pteridine 5.10a.

Fig. S-39: ${ }^{13} \mathrm{C}$ Spectra of 1-chloro-5,6-dihydro-6-[(benzylamino)methyl]-5-(4methylphenyl)pyrrolo[1,2-f]pteridine 5.10a.

Fig. S-40: LC-MS-ELSD of 1-chloro-5,6-dihydro-6-[(benzylamino)methyl]-5-phenyl pyrrolo[1,2-f]pteridine 5.11a.

Fig. S-41: ${ }^{1} \mathrm{H}$ Spectra of 1-chloro-5,6-dihydro-6-[(benzylamino)methyl]-5-phenyl pyrrolo[1,2-f]pteridine 5.11a.

Fig. S-42: ${ }^{13} \mathrm{C}$ Spectra of 1-chloro-5,6-dihydro-6-[(benzylamino)methyl]-5-phenyl pyrrolo[1,2-f]pteridine 5.11a.

Fig. S-43: LC-MS-ELSD of 7-(2-chlorophenylamino)-5-benzyl-1-chloro-6,7-dihydro pyrrolo[2,1-d][1,5]pyrimidodiazepine 4.12a.

Fig. S-44: ${ }^{1} \mathrm{H}$ Spectra of 7-(2-chlorophenylamino)-5-benzyl-1-chloro-6,7-dihydro pyrrolo[2,1-d][1,5]pyrimidodiazepine 4.12a.

Fig. S-45: ${ }^{13} \mathrm{C}$ Spectra of 7-(2-chlorophenylamino)-5-benzyl-1-chloro-6,7-dihydro pyrrolo[2,1-d][1,5]pyrimidodiazepine 4.12a. 
Fig. S-46: LC-MS-ELSD of 1-chloro-5,6-dihydro-6-[(benzylamino)methyl]-5-(3chlorophenyl)pyrrolo[1,2-f]pteridine 5.13a.

Fig. S-47: ${ }^{1} \mathrm{H}$ Spectra of 1-chloro-5,6-dihydro-6-[(benzylamino)methyl]-5-(3-chloro phenyl)pyrrolo[1,2-f]pteridine 5.13a.

Fig. S-48: ${ }^{13} \mathrm{C}$ Spectra of 1-chloro-5,6-dihydro-6-[(benzylamino)methyl]-5-(3-chloro phenyl)pyrrolo[1,2-f]pteridine 5.13a.

Fig. S-49: LC-MS-ELSD of 1-chloro-5,6-dihydro-6-[(benzylamino)methyl]-5-(4chlorophenyl)pyrrolo[1,2-f]pteridine 5.14a.

Fig. S-50: ${ }^{1} \mathrm{H}$ Spectra of 1-chloro-5,6-dihydro-6-[(benzylamino)methyl]-5-(4chlorophenyl)pyrrolo[1,2-f]pteridine 5.14a.

Fig. S-51: ${ }^{13} \mathrm{C}$ Spectra of 1-chloro-5,6-dihydro-6-[(benzylamino)methyl]-5-(4chlorophenyl)pyrrolo[1,2-f]pteridine 5.14a.

Fig. S-52: LC-MS-ELSD of 1-chloro-5,6-dihydro-6-[(benzylamino)methyl]-5-(4-floro phenyl)pyrrolo[1,2-f]pteridine 5.15a.

Fig. S-53: ${ }^{1} \mathrm{H}$ Spectra of 1-chloro-5,6-dihydro-6-[(benzylamino)methyl]-5-(4-floro phenyl)pyrrolo[1,2-f]pteridine 5.15a.

Fig. S-54: ${ }^{13} \mathrm{C}$ Spectra of 1-chloro-5,6-dihydro-6-[(benzylamino)methyl]-5-(4-floro phenyl)pyrrolo[1,2-f]pteridine 5.15a.

Fig. S-55: LC-MS-ELSD of 7-(2-nitrophenylamino)-5-benzyl-1-chloro-6,7-dihydro pyrrolo[2,1-d][1,5]pyrimidodiazepine 4.16a.

Fig. S-56: ${ }^{1} \mathrm{H}$ Spectra of 7-(2-nitrophenylamino)-5-benzyl-1-chloro-6,7-dihydro pyrrolo[2,1-d][1,5]pyrimidodiazepine 4.16a.

Fig. S-57: ${ }^{13} \mathrm{C}$ Spectra of 7-(2-nitrophenylamino)-5-benzyl-1-chloro-6,7-dihydro pyrrolo[2,1-d][1,5]pyrimidodiazepine 4.16a.

Fig. S-58: LC-MS-ELSD of 1-chloro-5,6-dihydro-6-[(benzylamino)methyl]-5-(4-nitro phenyl)pyrrolo[1,2-f]pteridine 5.17a.

Fig. S-59: ${ }^{1} \mathrm{H}$ Spectra of 1-chloro-5,6-dihydro-6-[(benzylamino)methyl]-5-(4-nitro phenyl)pyrrolo[1,2-f]pteridine 5.17a.

Fig. S-60: ${ }^{13} \mathrm{C}$ Spectra of 1-chloro-5,6-dihydro-6-[(benzylamino)methyl]-5-(4-nitro phenyl)pyrrolo[1,2-f]pteridine 5.17a. 
Fig. S-61: LC-MS-ELSD of 1-chloro-5,6-dihydro-5-[benzylhydroxyl]-6[(benzylamino)methyl]pyrrolo[1,2-f]pteridine 5.18a.

Fig. S-62: ${ }^{1} \mathrm{H}$ Spectra of 1-chloro-5,6-dihydro-5-[benzylhydroxyl]-6-[(benzylamino) methyl]pyrrolo[1,2-f]pteridine 5.18a.

Fig. S-63: ${ }^{13} \mathrm{C}$ Spectra of 1-chloro-5,6-dihydro-5-[benzylhydroxyl]-6-[(benzylamino) methyl]pyrrolo[1,2-f]pteridine 5.18a.

Fig. S-64: LC-MS-ELSD of 1-chloro-5,6-dihydro-6-[(methylamino)methyl]-5-(4methylphenyl)pyrrolo[1,2-f]pteridine 5.10b.

Fig. S-65: ${ }^{1} \mathrm{H}$ Spectra of 1-chloro-5,6-dihydro-6-[(methylamino)methyl]-5-(4-methyl phenyl)pyrrolo[1,2-f]pteridine 5.10b.

Fig. S-66: ${ }^{13} \mathrm{C}$ Spectra of 1-chloro-5,6-dihydro-6-[(methylamino)methyl]-5-(4-methyl phenyl)pyrrolo[1,2-f]pteridine 5.10b.

Fig. S-67: LC-MS-ELSD of 1-chloro-5,6-dihydro-6-[(methylamino)methyl]-5-(4chlorophenyl)pyrrolo[1,2-f]pteridine $5.14 \mathrm{~b}$.

Fig. S-68: ${ }^{1} \mathrm{H}$ Spectra of 1-chloro-5,6-dihydro-6-[(methylamino)methyl]-5-(4-chloro phenyl)pyrrolo[1,2-f]pteridine 5.14b.

Fig. S-69: ${ }^{13} \mathrm{C}$ Spectra of 1-chloro-5,6-dihydro-6-[(methylamino)methyl]-5-(4-chloro phenyl)pyrrolo[1,2-f]pteridine 5.14b.

Fig. S-70: LC-MS-ELSD of 5-benzyl-1-chloro-6,7-dihydro-7-hydroxylpyrrolo[2,1d][1,5]pyrimidodiazepine 6 a.

Fig. S-71: ${ }^{1} \mathrm{H}$ Spectra of 5-benzyl-1-chloro-6,7-dihydro-7-hydroxylpyrrolo[2,1d][1,5]pyrimidodiazepine 6 a.

Fig. S-72: ${ }^{13} \mathrm{C}$ Spectra of 5-benzyl-1-chloro-6,7-dihydro-7-hydroxylpyrrolo[2,1d][1,5]pyrimidodiazepine 6 . 
<smiles>OCCN(Cc1ccccc1)c1ncnc(Cl)c1-n1cccc1</smiles>

$\mathrm{C}_{17} \mathrm{H}_{17} \mathrm{ClN}_{4} \mathrm{O}$

Exact Mass: 328.11

2a Mol. Wt.: 328.8
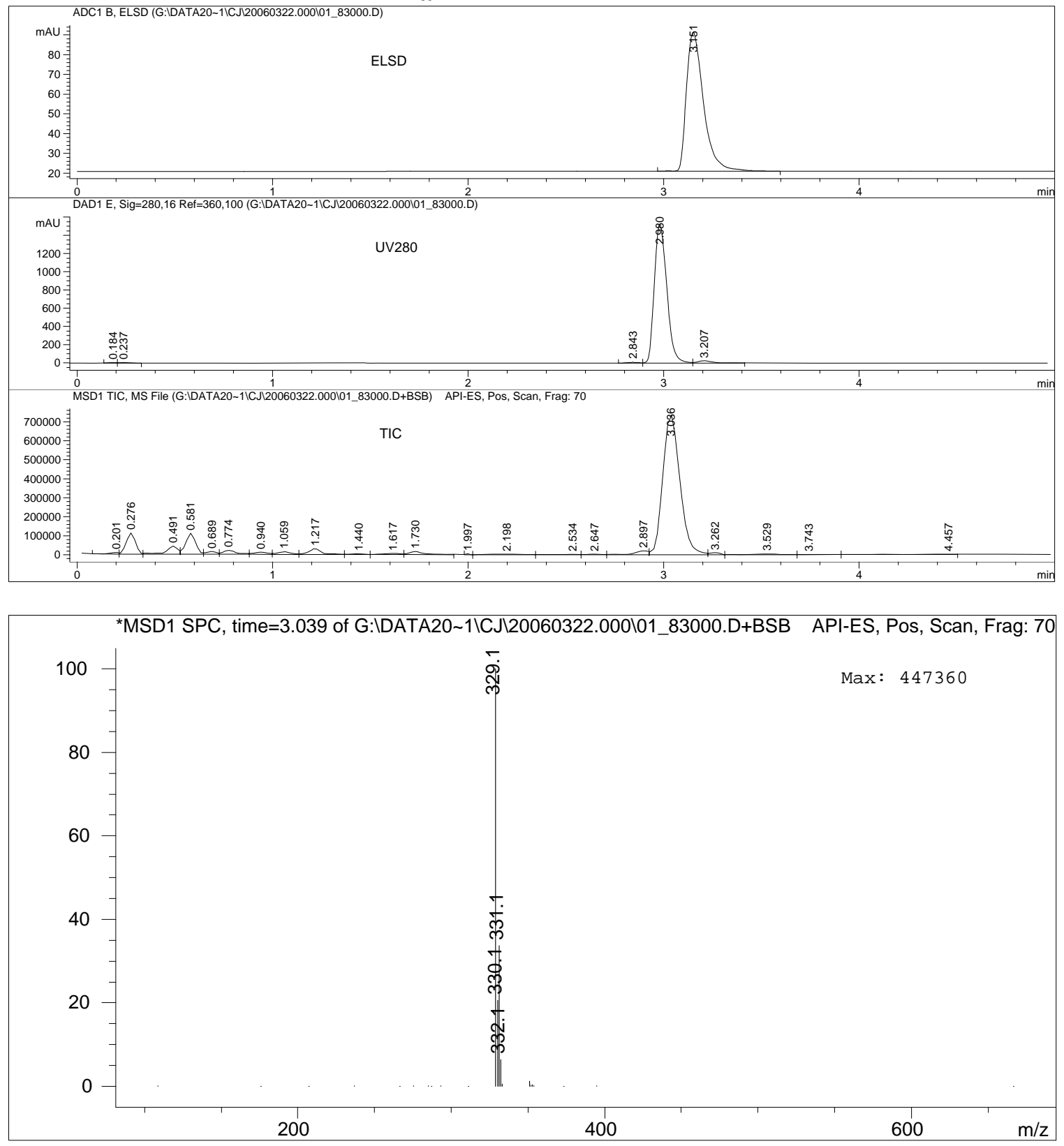

Fig. S-1: LC-MS-ELSD of 6-[N-benzyl(2-ethanol)amino]-4-chloro-5-pyrrol-1-ylpyrimidine $2 a$. 


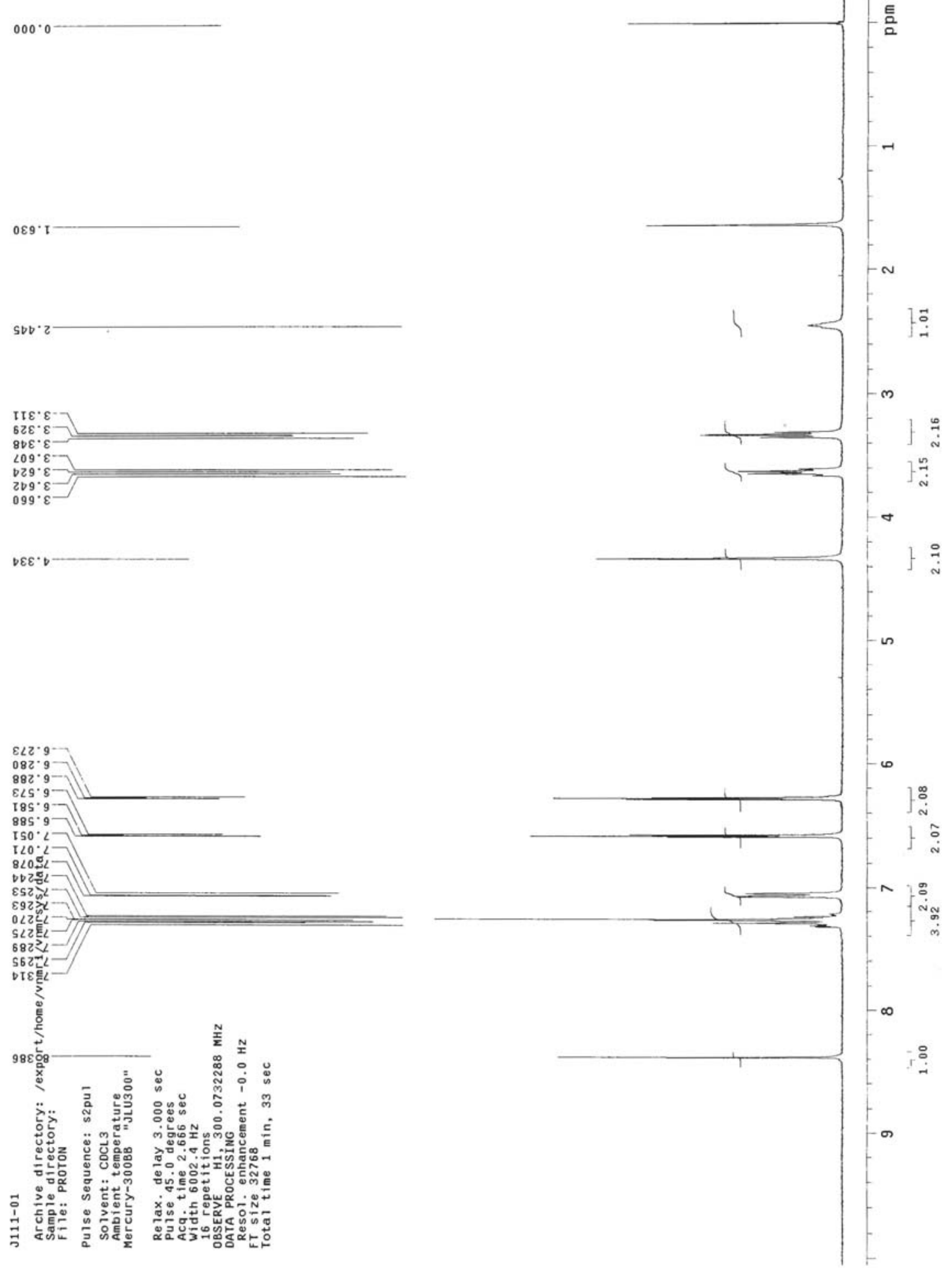

Fig. S-2: ${ }^{1} \mathrm{H} \quad$ Spectra of $\quad$ 6-[ $N$-benzyl(2-ethanol)amino]-4-chloro-5-pyrrol-1-ylpyrimidine $2 \mathbf{a}$. 


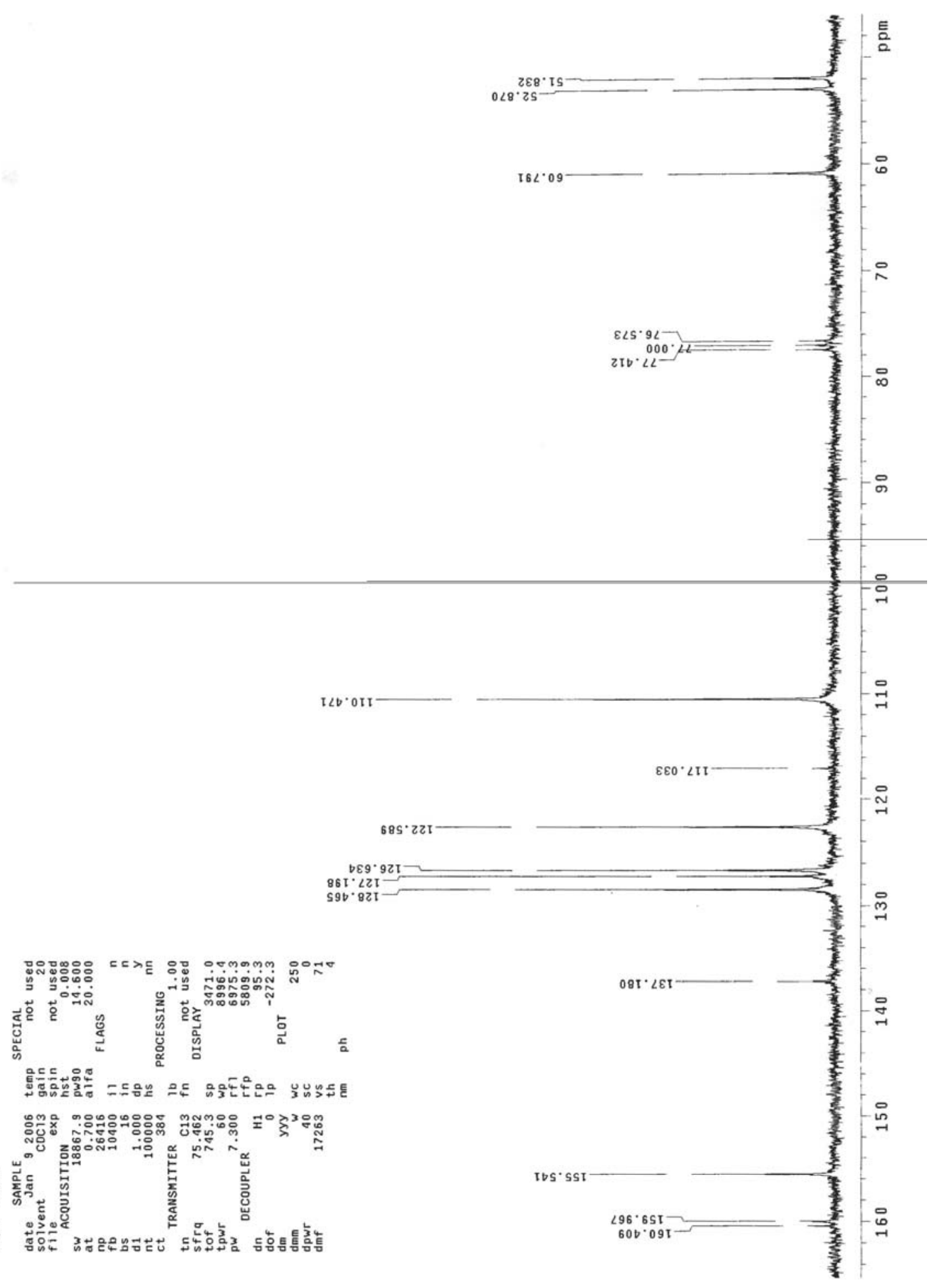

Fig. S-3: ${ }^{13} \mathrm{C}$ Spectra of 6-[N-benzyl(2-ethanol)amino]-4-chloro-5-pyrrol-1-ylpyrimidine 2 a. 

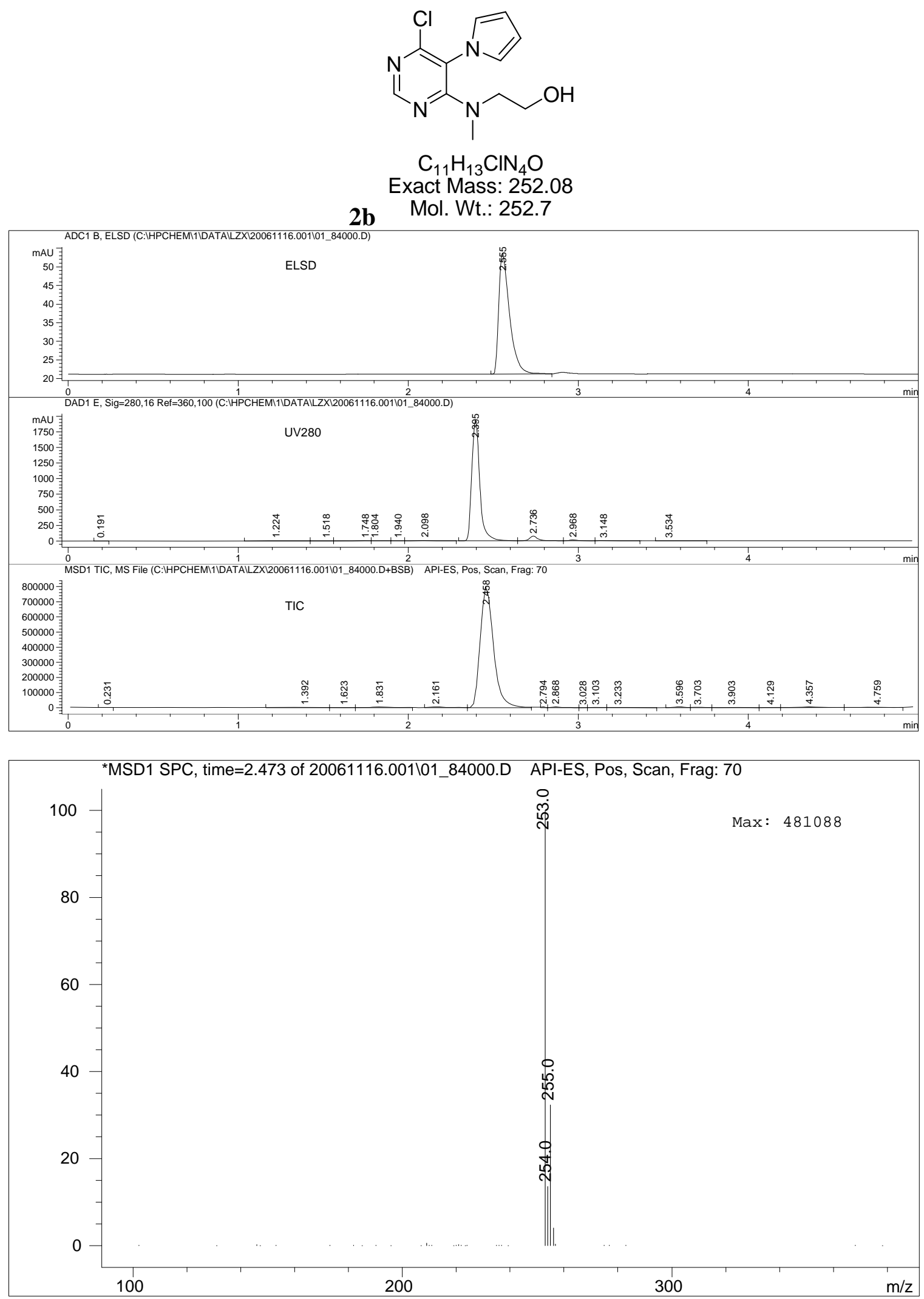

Fig. S-4: LC-MS-ELSD of 6-[ $N$-methyl(2-ethanol)amino]-4-chloro-5-pyrrol-1-ylpyrimidine $2 \mathbf{b}$. 


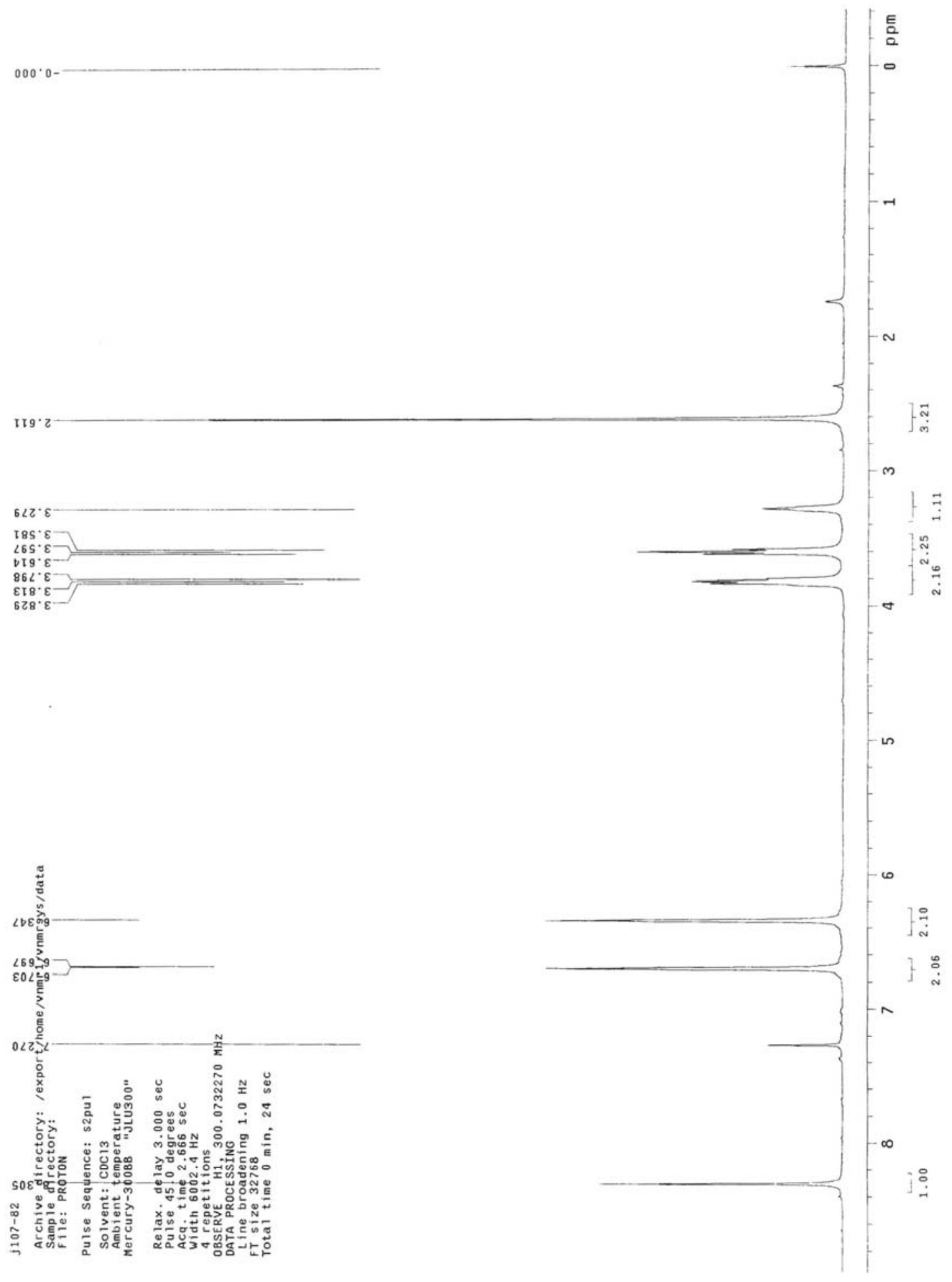

Fig. S-5: ${ }^{1} \mathrm{H} \quad$ Spectra of $\quad 6-[N$-methyl(2-ethanol)amino]-4-chloro-5-pyrrol-1-ylpyrimidine $2 \mathbf{b}$. 


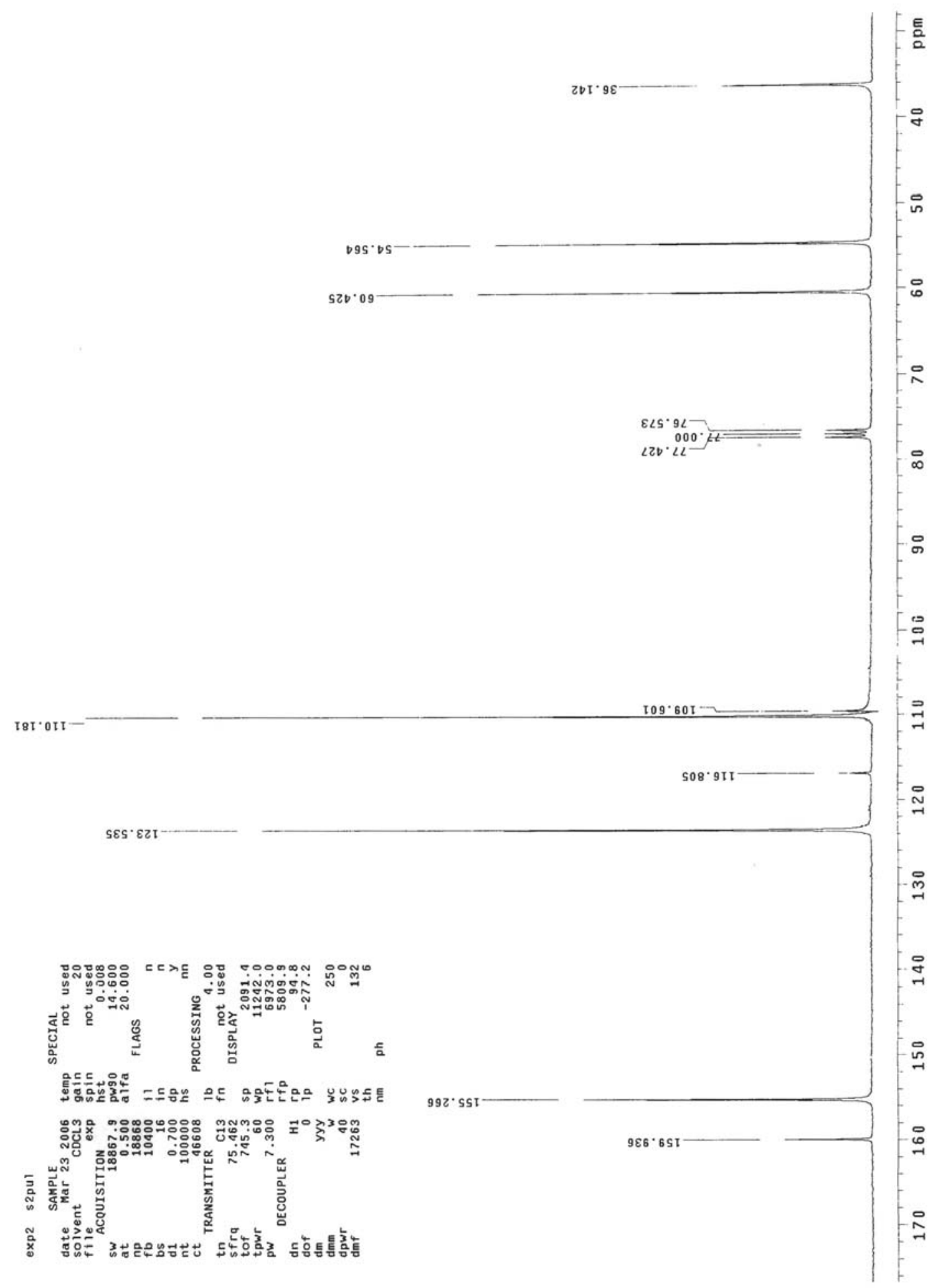

Fig. S-6: ${ }^{13} \mathrm{C}$ Spectra of 6-[ $N$-methyl(2-ethanol)amino]-4-chloro-5-pyrrol-1-ylpyrimidine $2 \mathbf{b}$. 
<smiles>CN1c2ncnc(Cl)c2-n2cccc2C1CNCc1ccccc1</smiles>

$\mathrm{C}_{18} \mathrm{H}_{18} \mathrm{ClN}_{5}$

Exact Mass: 339.13

5.1a

Mol. Wt.: 339.82
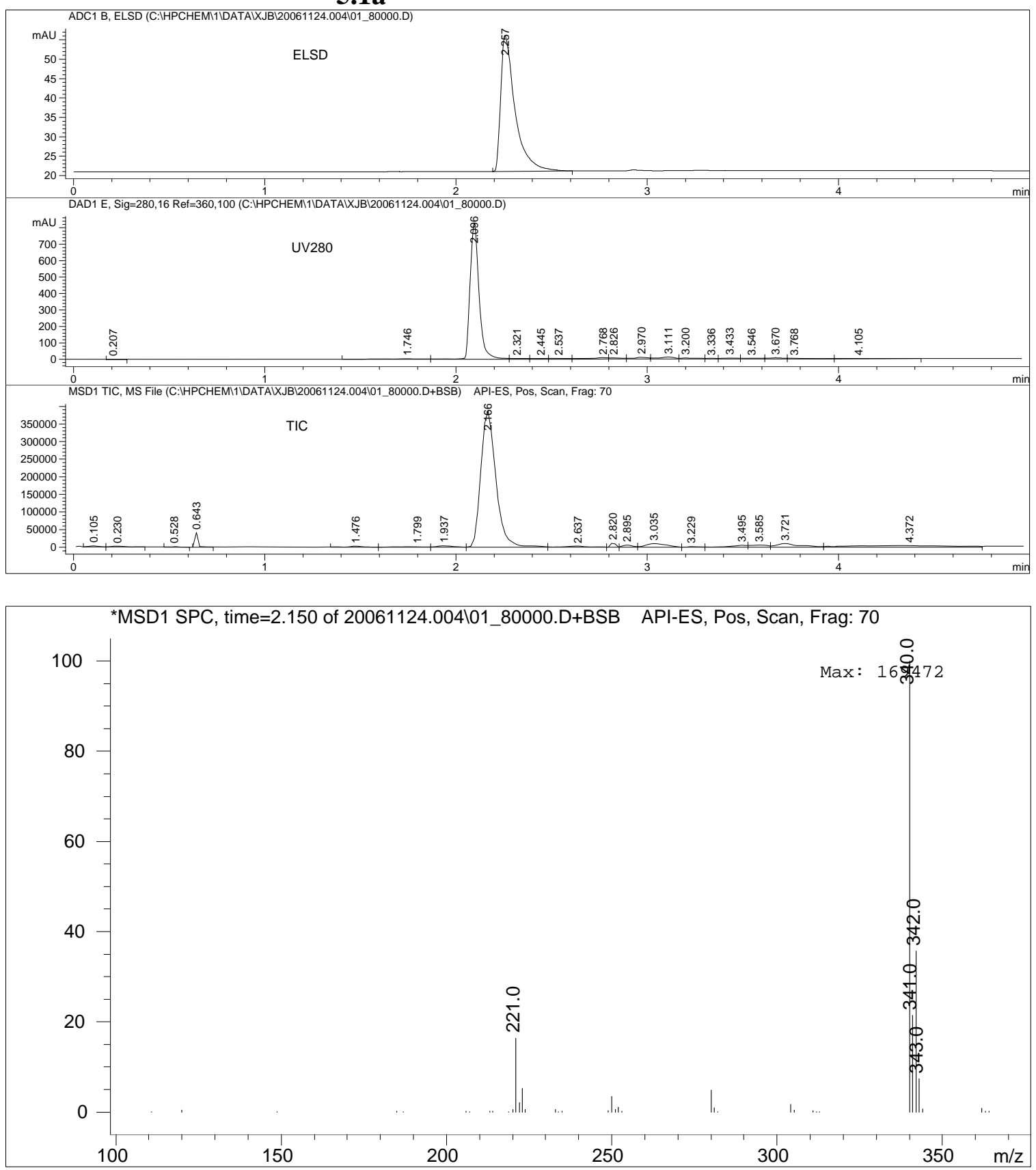

Fig. S-7: LC-MS-ELSD of 1-chloro-5,6-dihydro-5-methyl-6-[(benzylamino)methyl] pyrrolo[1,2-f]pteridine 5.1a. 


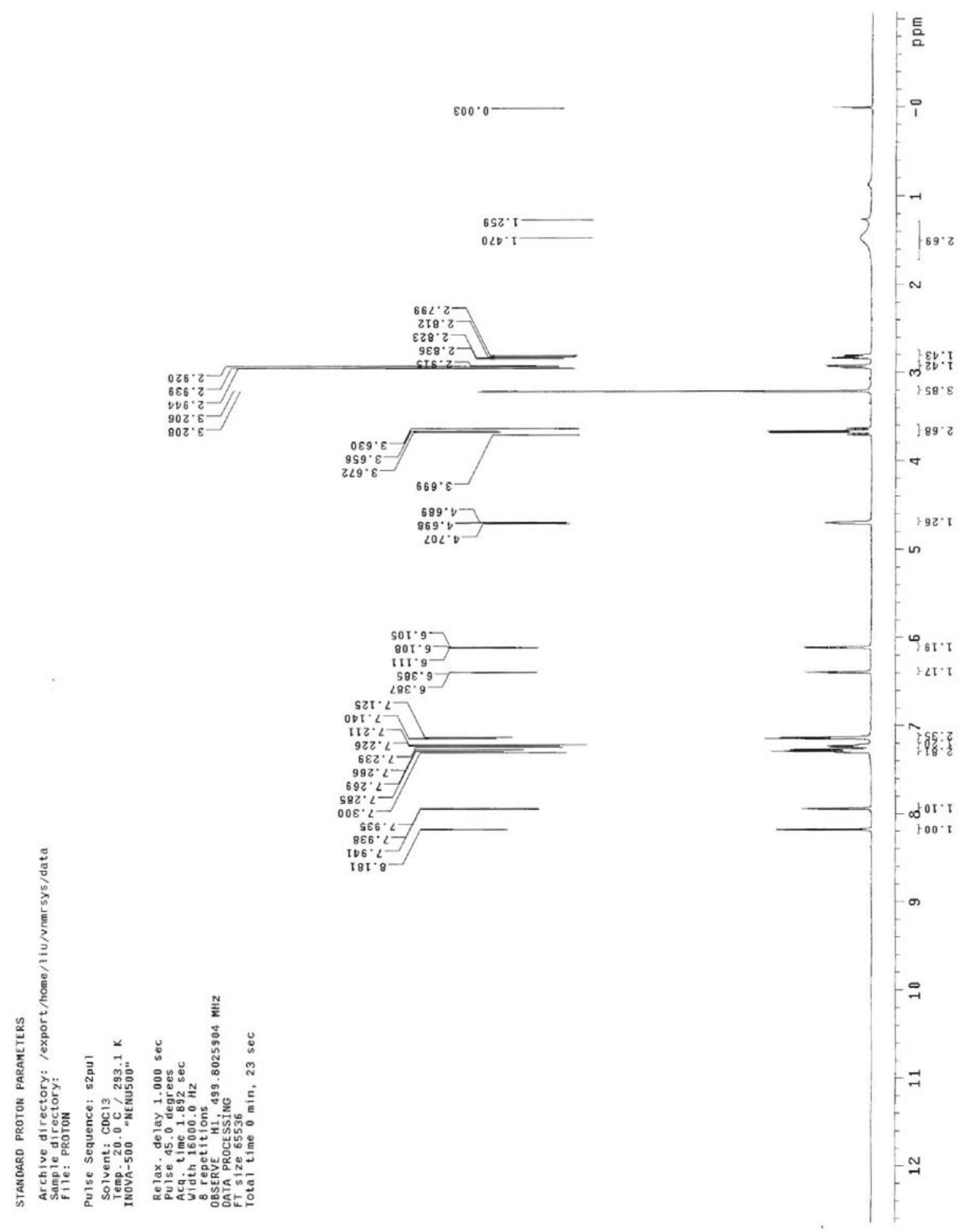

Fig. S-8: ${ }^{1} \mathrm{H}$ Spectra of 1-chloro-5,6-dihydro-5-methyl-6-[(benzylamino)methyl] pyrrolo[1,2-f]pteridine 5.1a. 


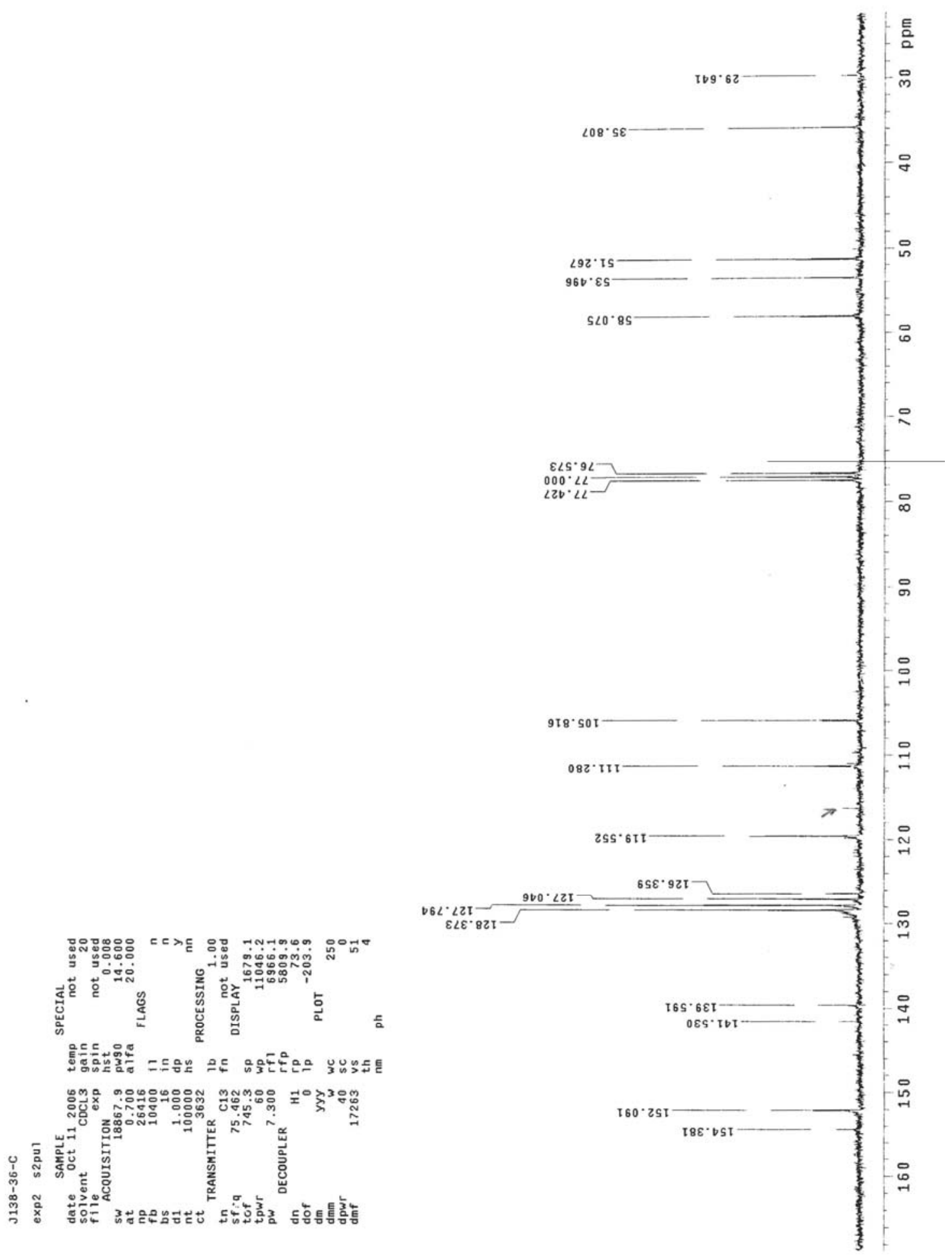

Fig. S-9: ${ }^{13} \mathrm{C}$ Spectra of 1-chloro-5,6-dihydro-5-methyl-6-[(benzylamino)methyl] pyrrolo[1,2-f]pteridine 5.1a. 

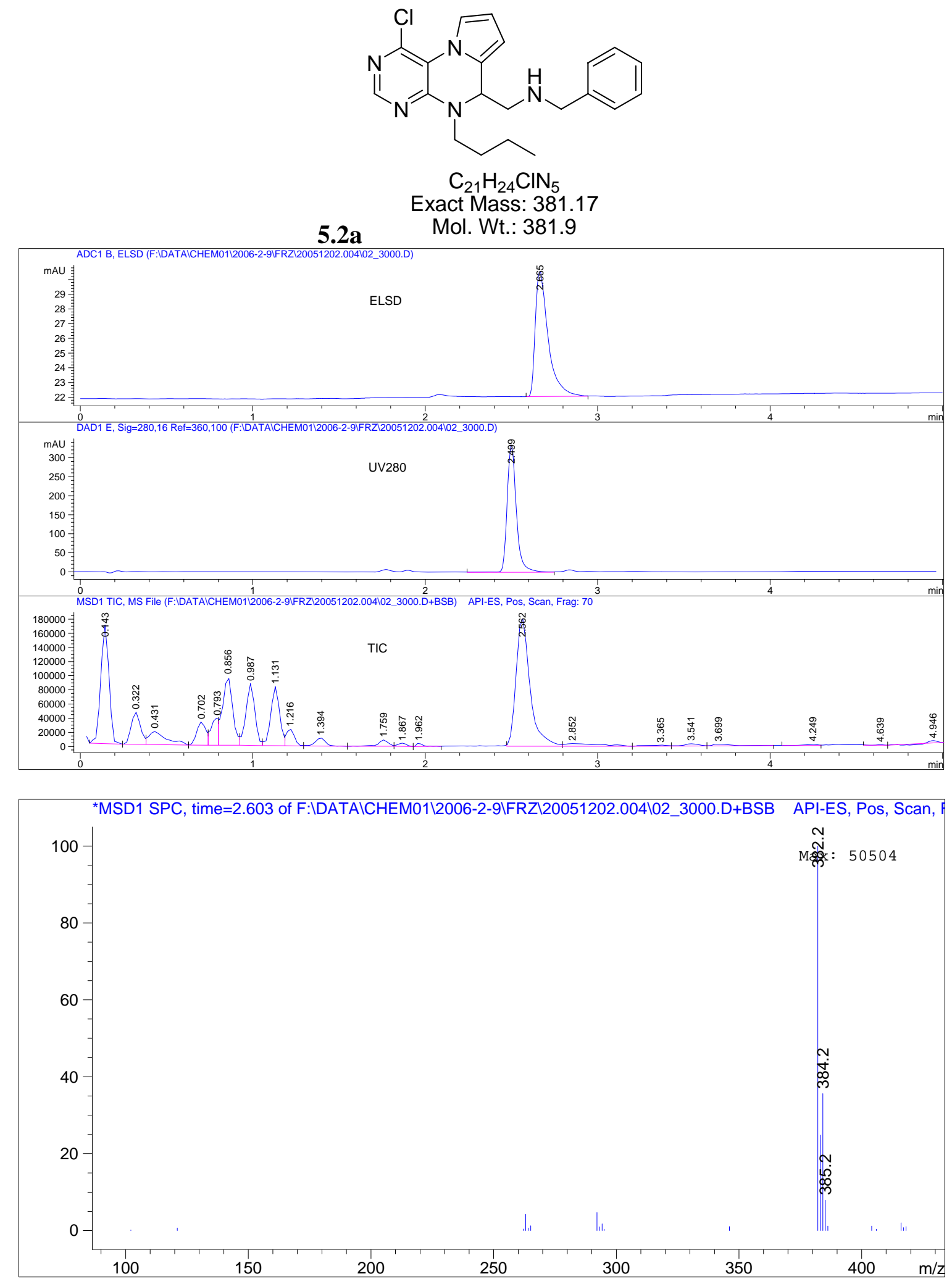

Fig. S-10: LC-MS-ELSD of 5-(n-butyl)-1-chloro-5,6-dihydro-6-[(benzylamino)methyl] pyrrolo[1,2-f]pteridine 5.2a. 
600.00
000
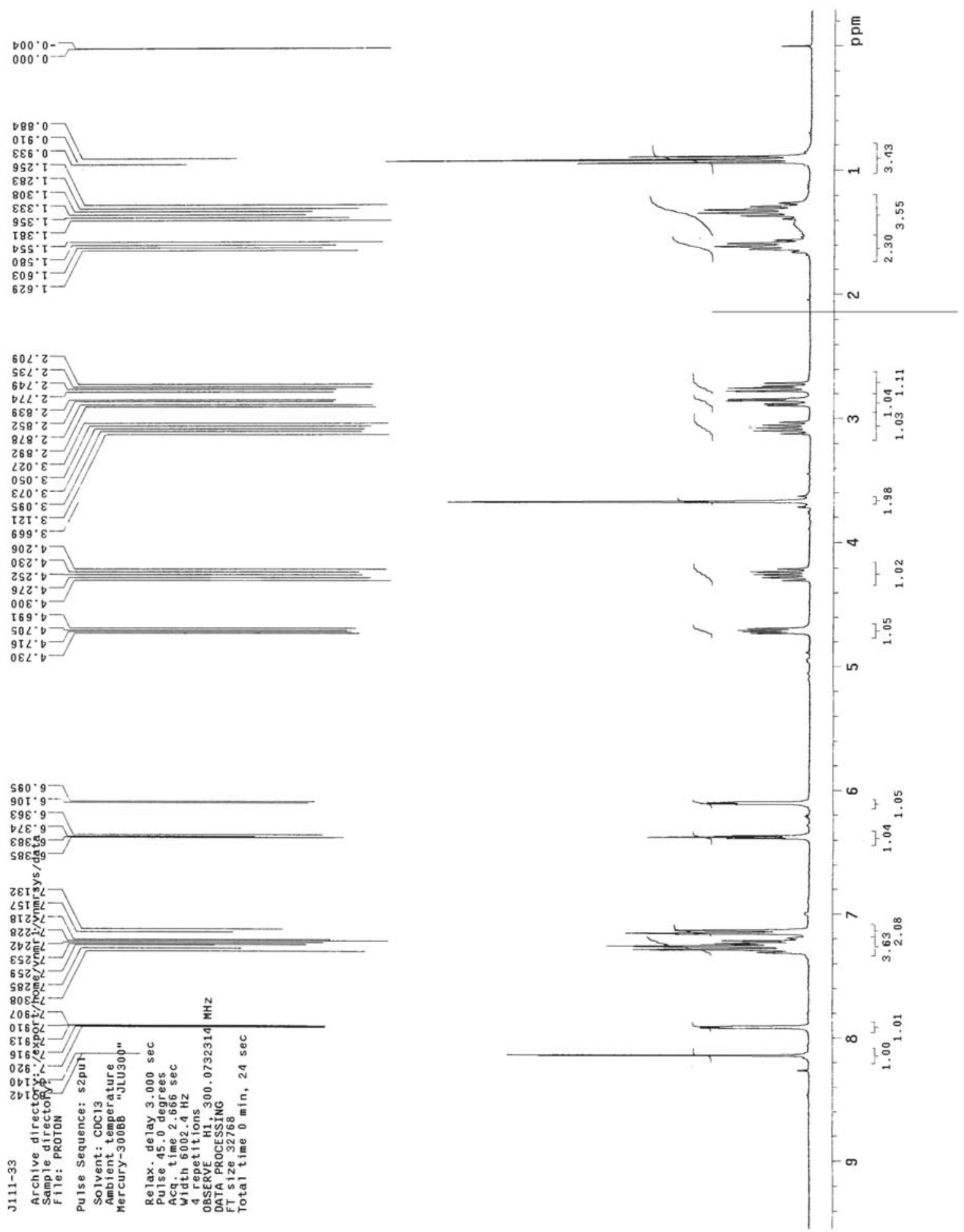

Fig. S-11: ${ }^{1} \mathrm{H}$ Spectra of 5-(n-butyl)-1-chloro-5,6-dihydro-6-[(benzylamino)methyl] pyrrolo[1,2-f]pteridine 5.2a. 


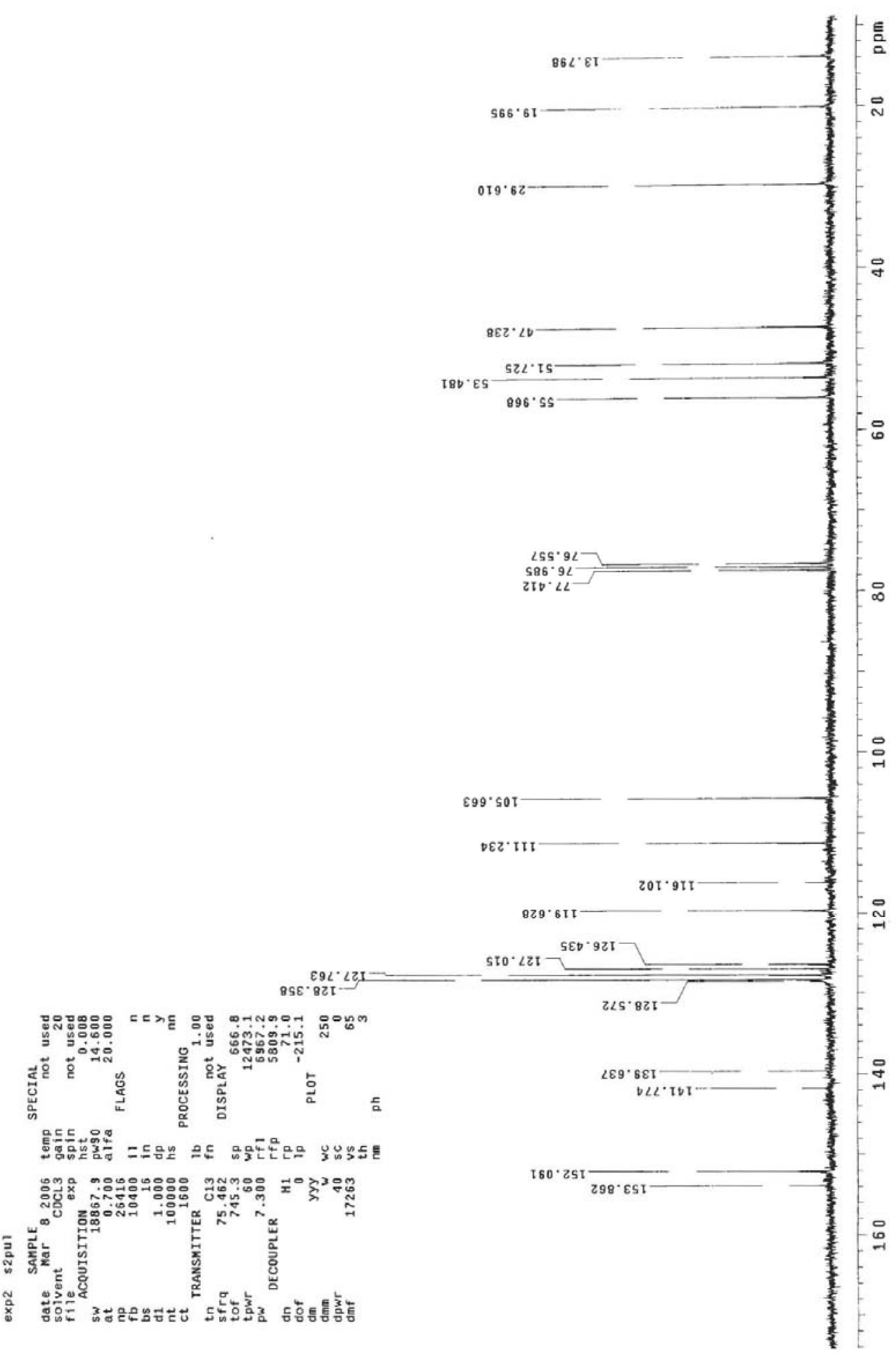

Fig. S-12: ${ }^{13} \mathrm{C}$ Spectra of 5-(n-butyl)-1-chloro-5,6-dihydro-6-[(benzylamino)methyl] pyrrolo[1,2-f]pteridine 5.2a. 


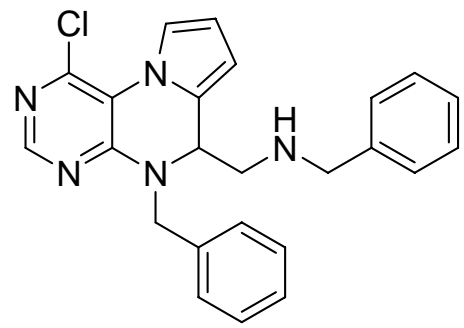

$\mathrm{C}_{24} \mathrm{H}_{22} \mathrm{ClN}_{5}$

Exact Mass: 415.16

5.3a Mol. Wt.: 415.92
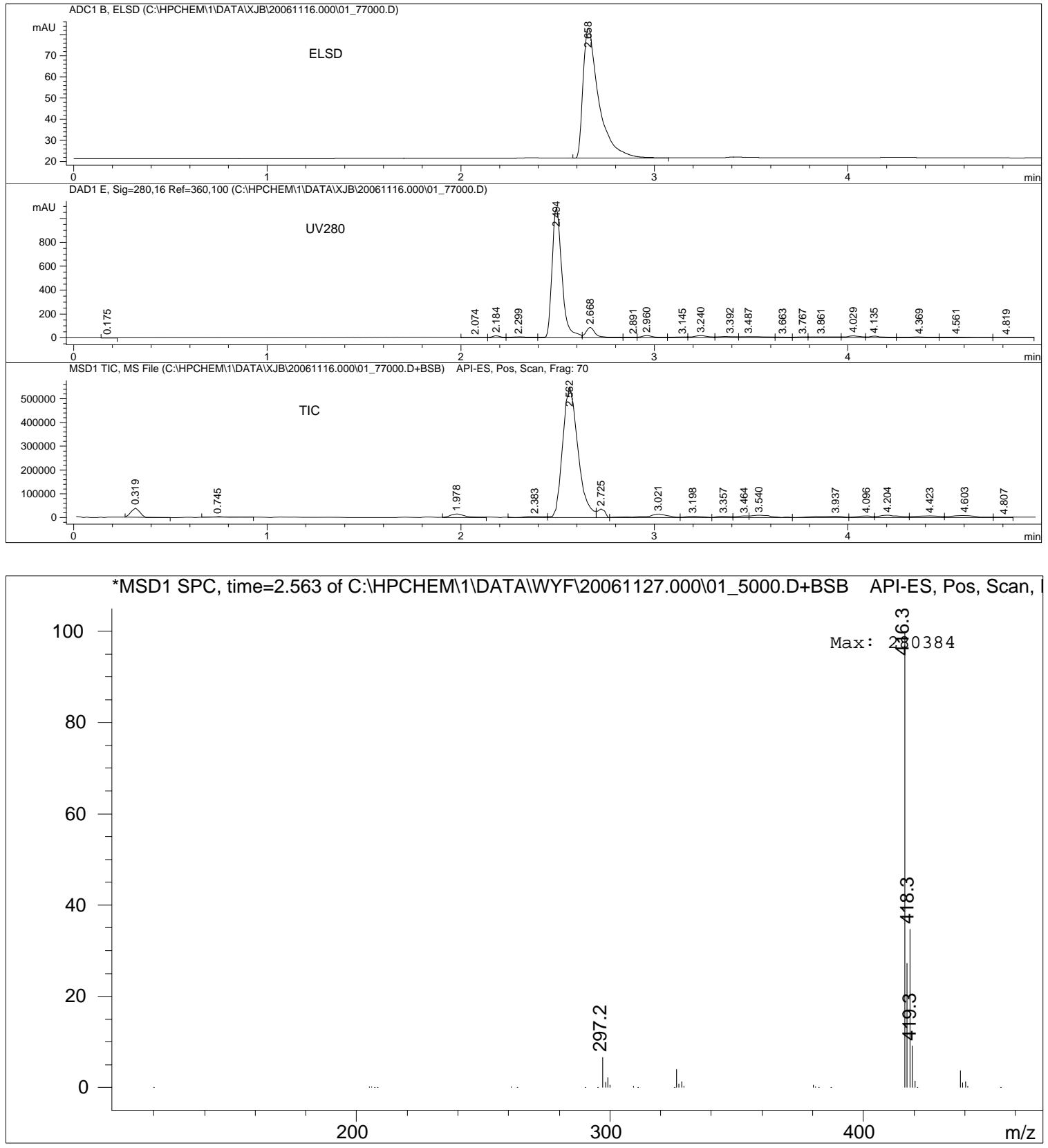

Fig. S-13: LC-MS-ELSD of 5-benzyl-1-chloro-5,6-dihydro-6-[(benzylamino)methyl] pyrrolo[1,2-f]pteridine 5.3a. 


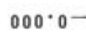

$\forall S Z^{\circ} \mathrm{I}$

$9 \varepsilon \varepsilon \cdot \tau-$

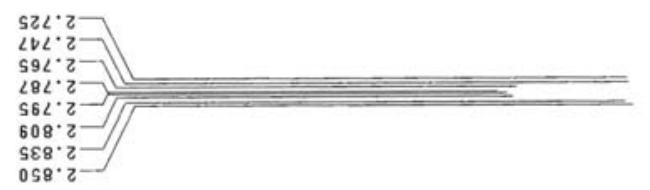

$9+9 \cdot \varepsilon-$
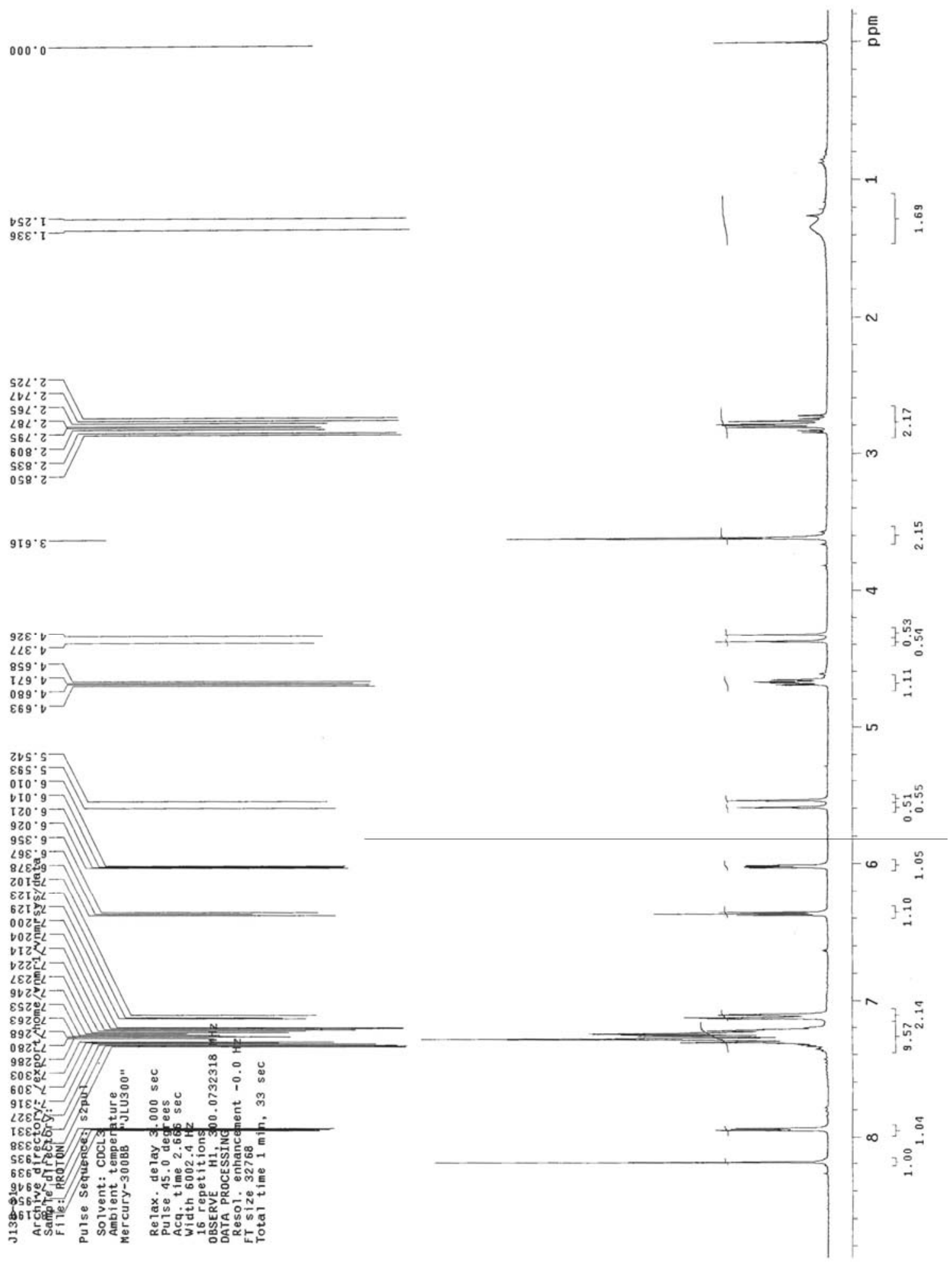

Fig. S-14: ${ }^{1} \mathrm{H}$ Spectra of 5-benzyl-1-chloro-5,6-dihydro-6-[(benzylamino)methyl] pyrrolo[1,2-f]pteridine 5.3a. 


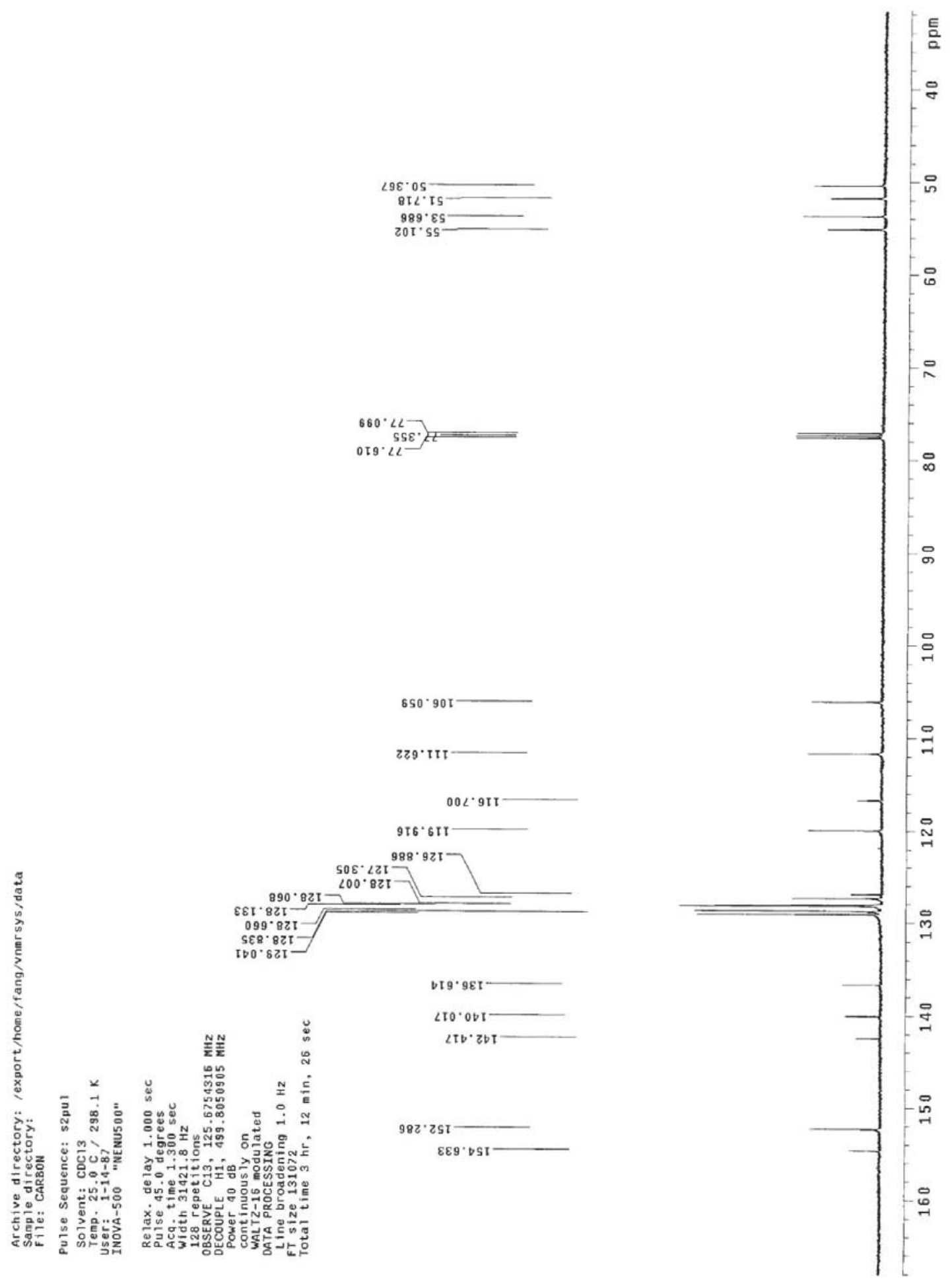

Fig. S-15: ${ }^{13} \mathrm{C}$ Spectra of 5-benzyl-1-chloro-5,6-dihydro-6-[(benzylamino)methyl] pyrrolo[1,2-f]pteridine 5.3a. 

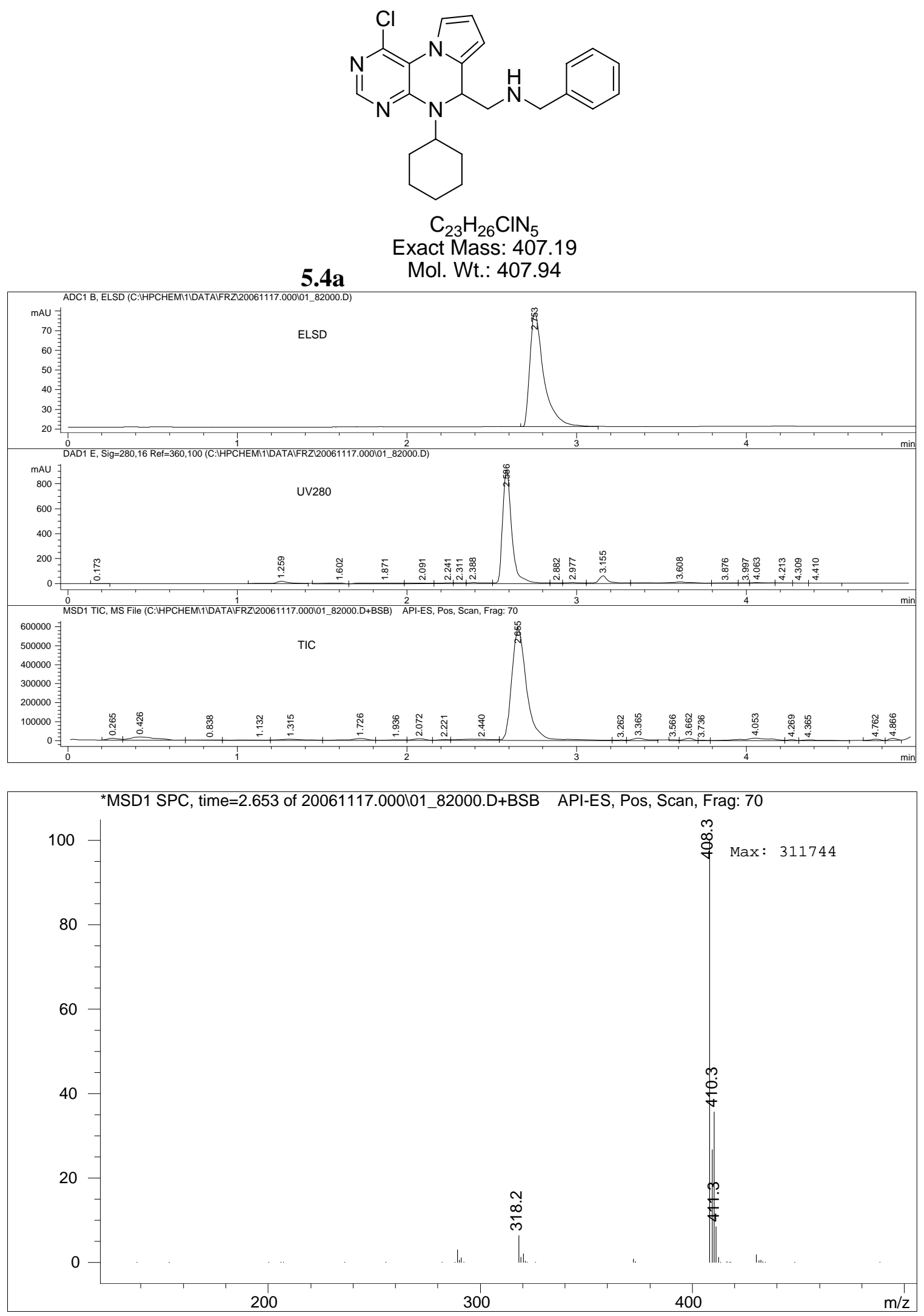

Fig. S-16: LC-MS-ELSD of 1-chloro-5-cyclohexyl-5,6-dihydro-6-[(benzylamino) methyl]pyrrolo[1,2-f]pteridine 5.4a. 


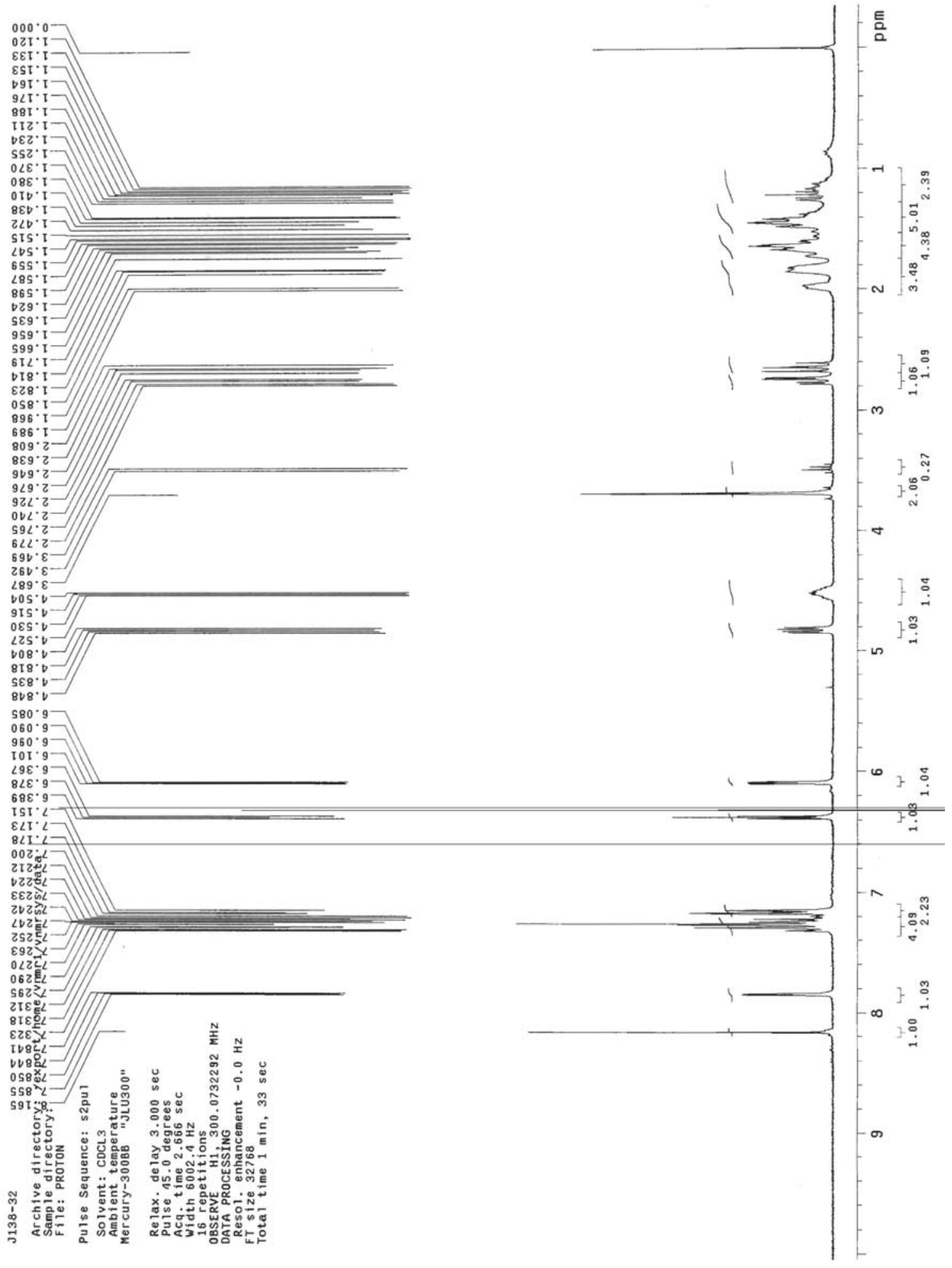

Fig. S-17: ${ }^{1} \mathrm{H}$ Spectra of 1-chloro-5-cyclohexyl-5,6-dihydro-6-[(benzylamino)methyl] pyrrolo[1,2-f]pteridine 5.4a. 


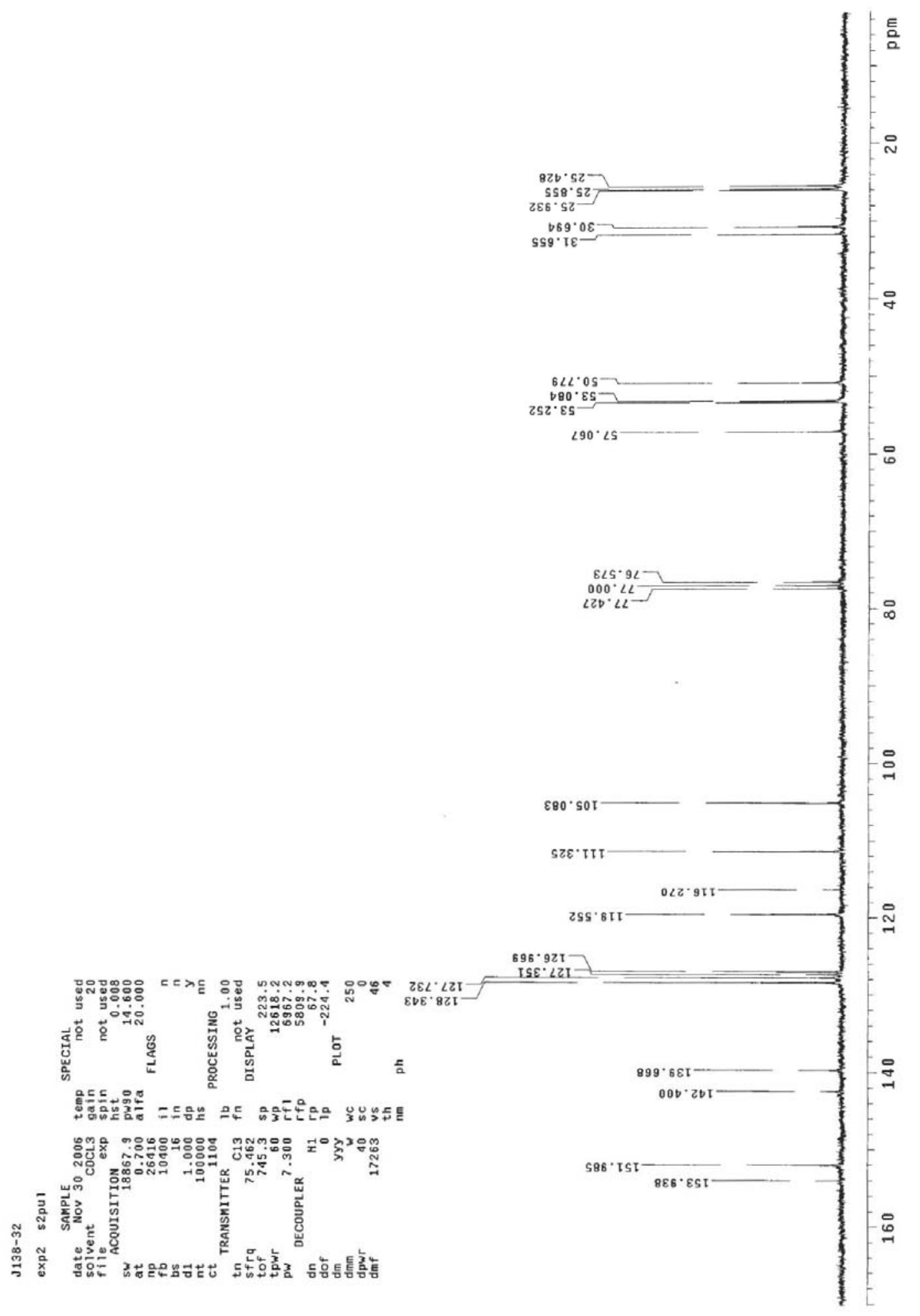

Fig. S-18: ${ }^{13} \mathrm{C}$ Spectra of 1-chloro-5-cyclohexyl-5,6-dihydro-6-[(benzylamino)methyl] pyrrolo[1,2-f]pteridine 5.4a. 

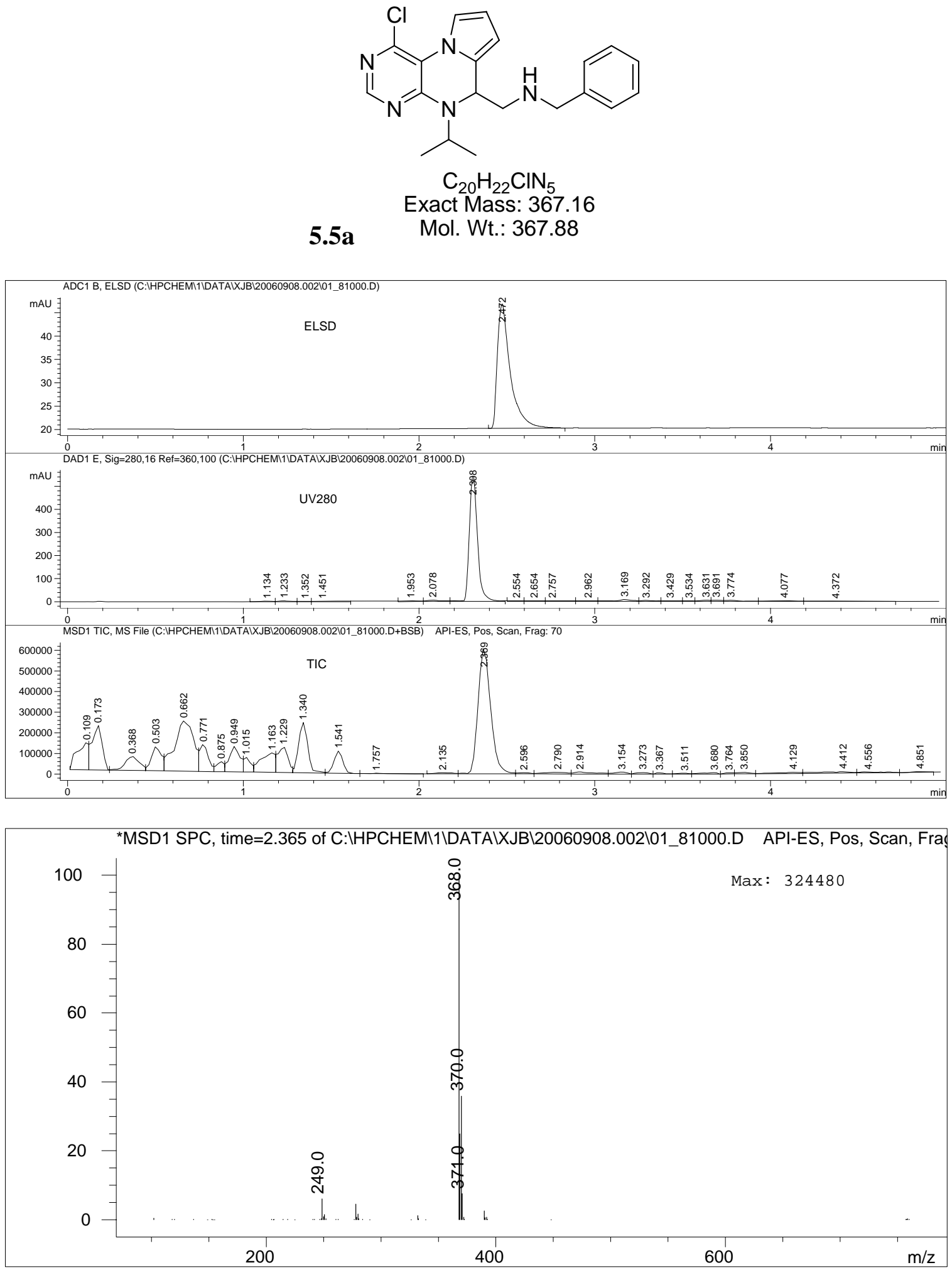

Fig. S-19: LC-MS-ELSD of 1-chloro-5,6-dihydro-6-[(benzylamino)methyl]-5-(isopropyl)pyrrolo[1,2-f]pteridine 5.5a. 


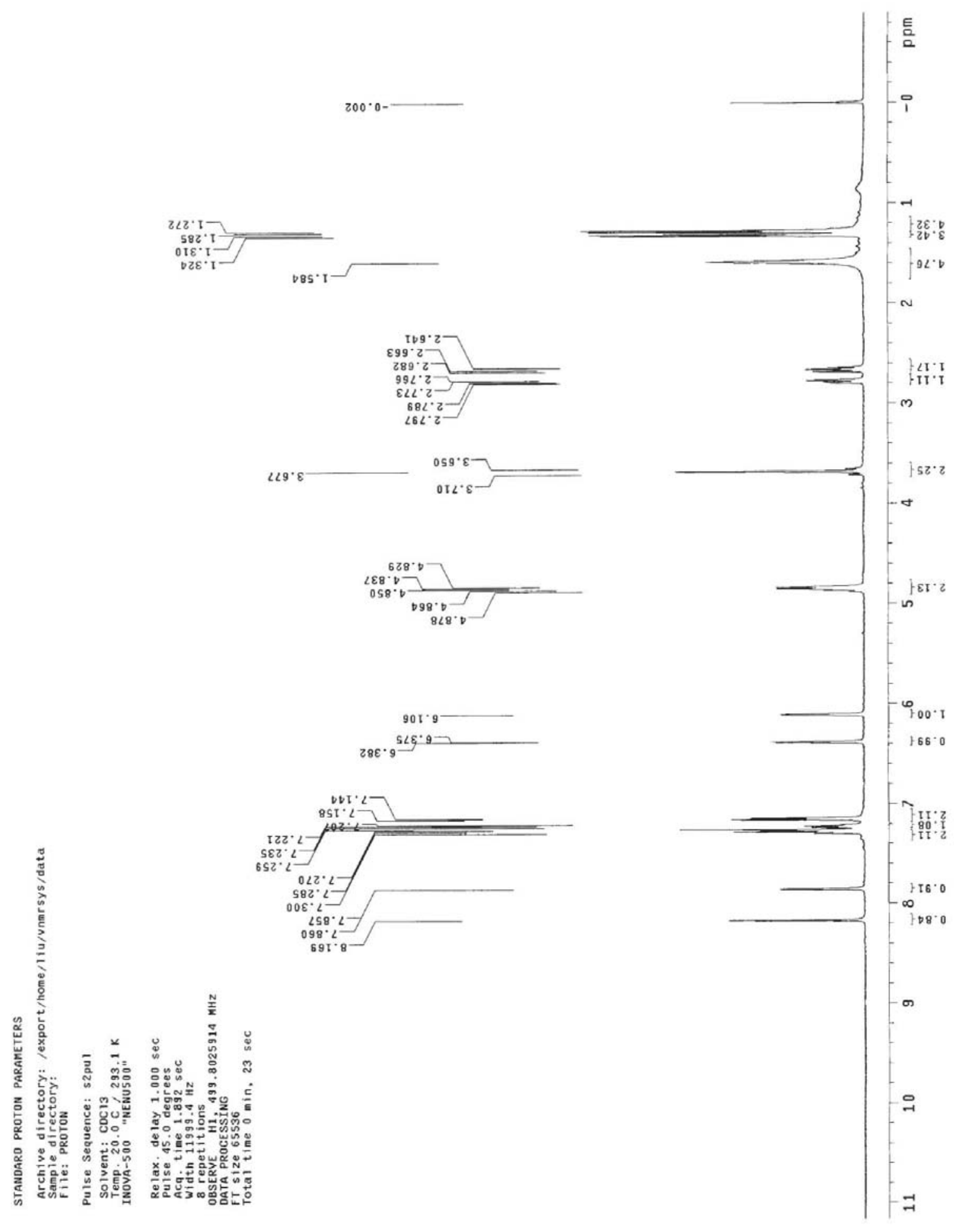

Fig. S-20: ${ }^{1} \mathrm{H}$ Spectra of 1-chloro-5,6-dihydro-6-[(benzylamino)methyl]-5-(isopropyl)pyrrolo[1,2-f]pteridine 5.5a. 


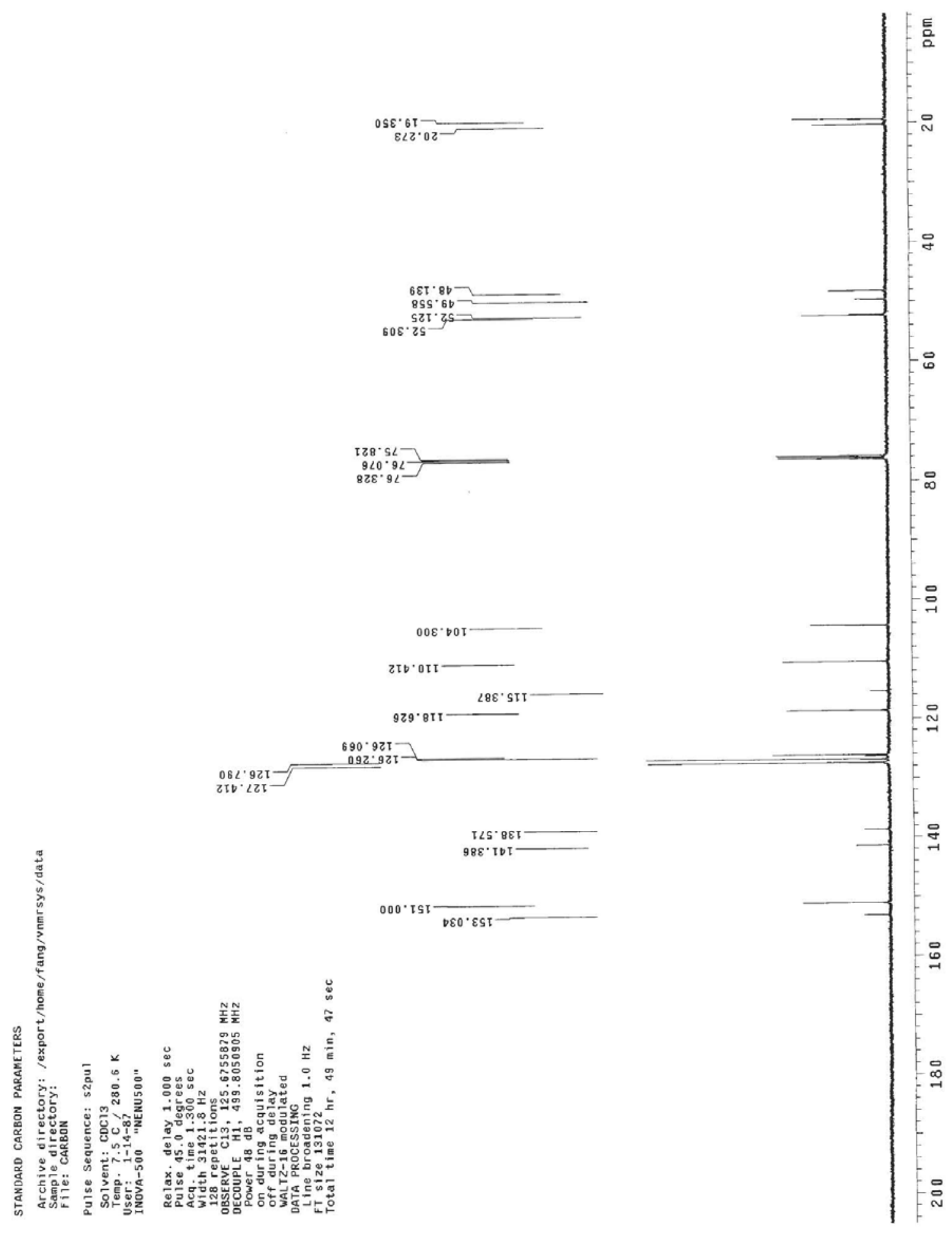

Fig. S-21: ${ }^{13} \mathrm{C}$ Spectra of 1-chloro-5,6-dihydro-6-[(benzylamino)methyl]-5-(isopropyl)pyrrolo[1,2-f]pteridine 5.5a. 
<smiles>COc1ccccc1N1c2ncnc(Cl)c2-n2cccc2C1CNCc1ccccc1</smiles>

$\mathrm{C}_{24} \mathrm{H}_{22} \mathrm{ClN}_{5} \mathrm{O}$

Exact Mass: 431.15

5.6a Mol. Wt.: 431.92
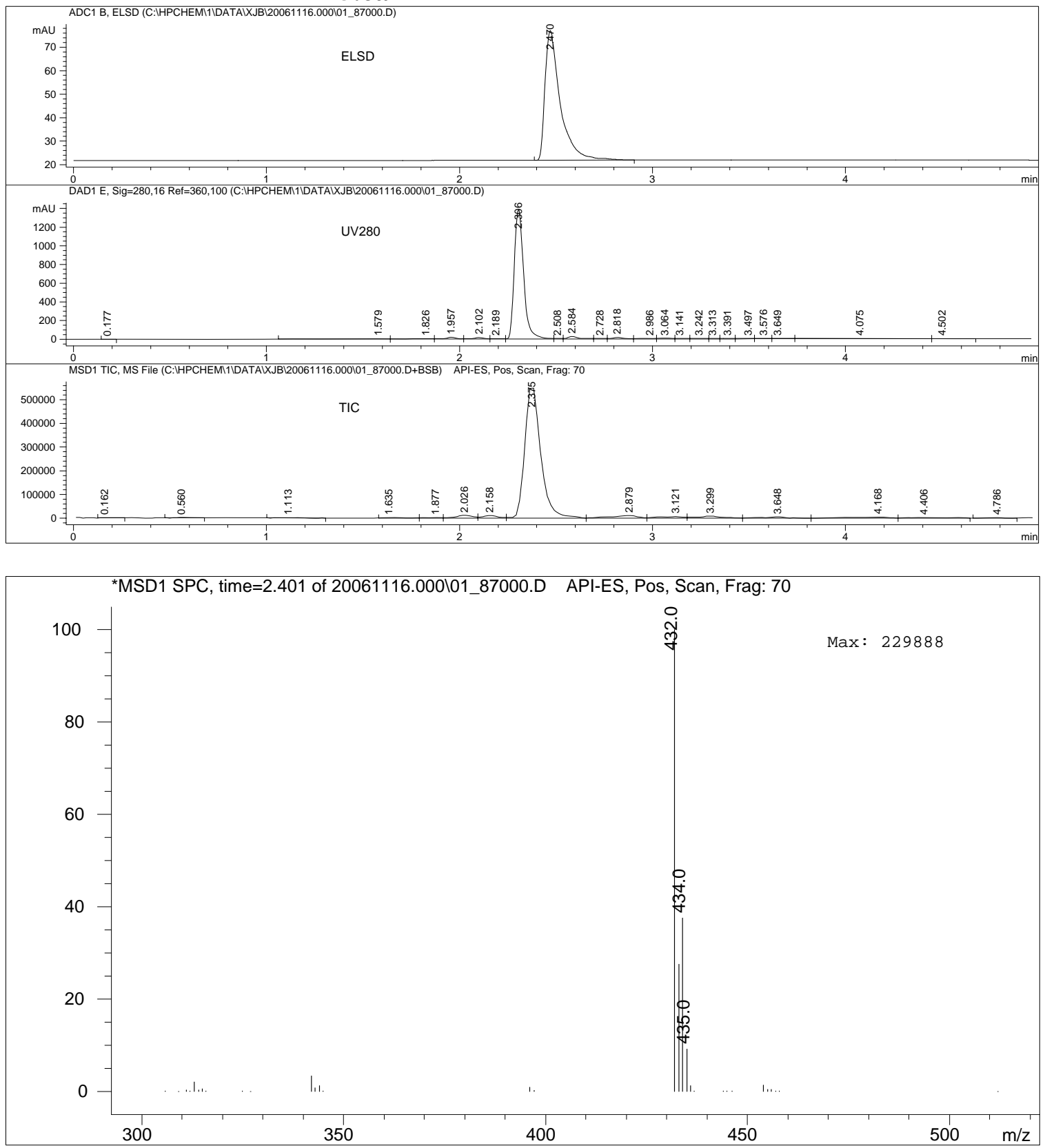

Fig. S-22: LC-MS-ELSD of 1-chloro-5,6-dihydro-6-[(benzylamino)methyl]-5-(2methoxylphenyl)pyrrolo[1,2-f]pteridine 5.6a. 


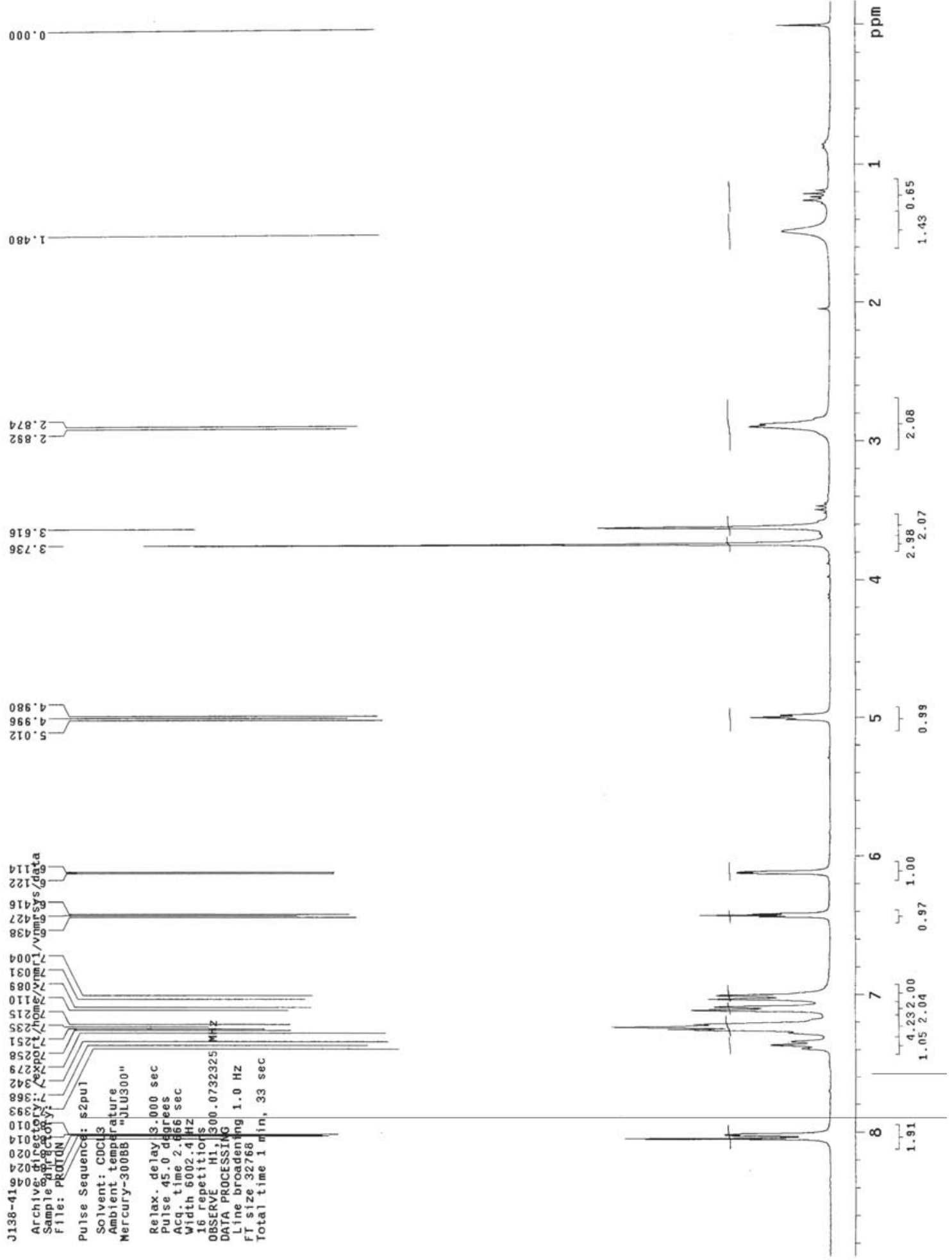

Fig. S-23: ${ }^{1} \mathrm{H}$ Spectra of 1-chloro-5,6-dihydro-6-[(benzylamino)methyl]-5-(2methoxylphenyl)pyrrolo[1,2-f]pteridine 5.6a. 


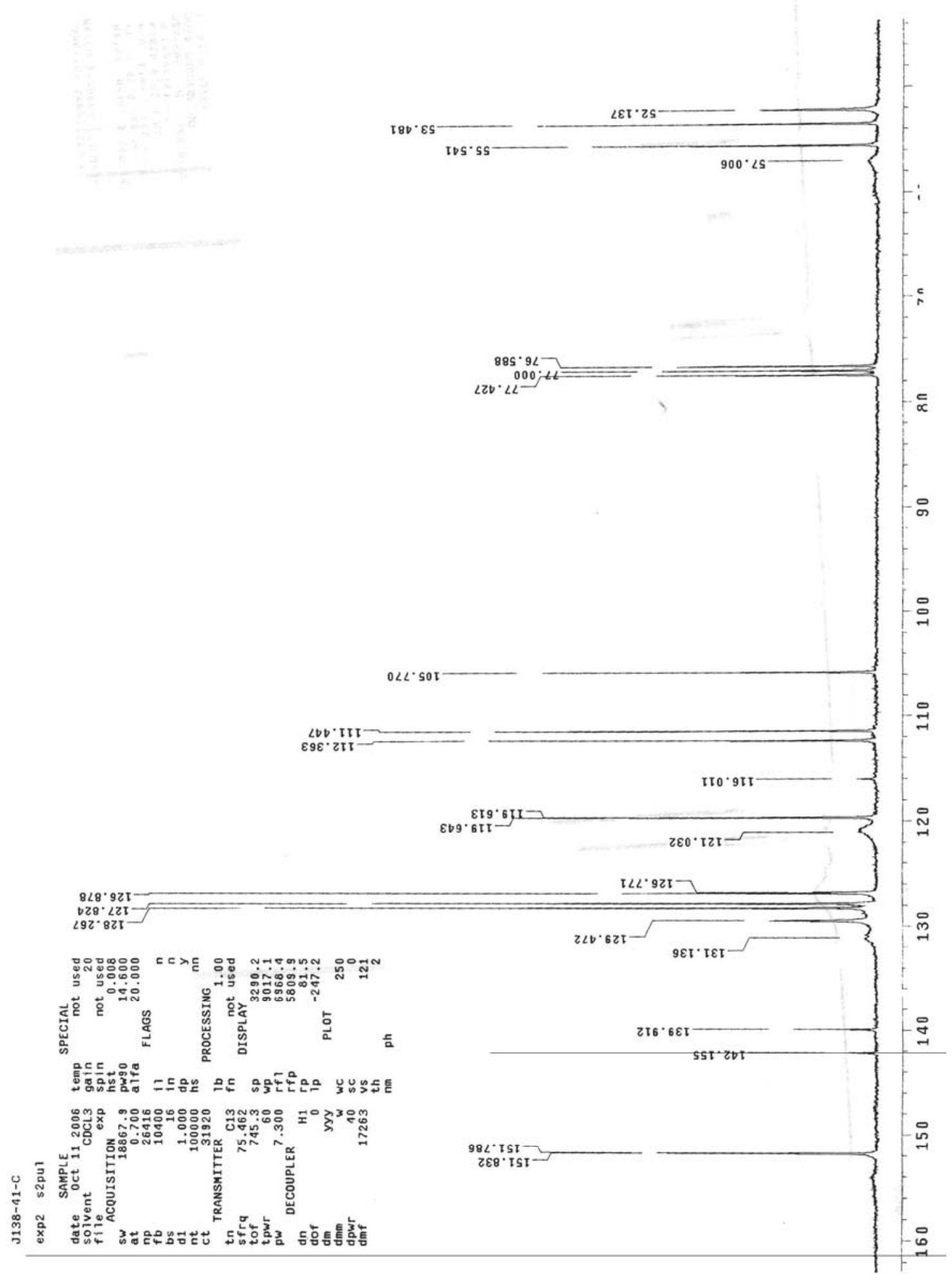

Fig. S-24: ${ }^{13} \mathrm{C}$ Spectra of 1-chloro-5,6-dihydro-6-[(benzylamino)methyl]-5-(2methoxylphenyl)pyrrolo[1,2-f]pteridine 5.6a. 

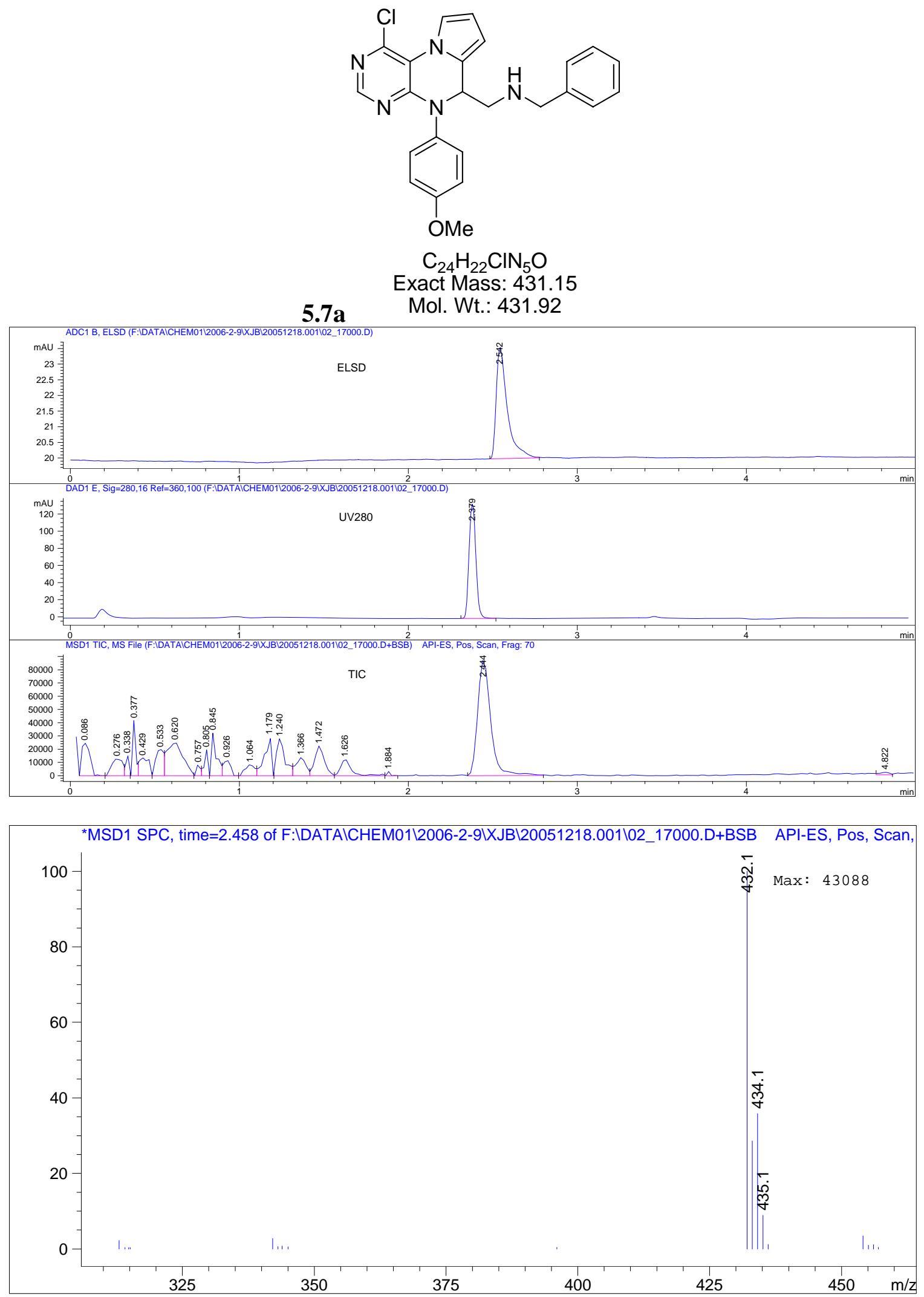

Fig. S-25: LC-MS-ELSD of 1-chloro-5,6-dihydro-6-[(benzylamino)methyl]-5-(4methoxylphenyl)pyrrolo[1,2-f]pteridine 5.7a. 

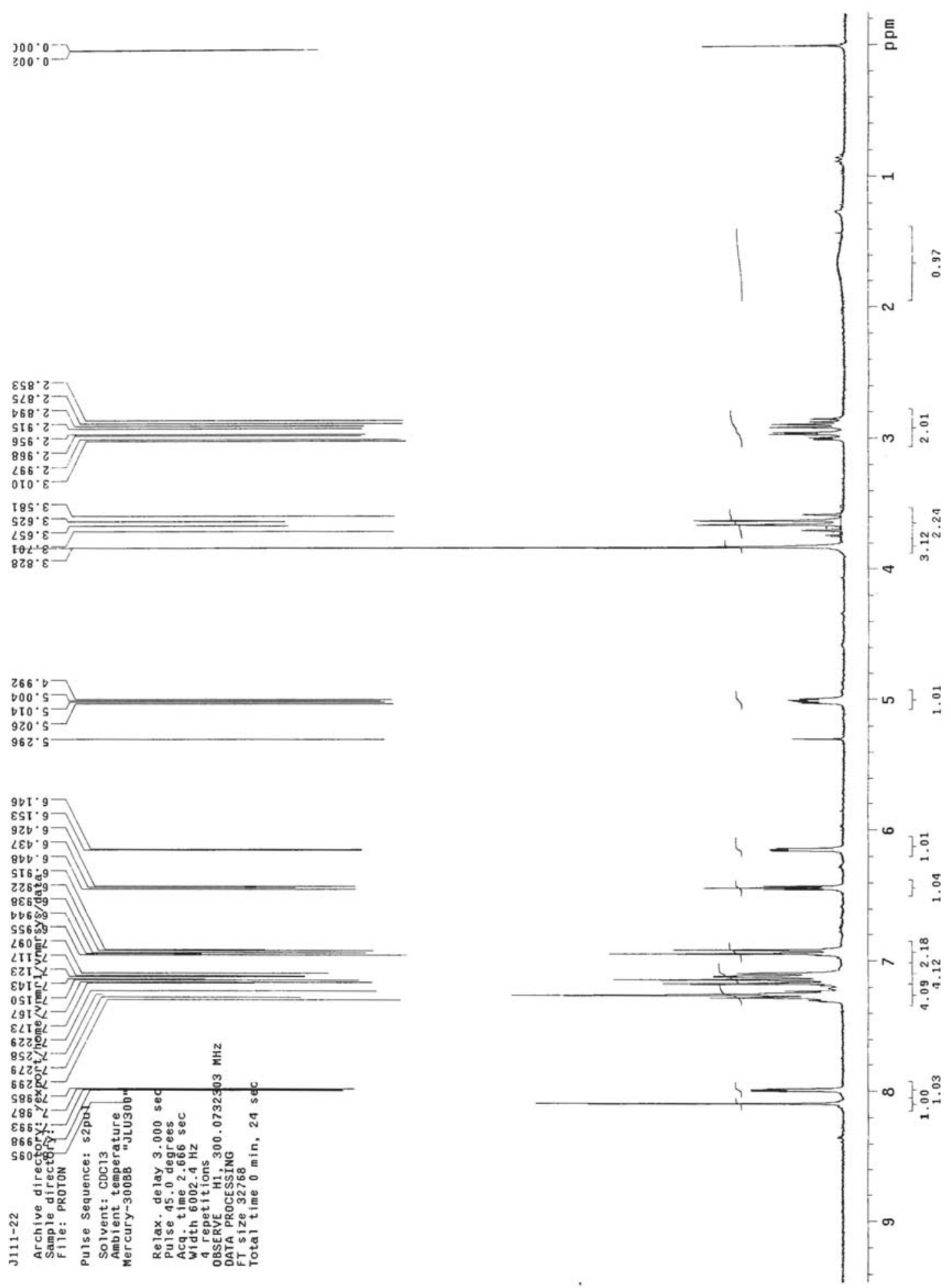

Fig. S-26: ${ }^{1} \mathrm{H}$ Spectra of 1-chloro-5,6-dihydro-6-[(benzylamino)methyl]-5-(4methoxylphenyl)pyrrolo[1,2-f]pteridine 5.7a. 


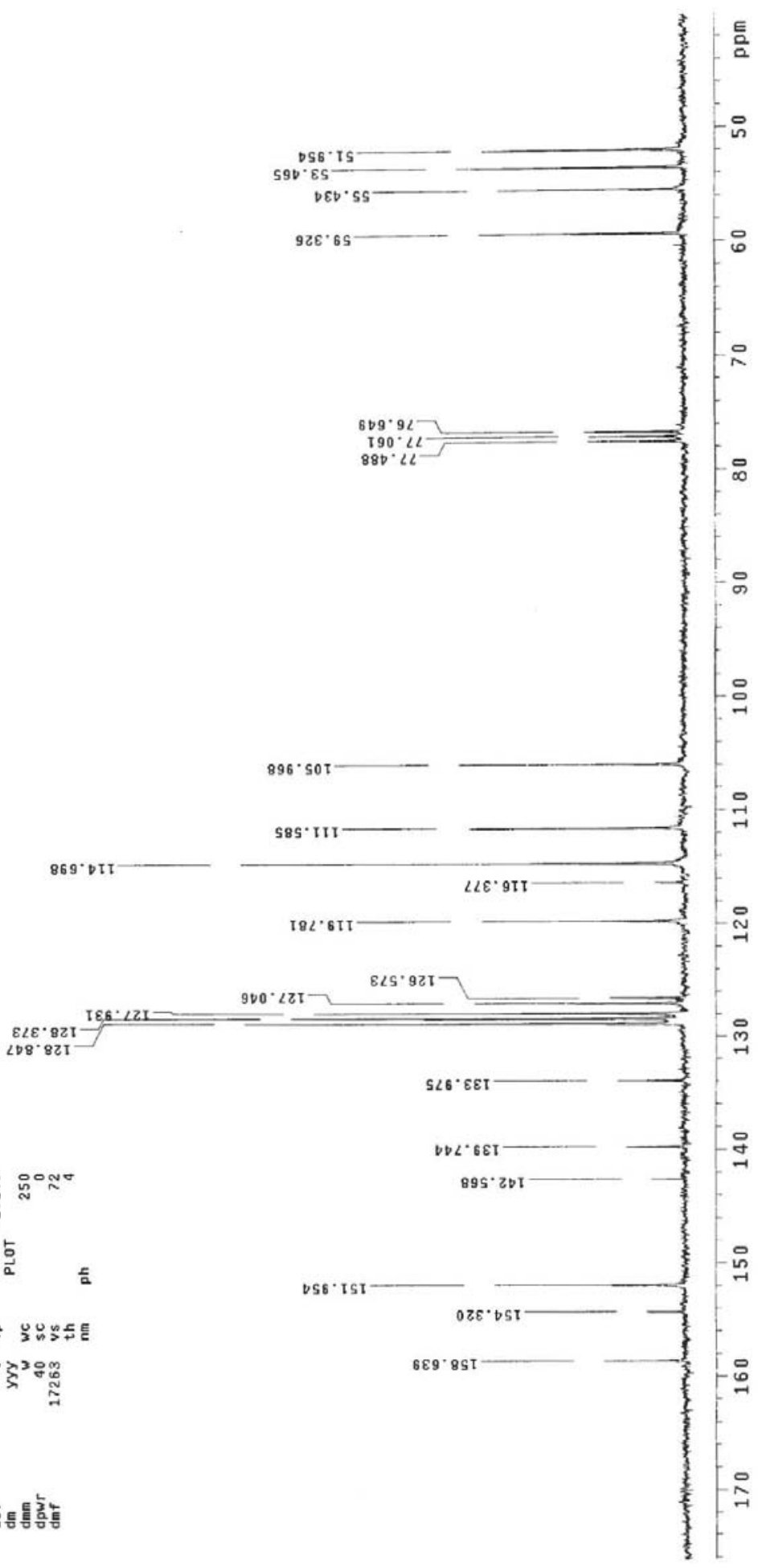

Fig. S-27: ${ }^{13} \mathrm{C}$ Spectra of 1-chloro-5,6-dihydro-6-[(benzylamino)methyl]-5-(4methoxylphenyl)pyrrolo[1,2-f]pteridine 5.7a. 


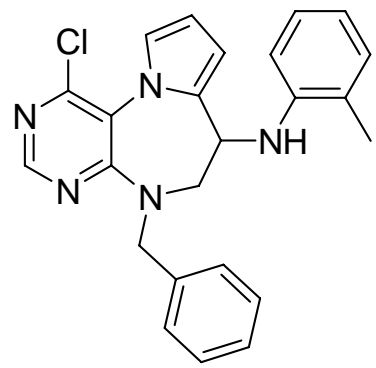

$\mathrm{C}_{24} \mathrm{H}_{22} \mathrm{ClN}_{5}$

Exact Mass: 415.16

4.8a

Mol. Wt.: 415.92
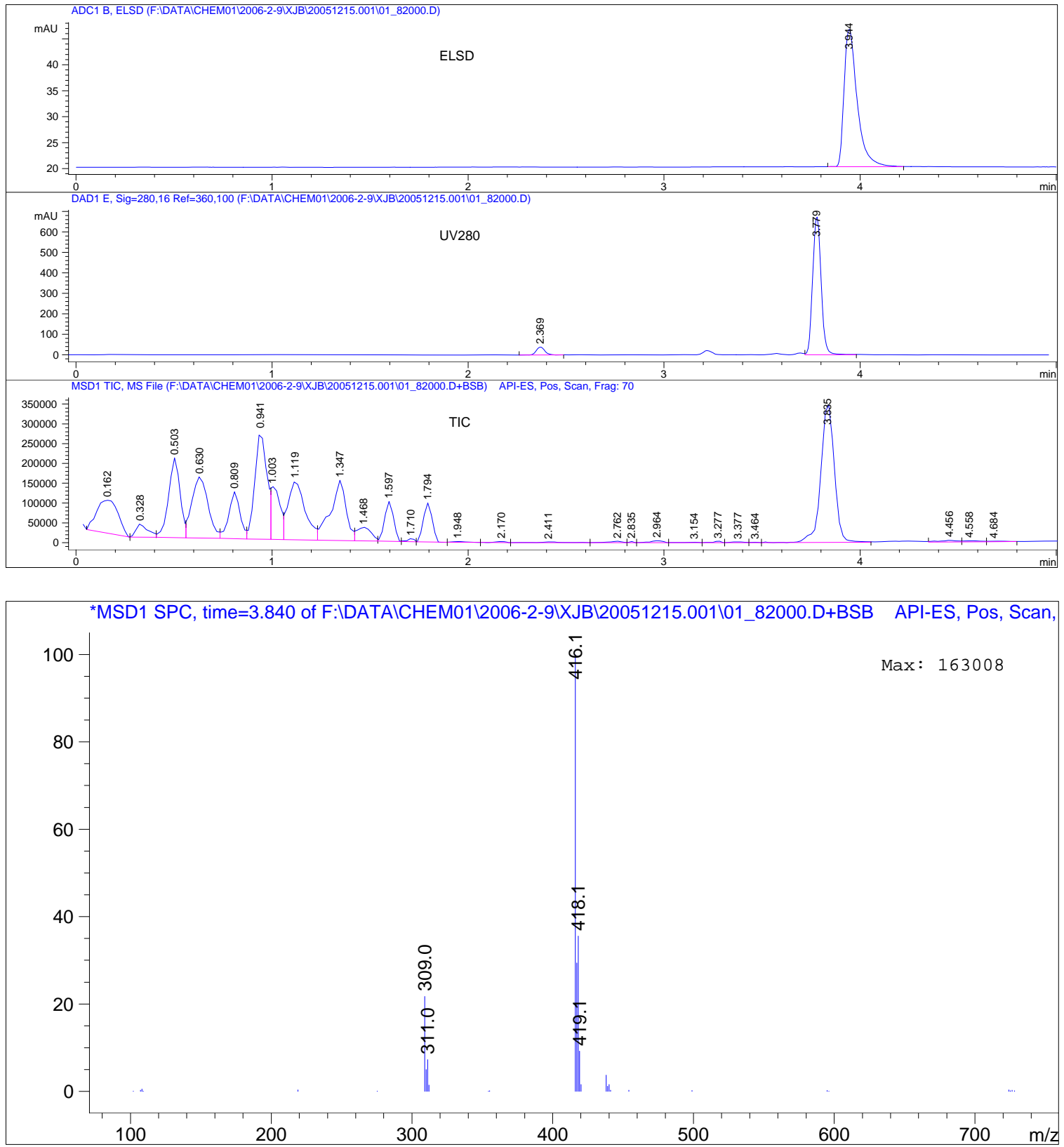

Fig. S-28: LC-MS-ELSD of 7-(2-methylphenylamino)-5-benzyl-1-chloro-6,7-dihydro pyrrolo[2,1-d][1,5]pyrimidodiazepine 4.8a. 


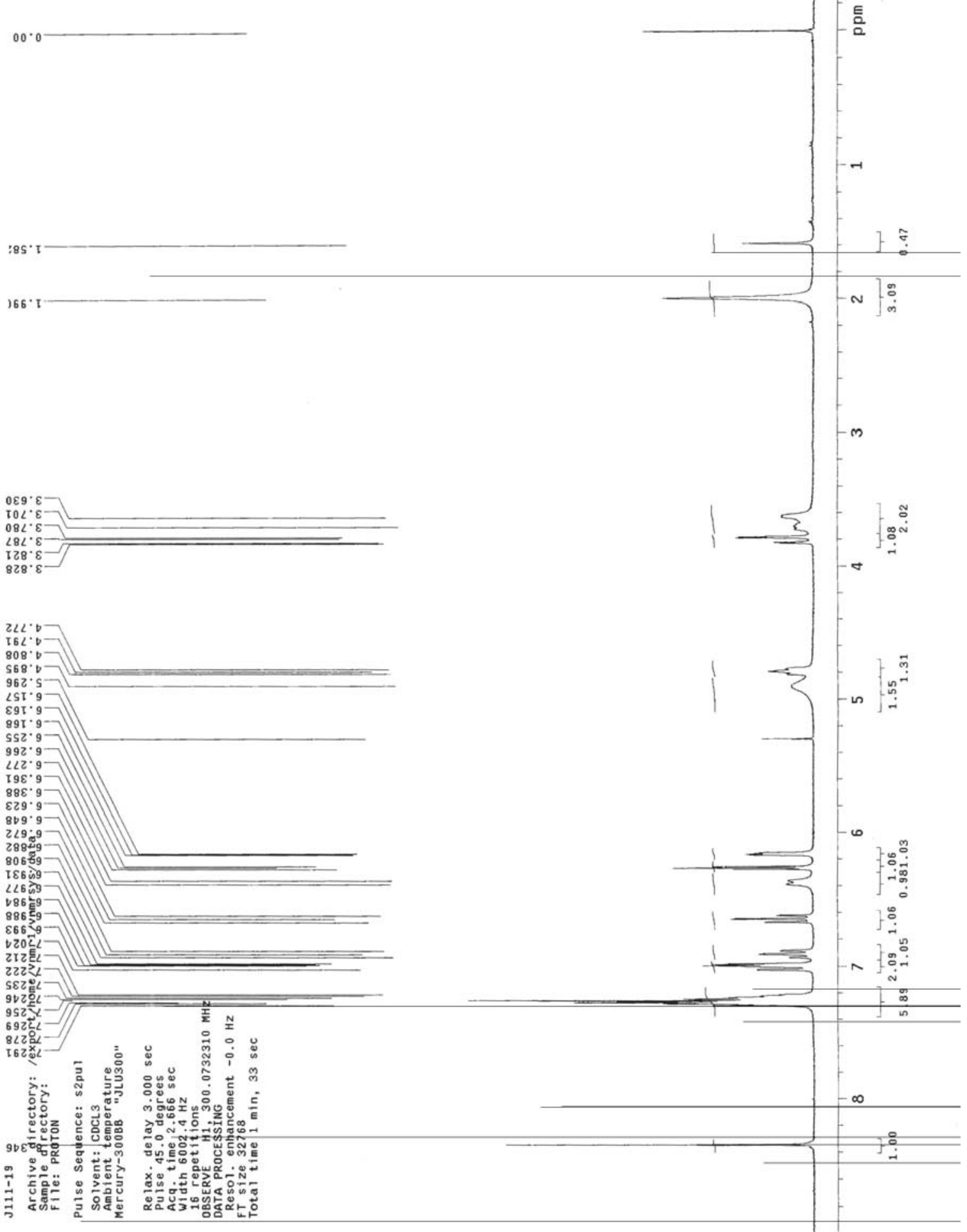

Fig. S-29: ${ }^{1} \mathrm{H}$ Spectra of 7-(2-methylphenylamino)-5-benzyl-1-chloro-6,7-dihydro pyrrolo[2,1-d][1,5]pyrimidodiazepine 4.8a. 


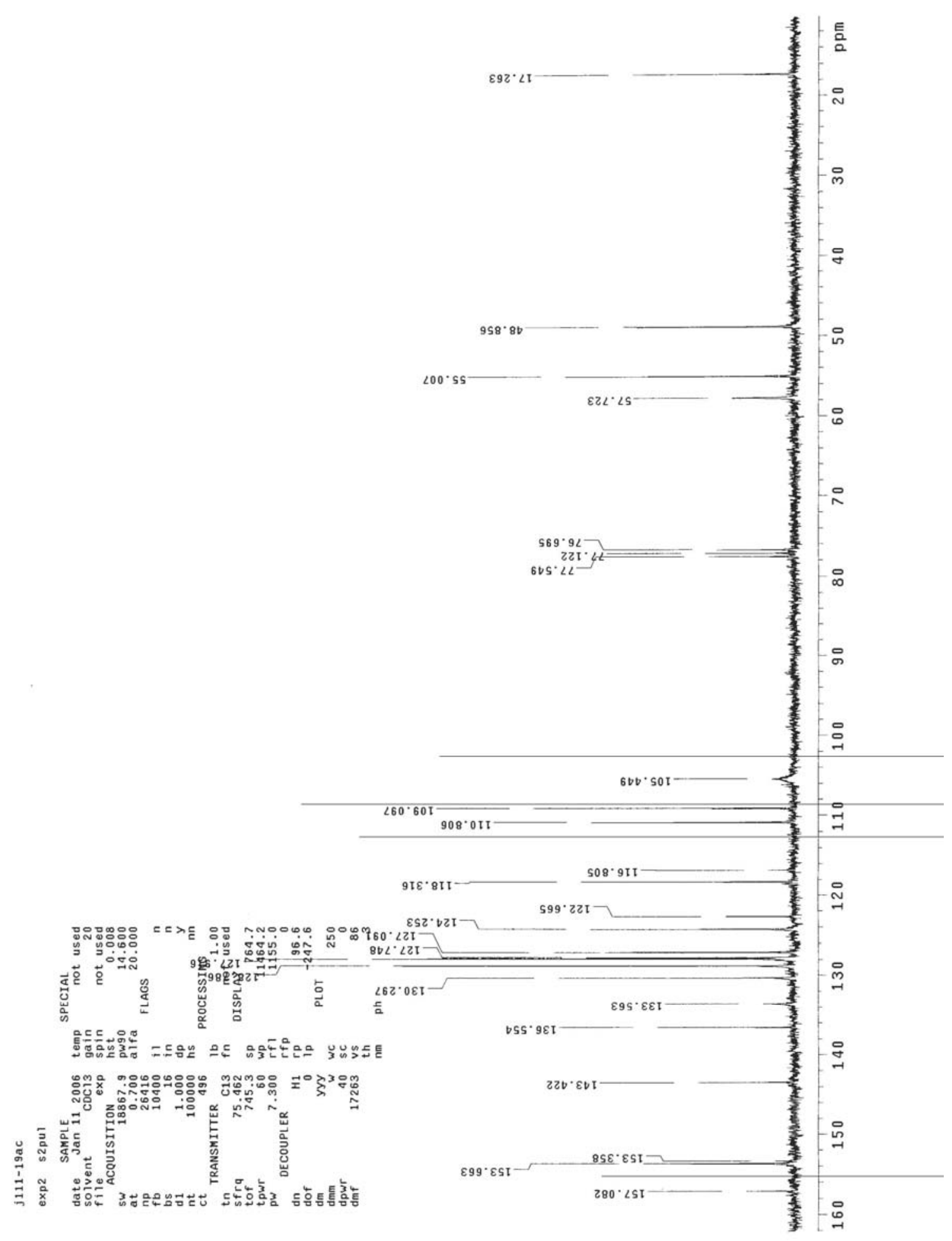

Fig. S-30: ${ }^{13} \mathrm{C}$ Spectra of 7-(2-methylphenylamino)-5-benzyl-1-chloro-6,7-dihydro pyrrolo[2,1-d][1,5]pyrimidodiazepine. 
<smiles>Cc1cccc(N2c3ncnc(Cl)c3-n3cccc3C2CNCc2ccccc2)c1</smiles>

$\mathrm{C}_{24} \mathrm{H}_{22} \mathrm{ClN}_{5}$

Exact Mass: 415.16

5.9a Mol. Wt.: 415.92
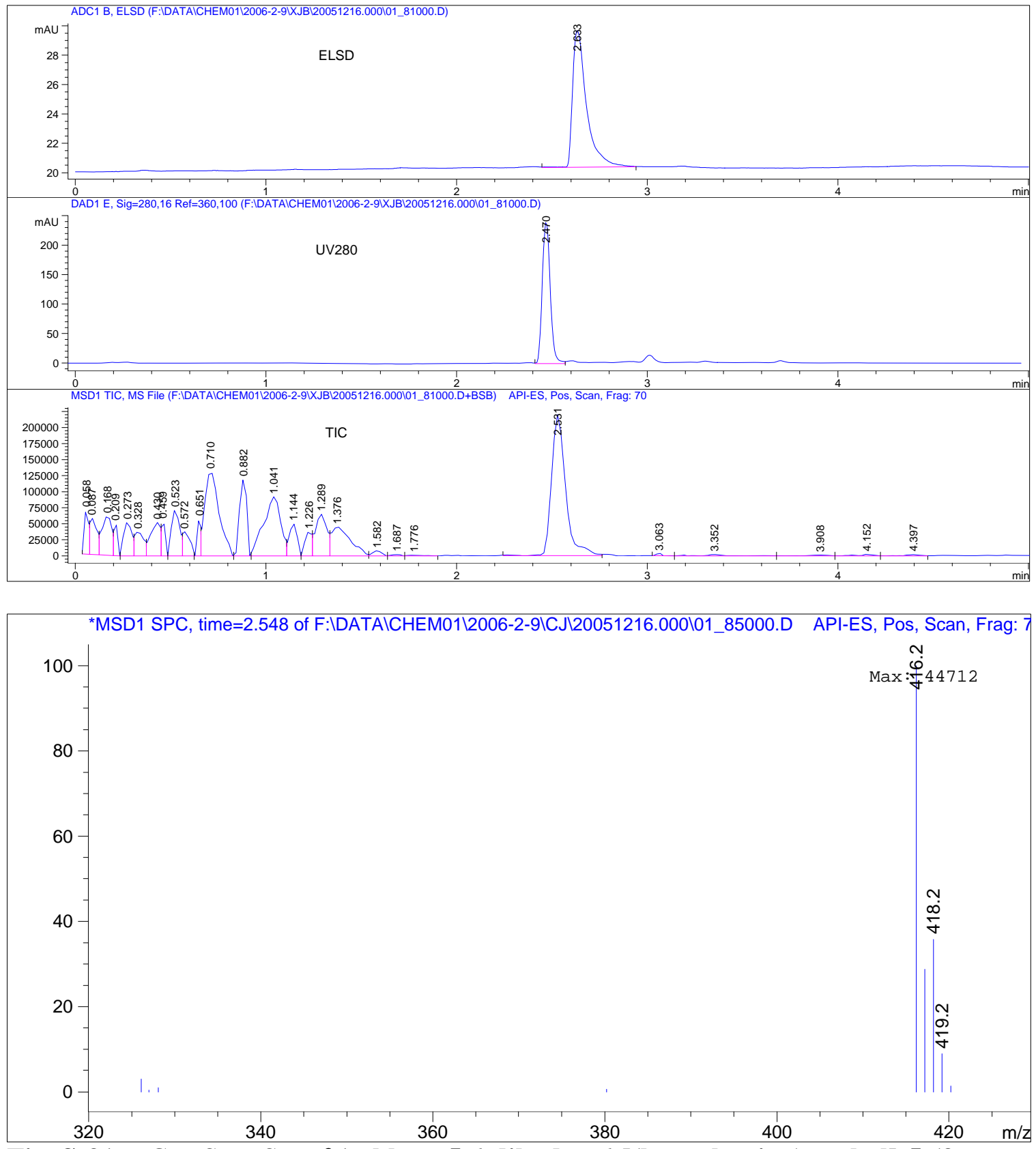

Fig. S-31: LC-MS-ELSD of 1-chloro-5,6-dihydro-6-[(benzylamino)methyl]-5-(3methylphenyl)pyrrolo[1,2-f]pteridine 5.9a. 


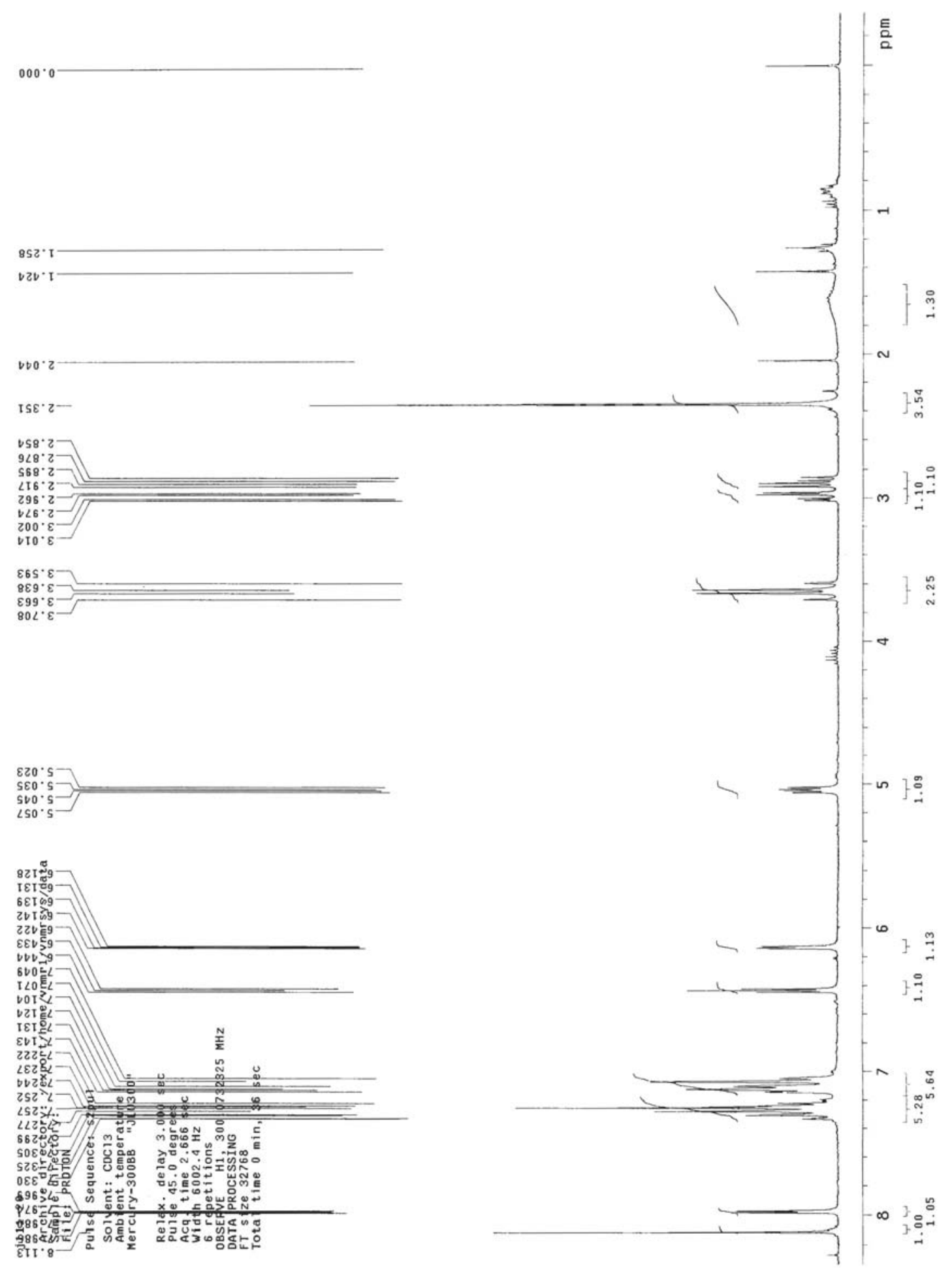

Fig. S-32: ${ }^{1} \mathrm{H}$ Spectra of 1-chloro-5,6-dihydro-6-[(benzylamino)methyl]-5-(3-methyl phenyl)pyrrolo[1,2-f]pteridine 5.9a. 


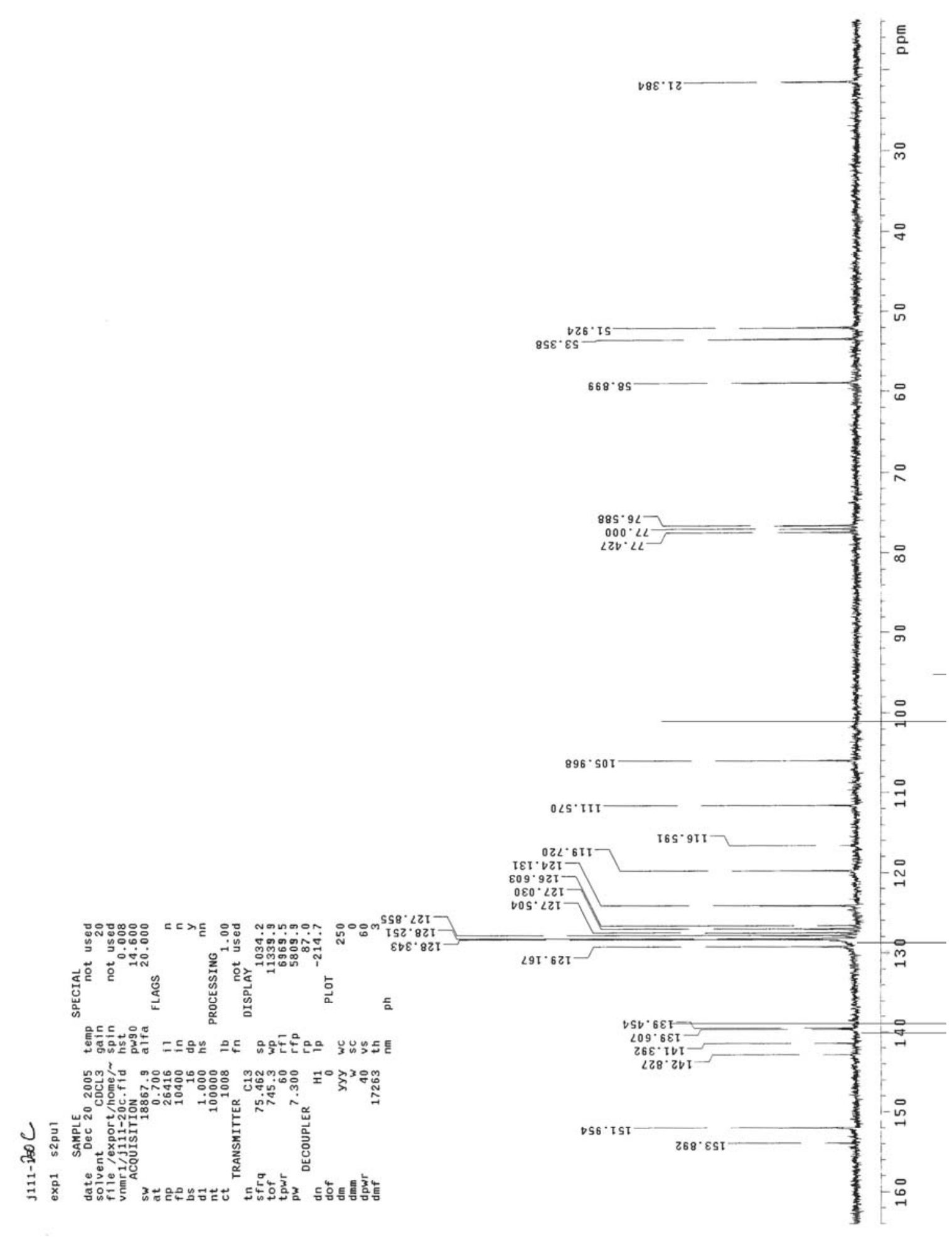

Fig.S-33: ${ }^{13} \mathrm{C}$ Spectra of 1-chloro-5,6-dihydro-6-[(benzylamino)methyl]-5-(3-methyl phenyl)pyrrolo[1,2-f]pteridine 5.9a. 


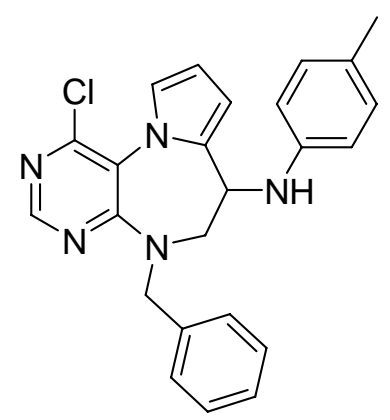

$\mathrm{C}_{24} \mathrm{H}_{22} \mathrm{ClN}_{5}$

Exact Mass: 415.16

4.10a

Mol. Wt.: 415.92
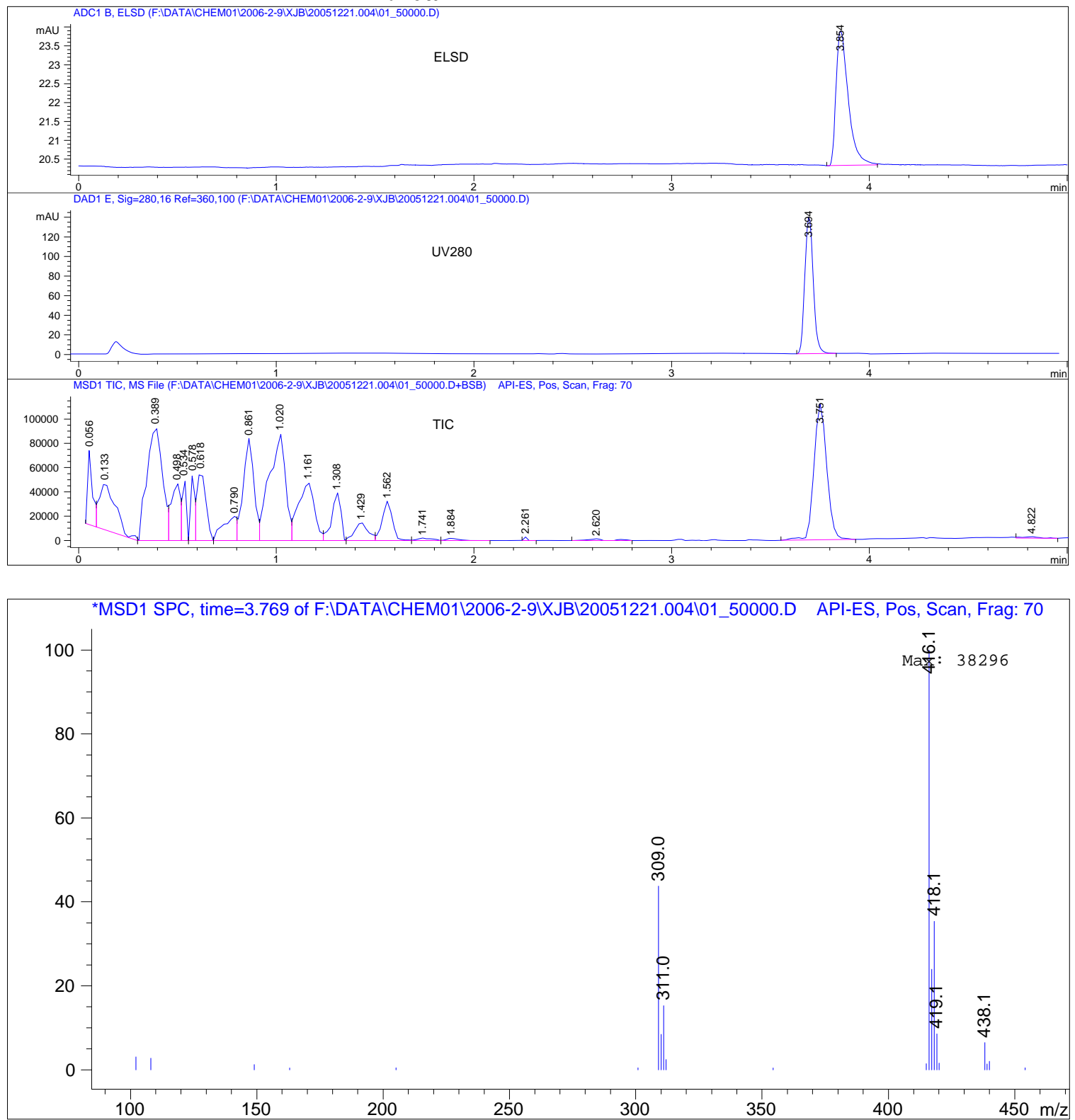

Fig. S-34: LC-MS-ELSD of 7-(4-methylphenylamino)-5-benzyl-1-chloro-6,7-dihydro pyrrolo[2,1-d][1,5]pyrimidodiazepine 4.10a. 


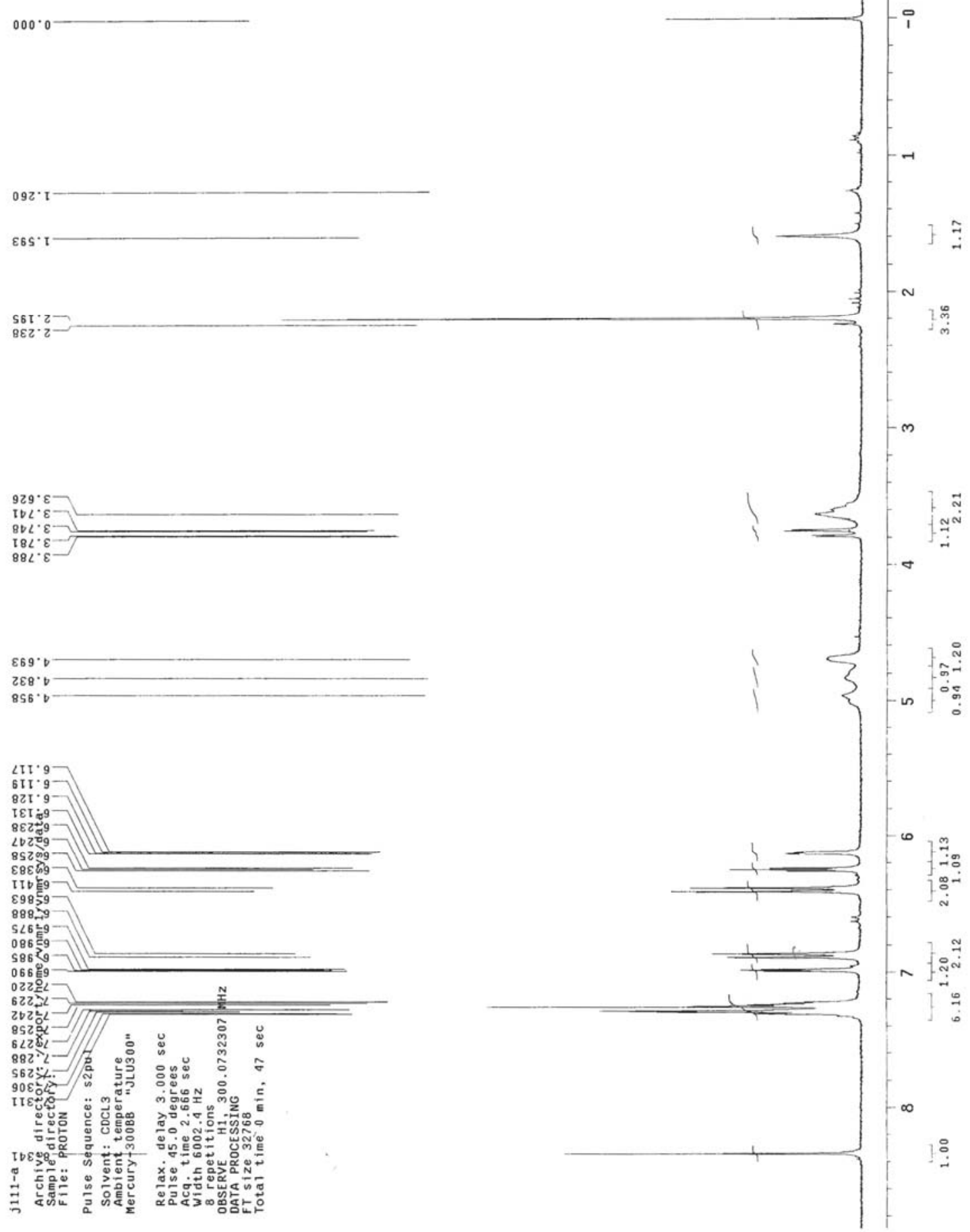

Fig. S-35: ${ }^{1} \mathrm{H}$ Spectra of 7-(4-methylphenylamino)-5-benzyl-1-chloro-6,7-dihydro pyrrolo[2,1-d][1,5]pyrimidodiazepine 4.10a. 


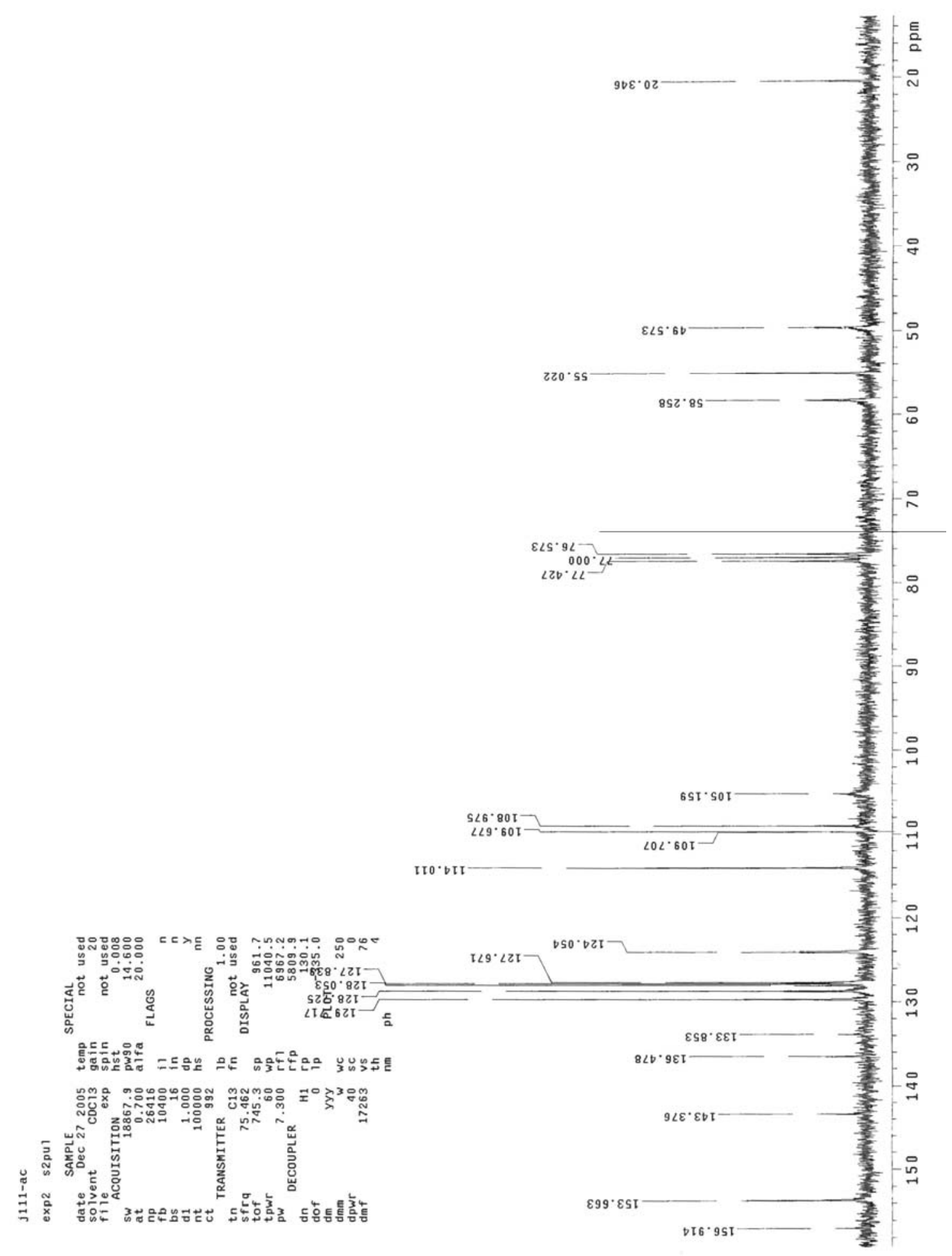

Fig. S-36: ${ }^{13} \mathrm{C}$ Spectra of 7-(4-methylphenylamino)-5-benzyl-1-chloro-6,7-dihydro pyrrolo[2,1-d][1,5]pyrimidodiazepine 4.10a. 


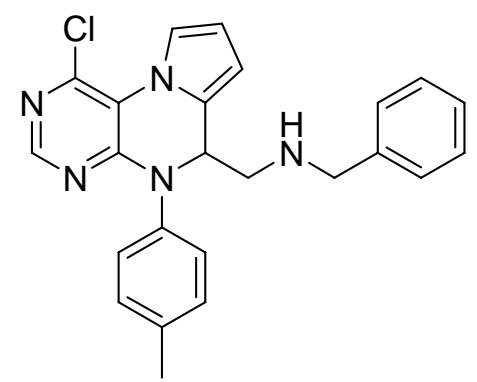

$\mathrm{C}_{24} \mathrm{H}_{22} \mathrm{ClN}_{5}$

Exact Mass: 415.16

$5.10 \mathrm{a}$

Mol. Wt.: 415.92
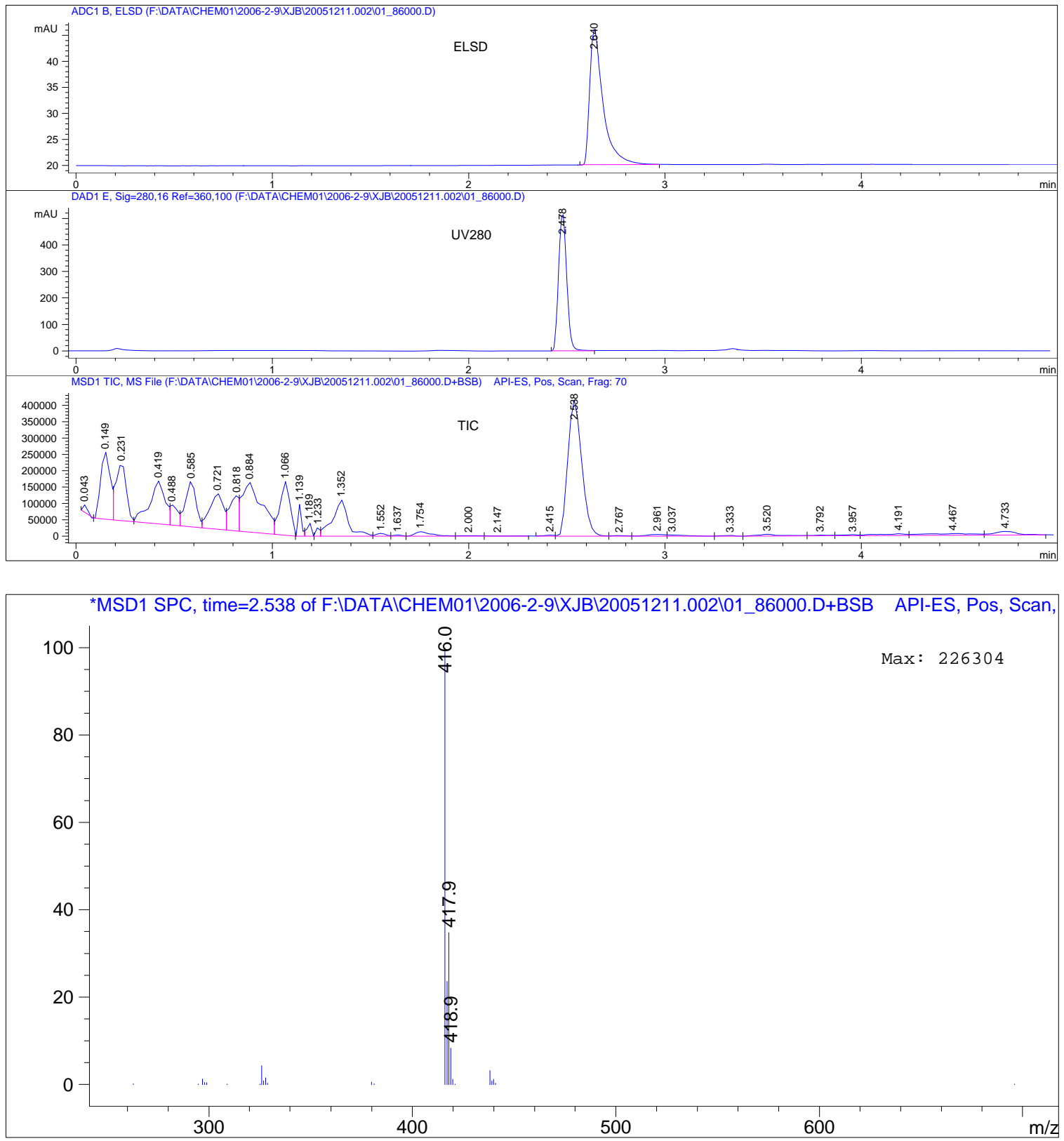

Fig. S-37: LC-MS-ELSD of 1-chloro-5,6-dihydro-6-[(benzylamino)methyl]-5-(4methylphenyl)pyrrolo[1,2-f]pteridine 5.10a. 


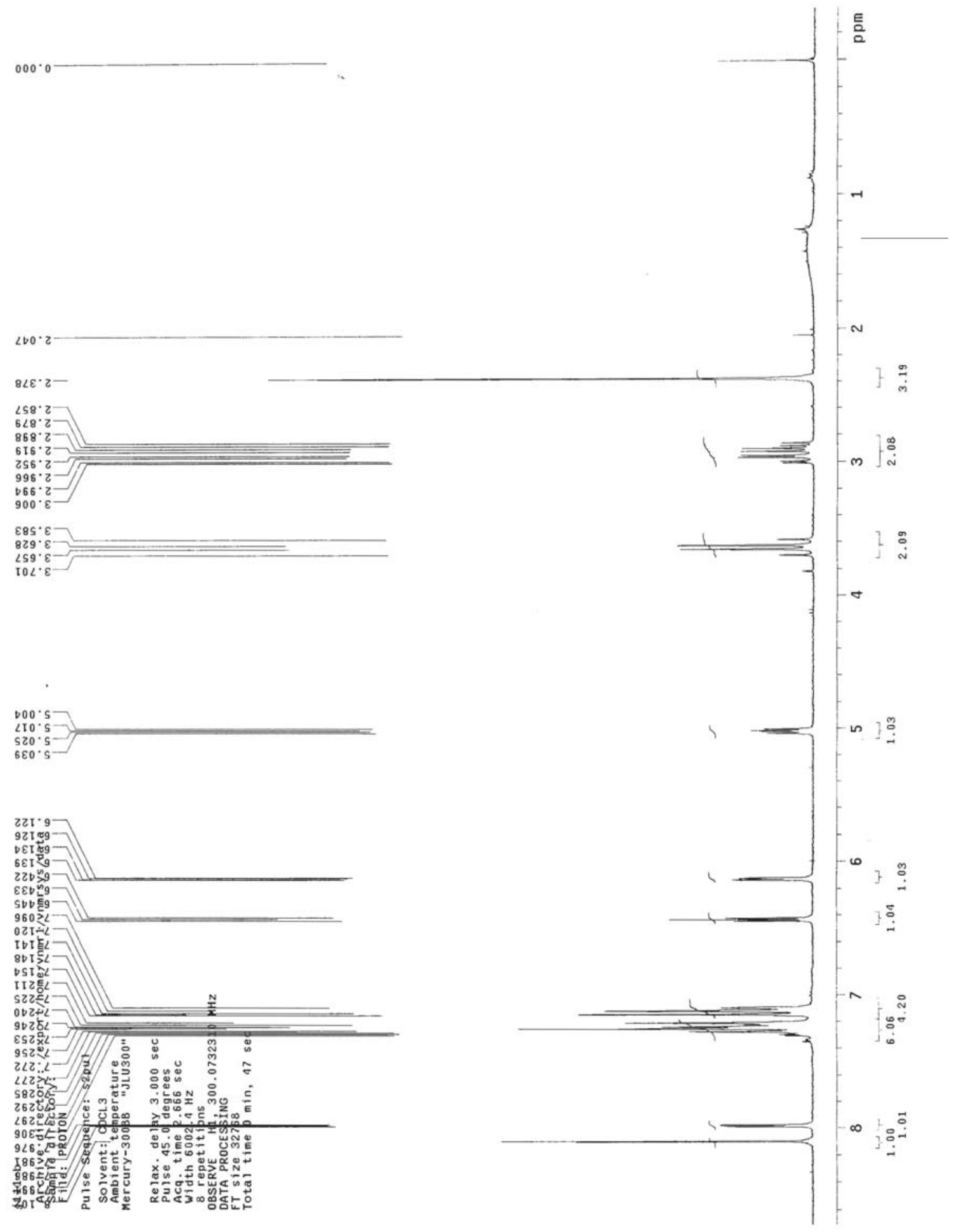

Fig. S-38: ${ }^{1} \mathrm{H}$ Spectra of 1-chloro-5,6-dihydro-6-[(benzylamino)methyl]-5-(4-methyl phenyl)pyrrolo[1,2-f]pteridine 5.10a. 


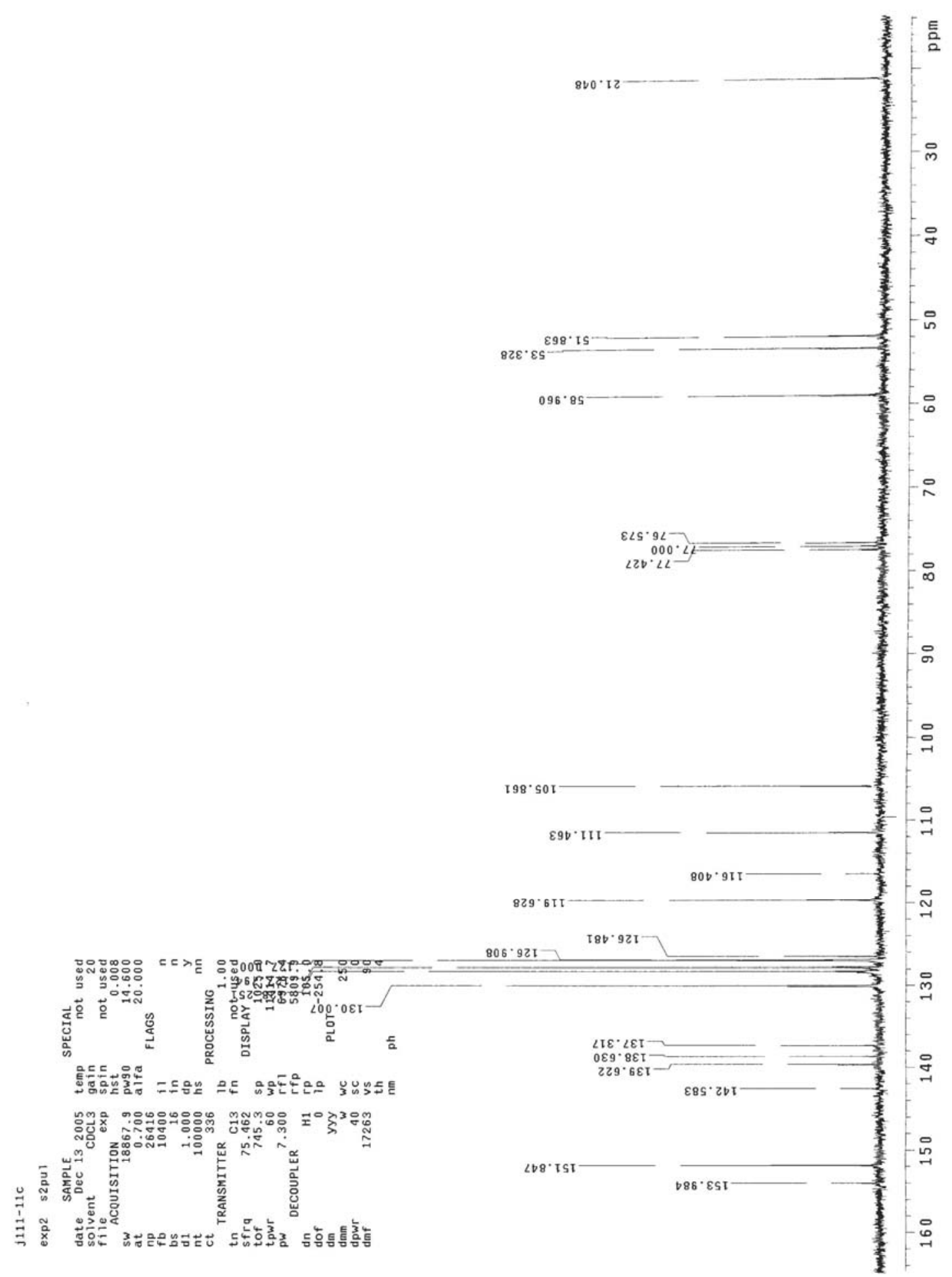

Fig. S-39: ${ }^{13} \mathrm{C}$ Spectra of 1-chloro-5,6-dihydro-6-[(benzylamino)methyl]-5-(4-methyl phenyl)pyrrolo[1,2-f]pteridine 5.10a. 

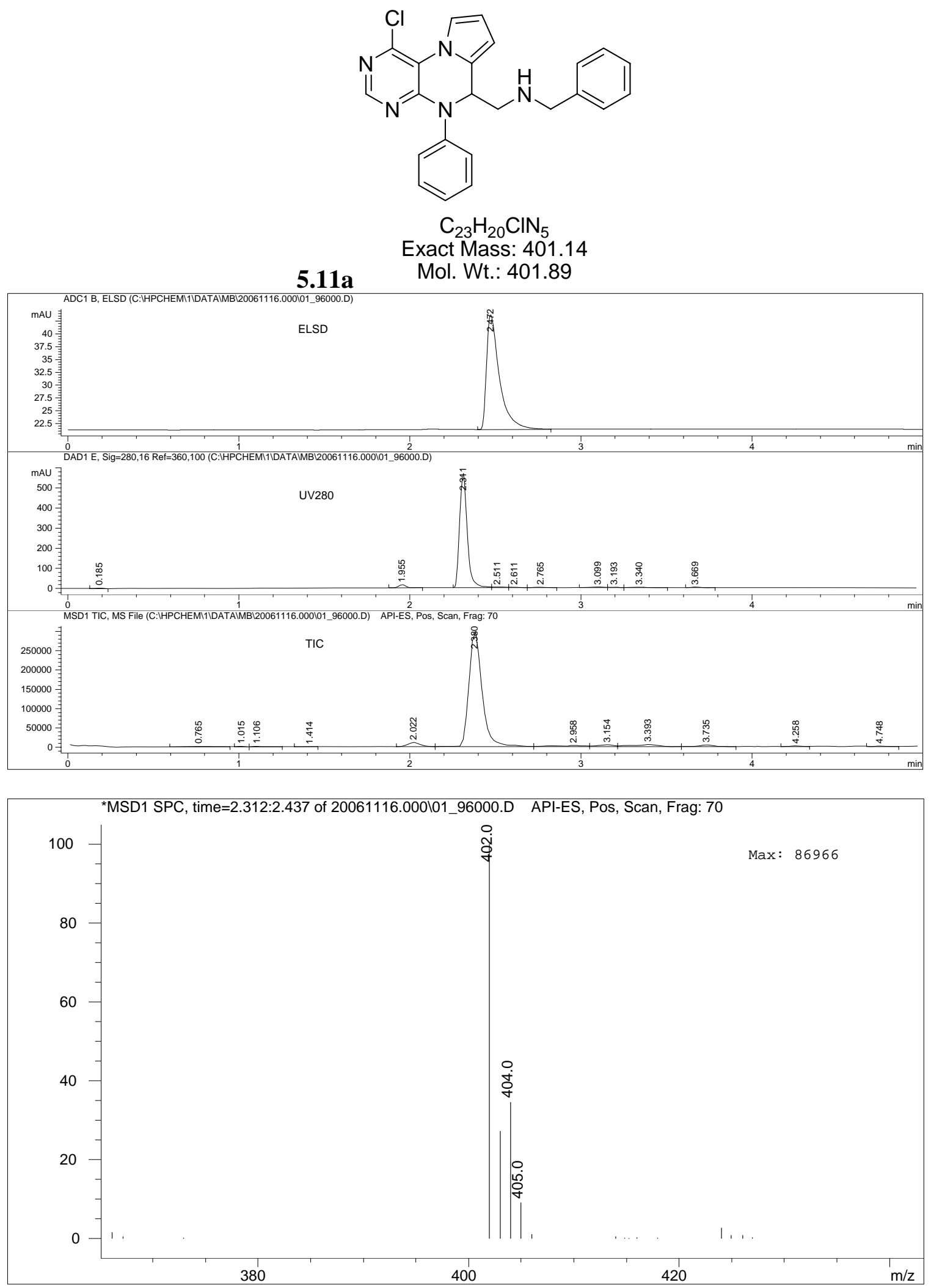

Fig. S-40: LC-MS-ELSD of 1-chloro-5,6-dihydro-6-[(benzylamino)methyl]-5-phenyl pyrrolo[1,2-f]pteridine 5.11a. 


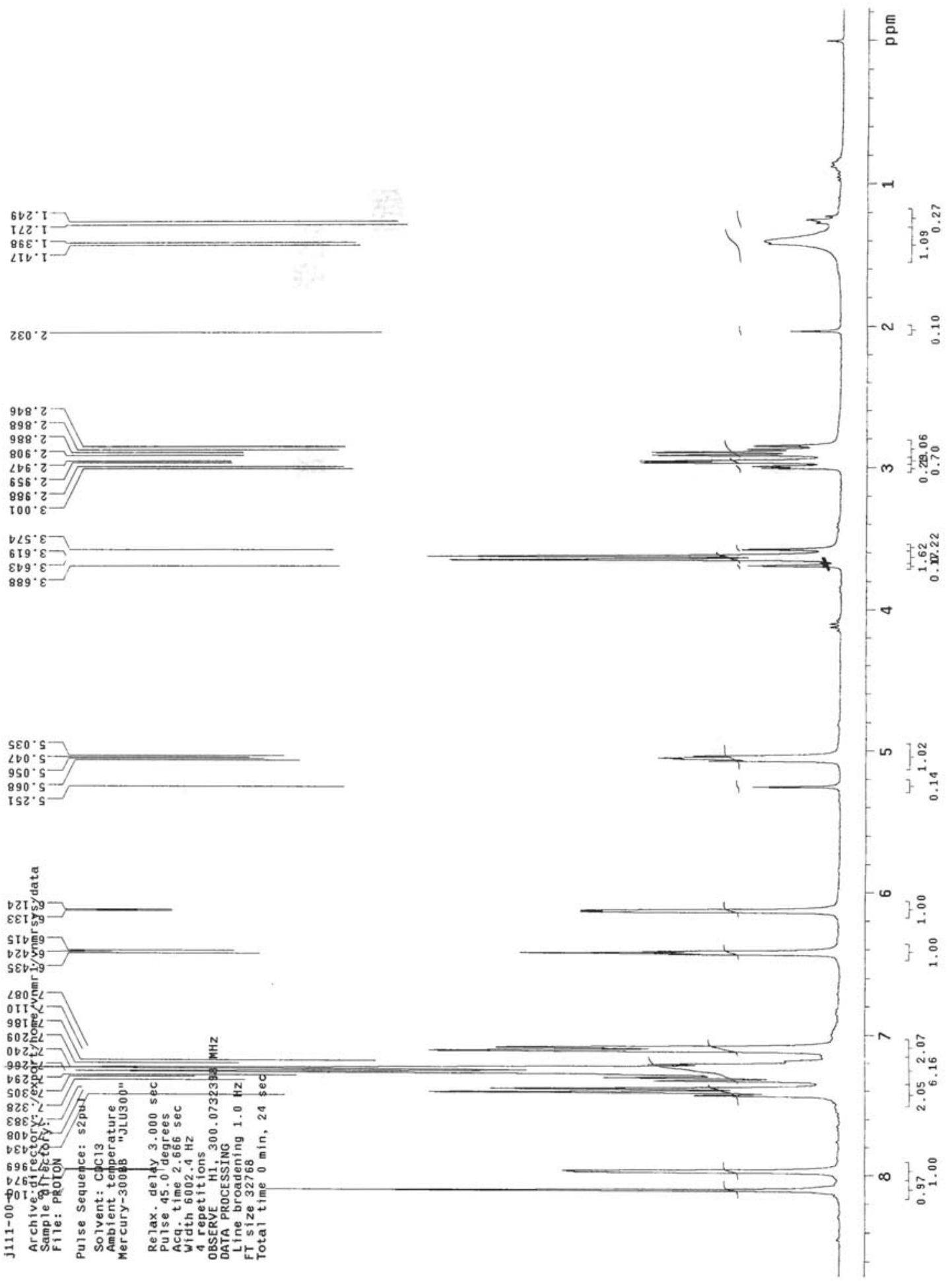

Fig. S-41: ${ }^{1} \mathrm{H}$ Spectra of 1-chloro-5,6-dihydro-6-[(benzylamino)methyl]-5-phenyl pyrrolo[1,2-f]pteridine 5.11a. 


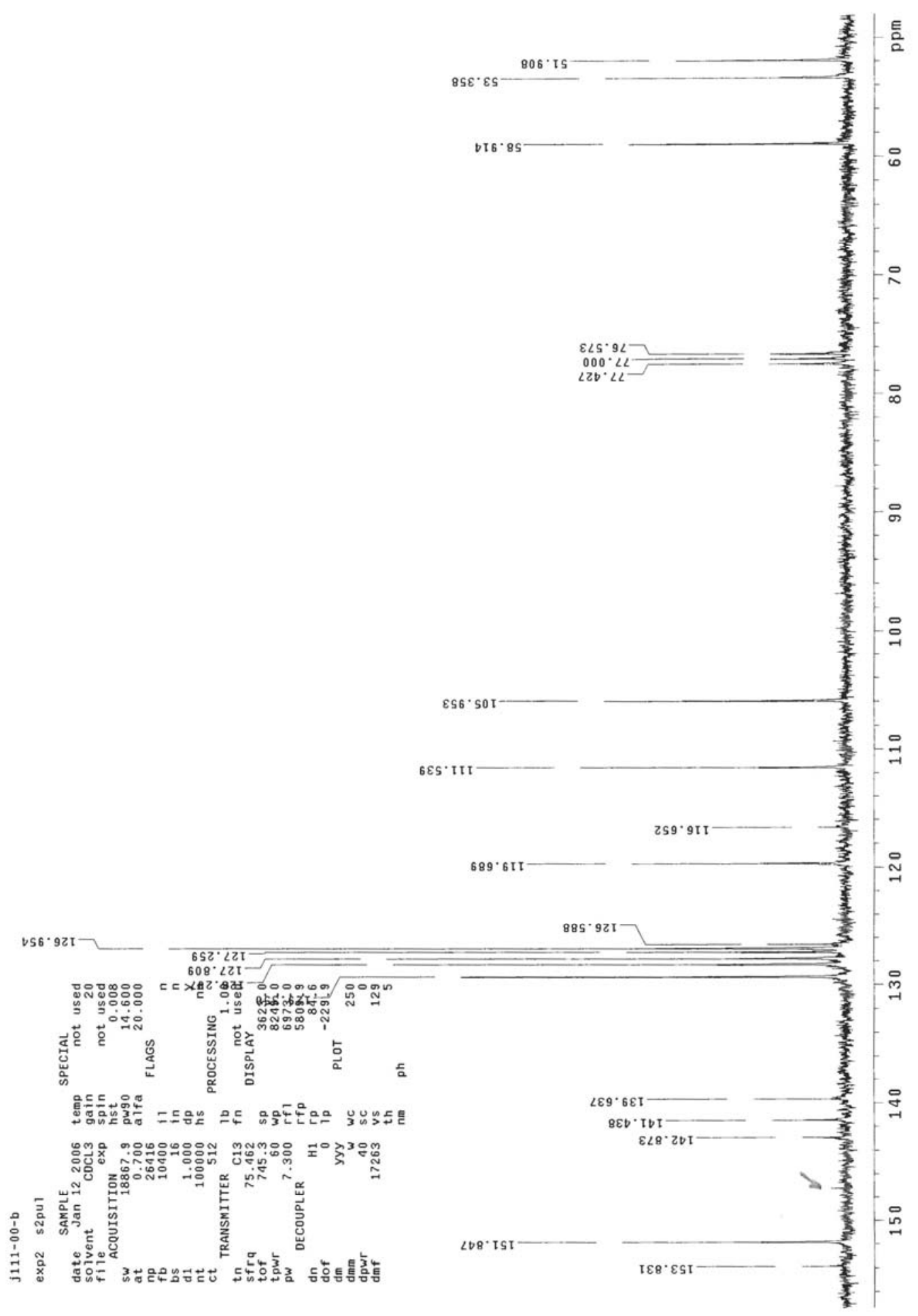

Fig. S-42: ${ }^{13} \mathrm{C}$ Spectra of 1-chloro-5,6-dihydro-6-[(benzylamino)methyl]-5-phenyl pyrrolo[1,2-f]pteridine 5.11a. 


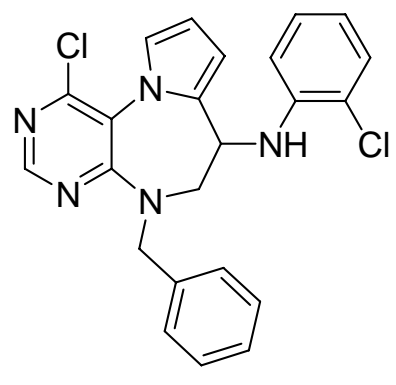

$\mathrm{C}_{23} \mathrm{H}_{19} \mathrm{Cl}_{2} \mathrm{~N}_{5}$

Exact Mass: 435.1

4.12a

Mol. Wt.: 436.34
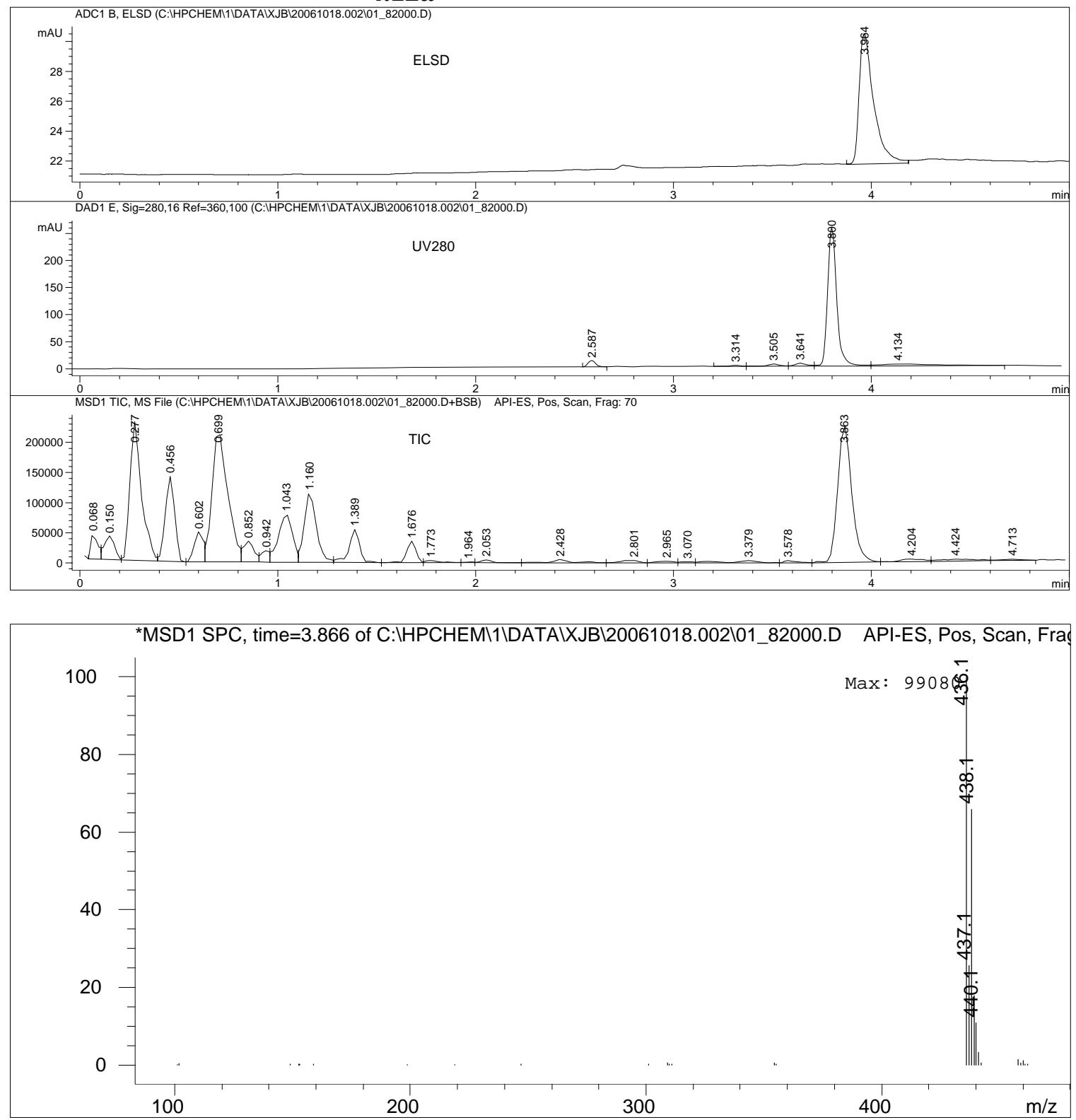

Fig. S-43: LC-MS-ELSD of 7-(2-chlorophenylamino)-5-benzyl-1-chloro-6,7-dihydro pyrrolo[2,1-d][1,5]pyrimidodiazepine 4.12a. 


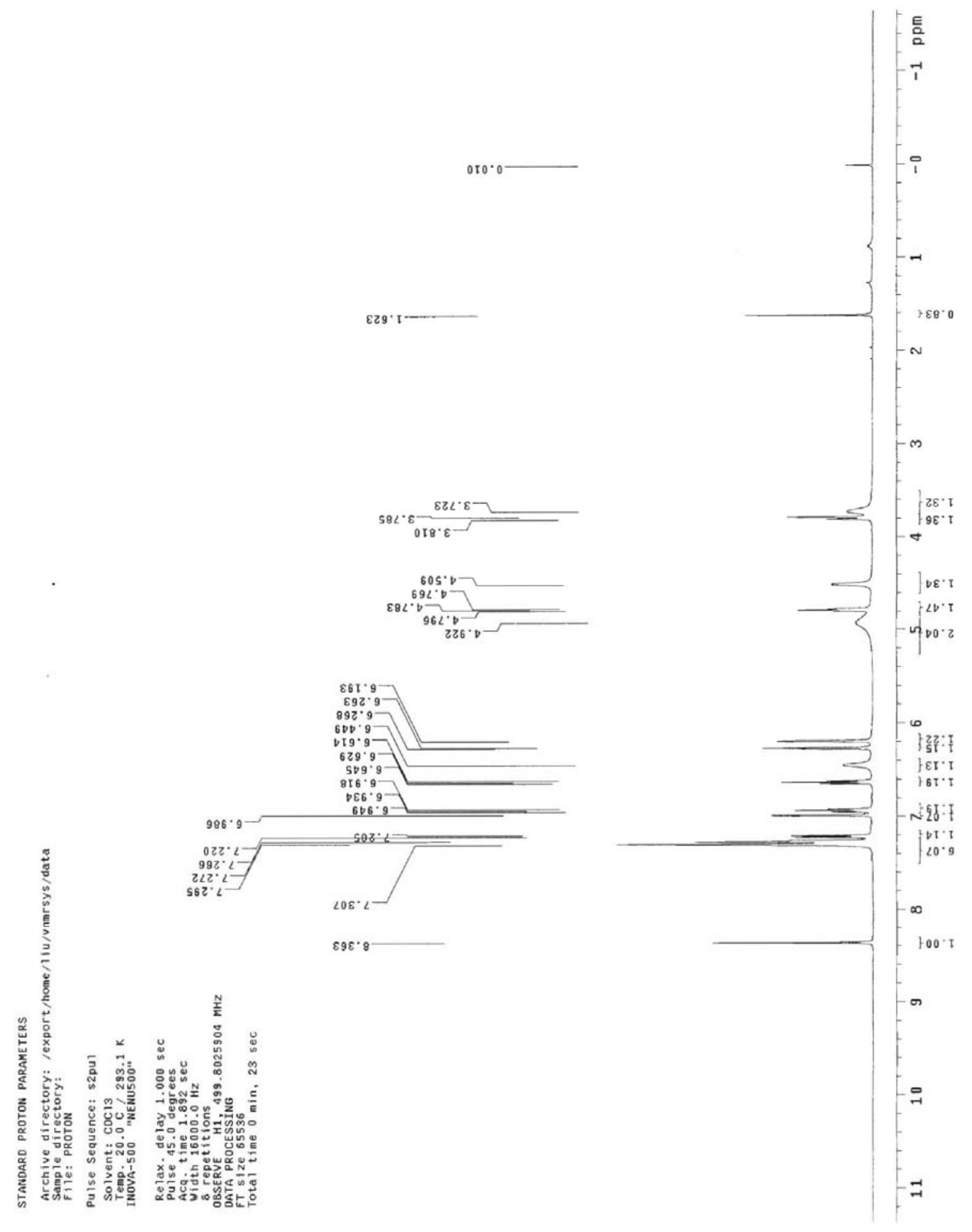

Fig. S-44: ${ }^{1} \mathrm{H}$ Spectra of 7-(2-chlorophenylamino)-5-benzyl-1-chloro-6,7-dihydro pyrrolo[2,1-d][1,5]pyrimidodiazepine 4.12a. 


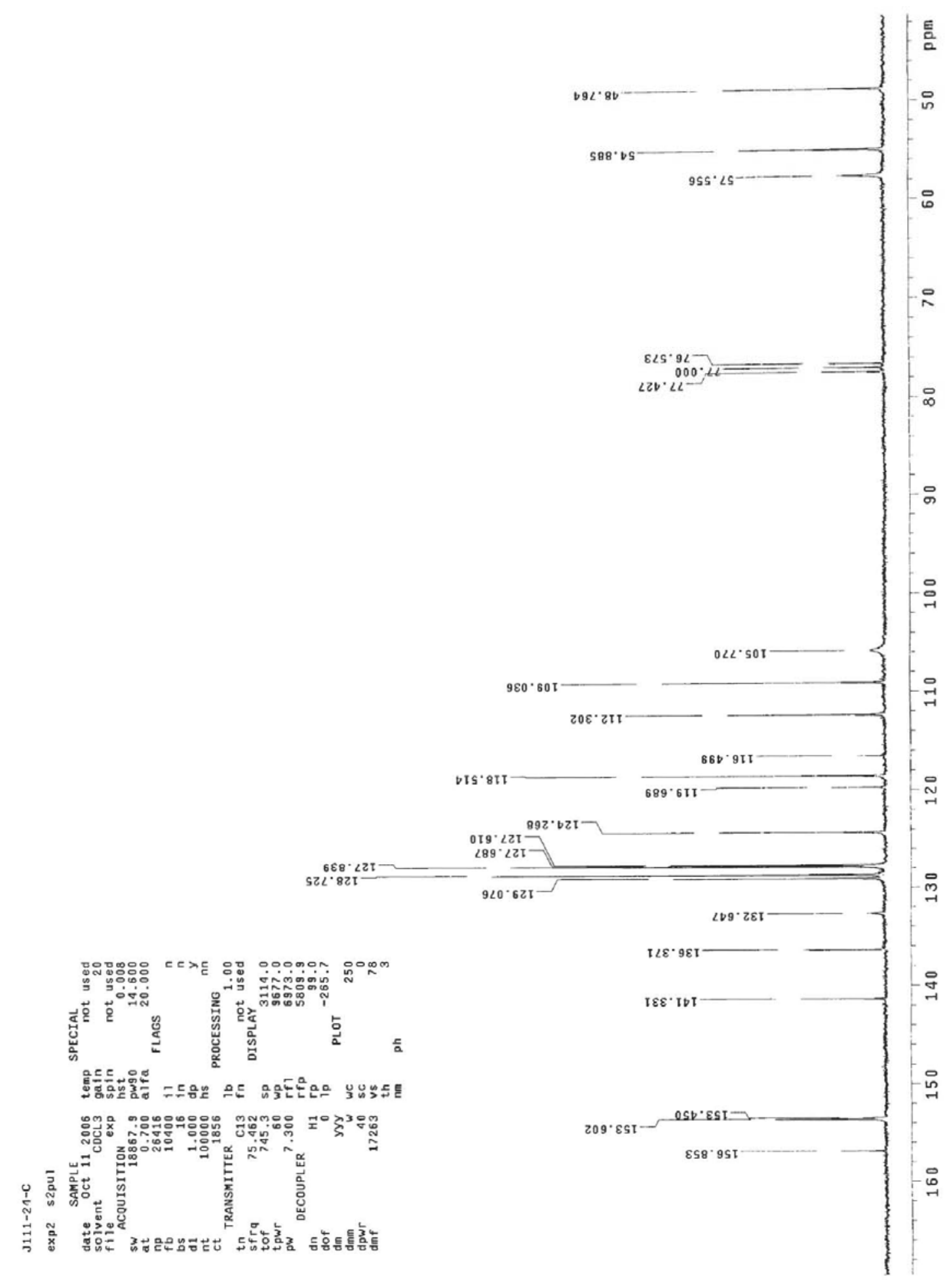

Fig. S-45: ${ }^{13} \mathrm{C}$ Spectra of 7-(2-chlorophenylamino)-5-benzyl-1-chloro-6,7-dihydro pyrrolo[2,1-d][1,5]pyrimidodiazepine 4.12a. 


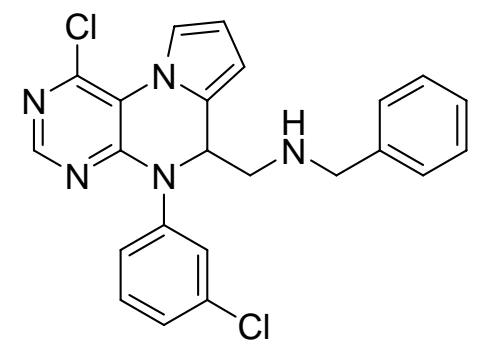

$$
\mathrm{C}_{23} \mathrm{H}_{19} \mathrm{Cl}_{2} \mathrm{~N}_{5}
$$

Exact Mass: 435.1

$5.13 a$

Mol. Wt.: 436.34
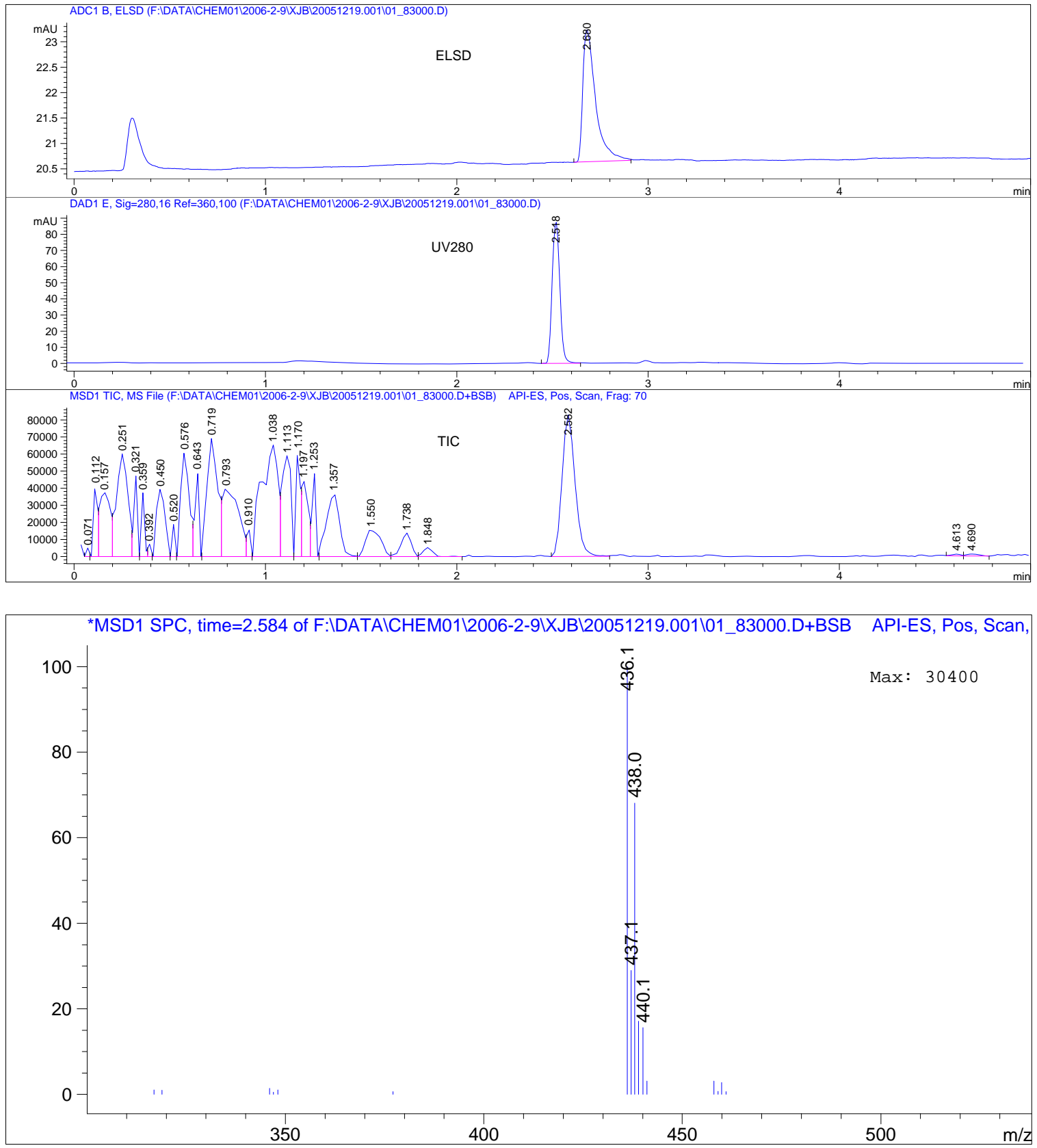

Fig. S-46: LC-MS-ELSD of 1-chloro-5,6-dihydro-6-[(benzylamino)methyl]-5-(3chlorophenyl)pyrrolo[1,2-f]pteridine 5.13a. 

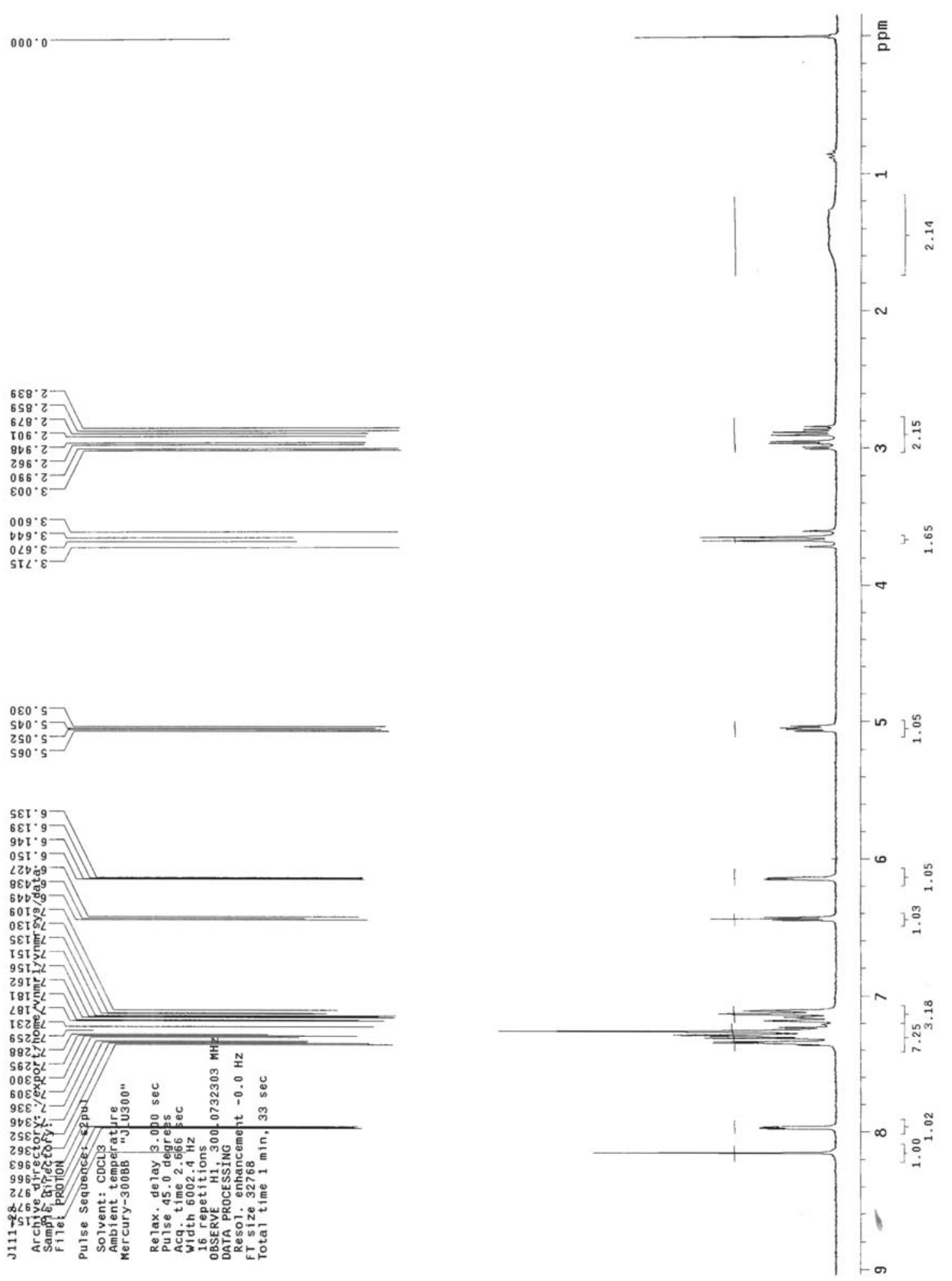

Fig. S-47: ${ }^{1} \mathrm{H}$ Spectra of 1-chloro-5,6-dihydro-6-[(benzylamino)methyl]-5-(3-chloro phenyl)pyrrolo[1,2-f]pteridine 5.13a. 

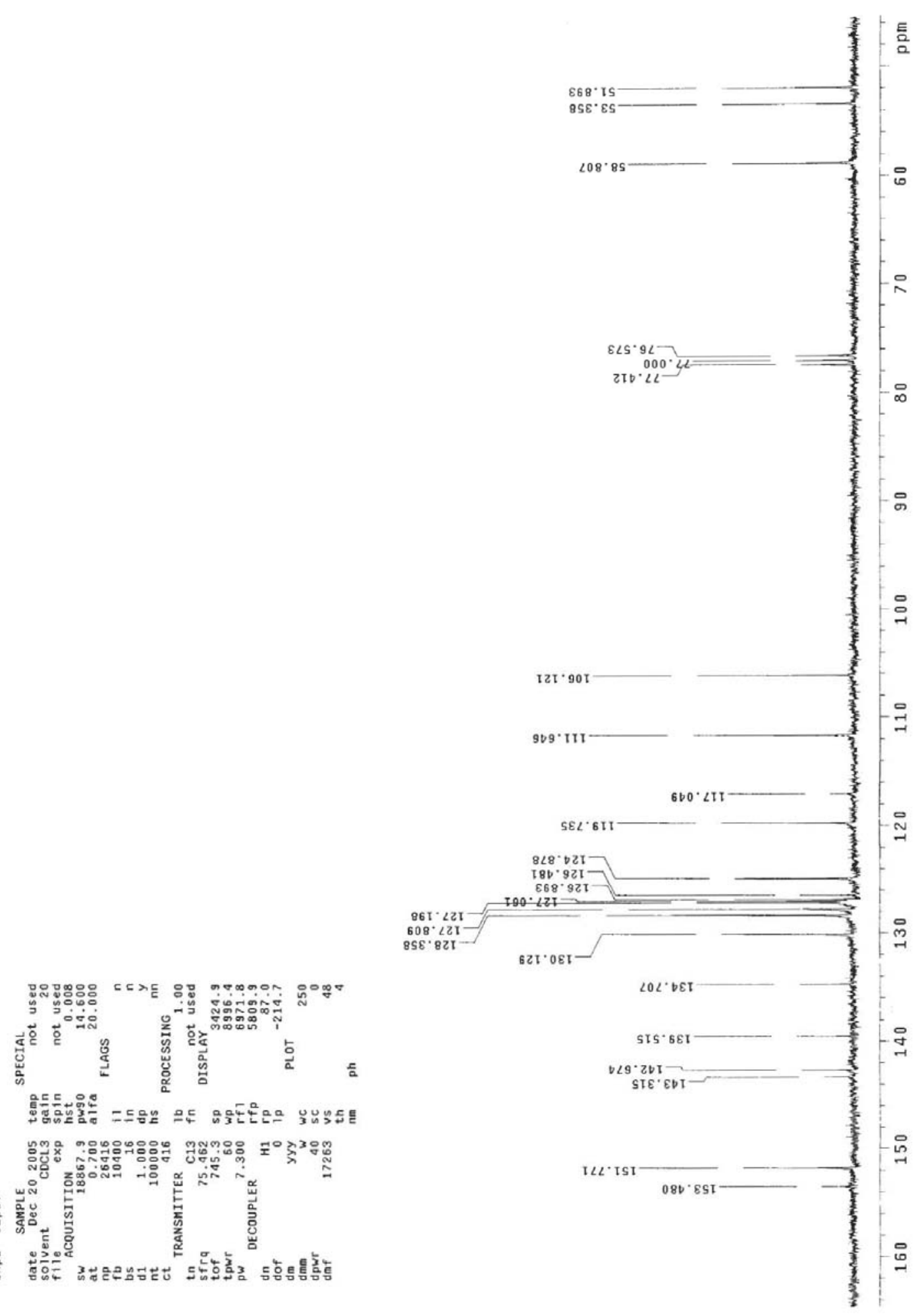

Fig. S-48: ${ }^{13} \mathrm{C}$ Spectra of 1-chloro-5,6-dihydro-6-[(benzylamino)methyl]-5-(3-chloro phenyl)pyrrolo[1,2-f]pteridine 5.13a. 


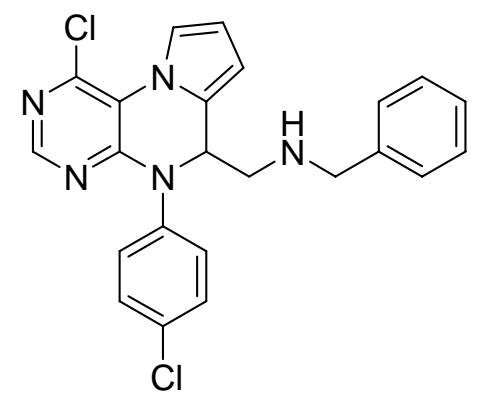

$\mathrm{C}_{23} \mathrm{H}_{19} \mathrm{Cl}_{2} \mathrm{~N}_{5}$

Exact Mass: 435.1

5.14a Mol. Wt.: 436.34
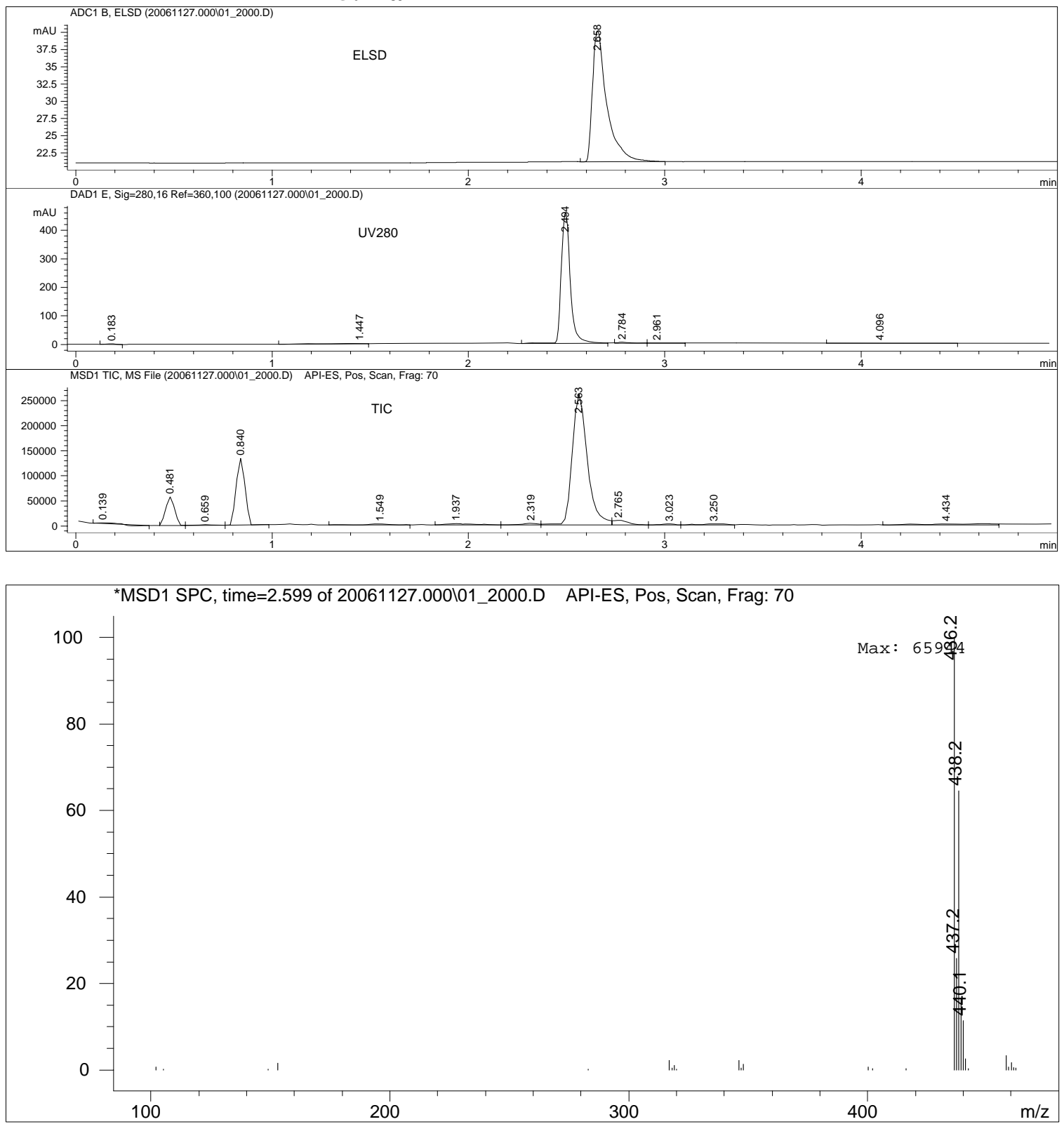

Fig. S-49: LC-MS-ELSD of 1-chloro-5,6-dihydro-6-[(benzylamino)methyl]-5-(4chlorophenyl)pyrrolo[1,2-f]pteridine 5.14a. 
$000 \cdot 0$

$6 s 2 \cdot 1$
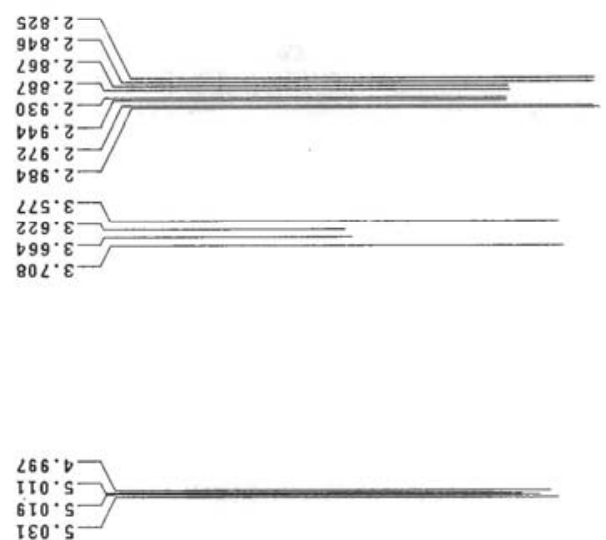

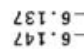

$62 b \cdot 9$
$680 \cdot 9$

$6 S 6 \cdot .9$
860.2

$860^{\circ}<$

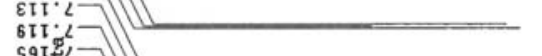

CLIOLL

$68 \mathrm{I}$ कर

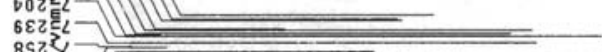

I925 -

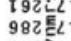

$\angle 0832$

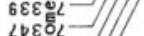

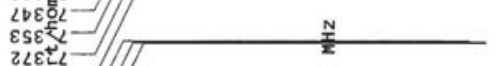

$\angle \angle \varepsilon$ व $=0$ =

$9866<-$

$\angle 86 \div$

021

के 15

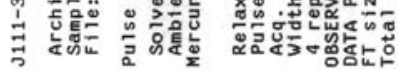
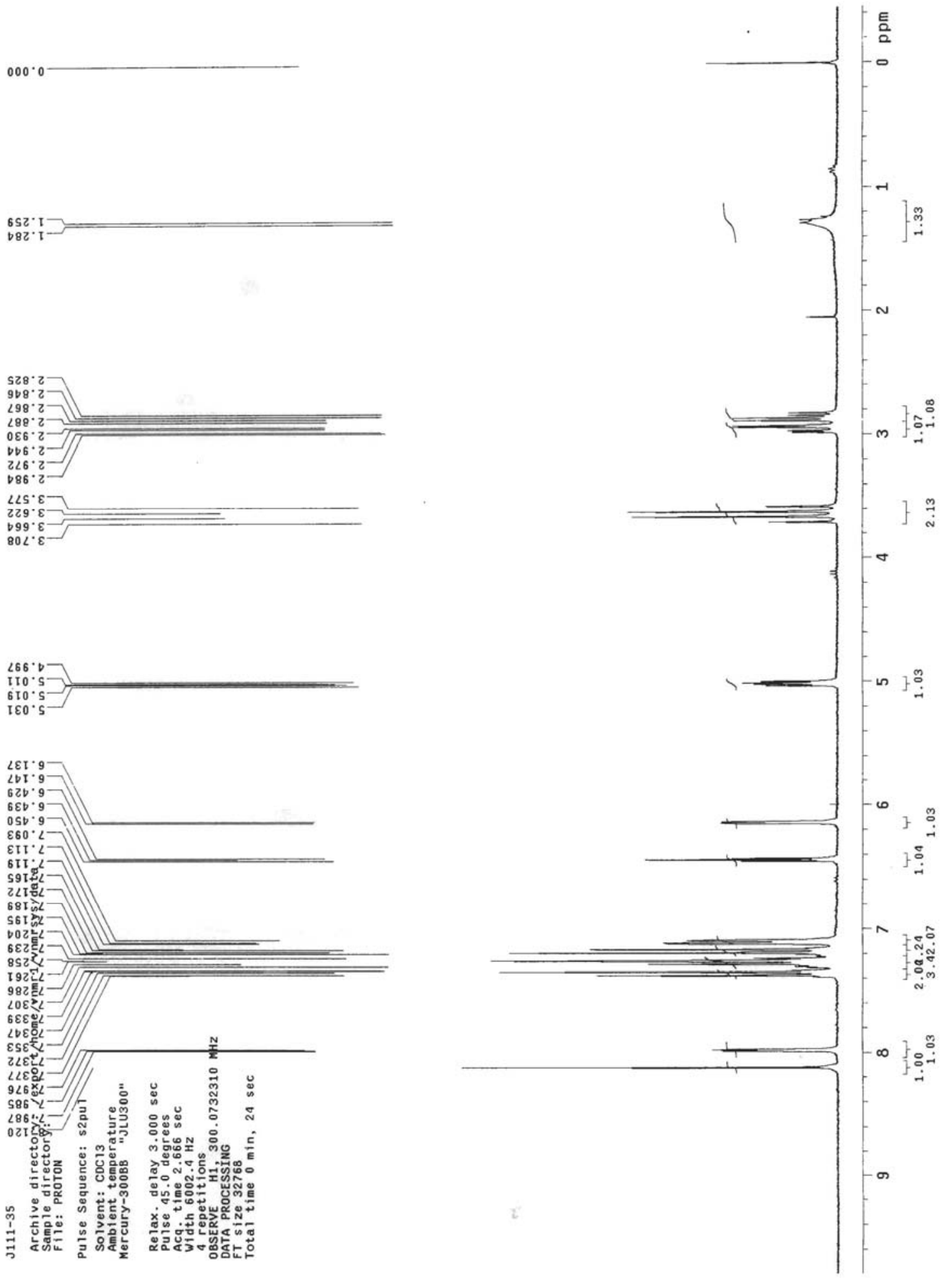

Fig. S-50: ${ }^{1} \mathrm{H}$ Spectra of 1-chloro-5,6-dihydro-6-[(benzylamino)methyl]-5-(4-chloro phenyl)pyrrolo[1,2-f]pteridine 5.14a. 


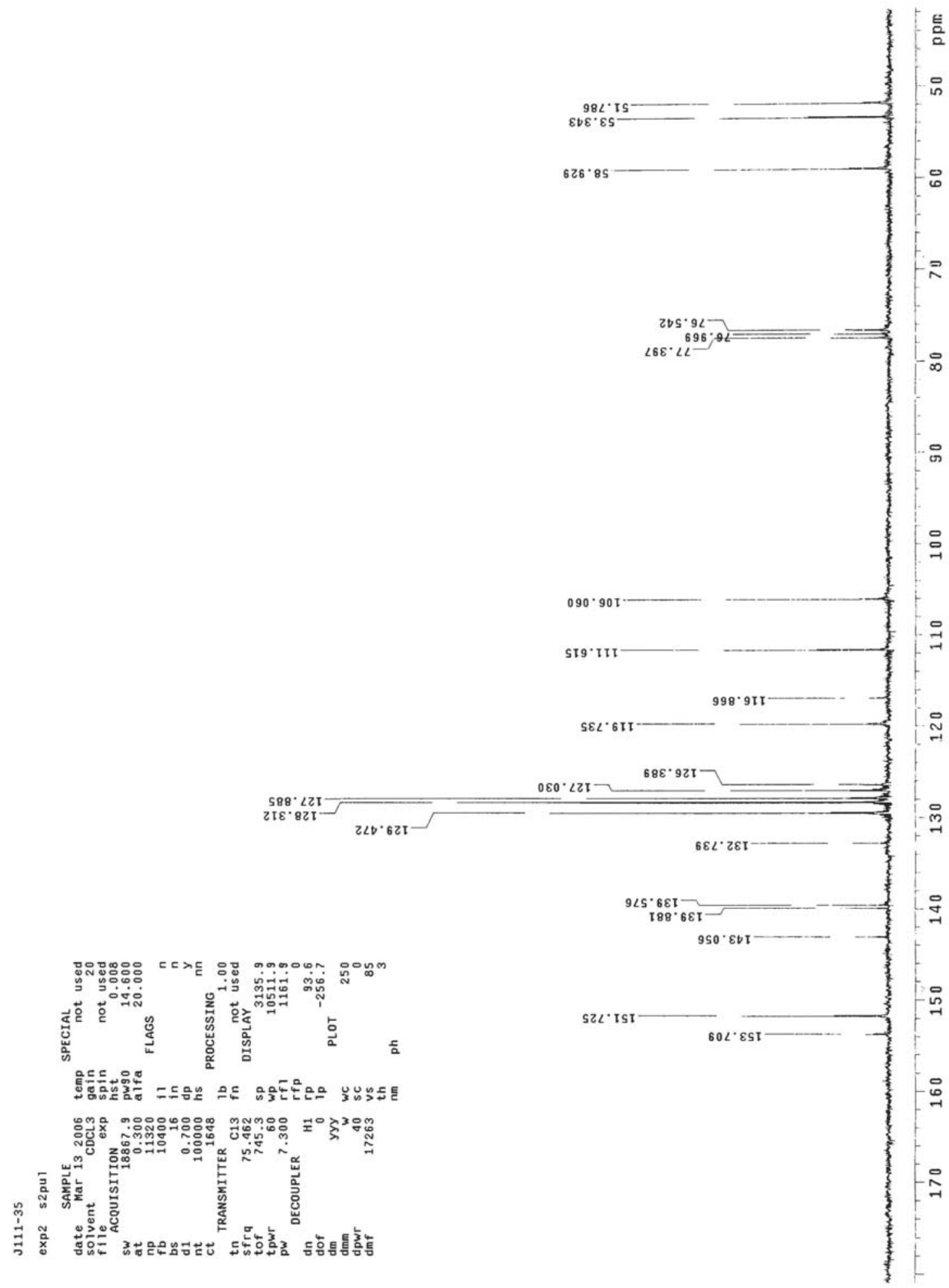

Fig. S-51: ${ }^{13} \mathrm{C}$ Spectra of 1-chloro-5,6-dihydro-6-[(benzylamino)methyl]-5-(4-chloro phenyl)pyrrolo[1,2-f]pteridine 5.14a. 


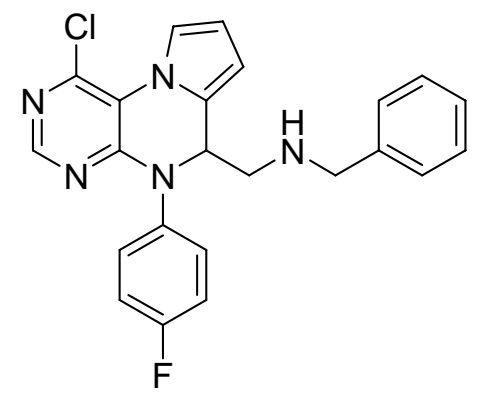

$\mathrm{C}_{23} \mathrm{H}_{19} \mathrm{ClFN}$

Exact Mass: 419.13

5.15a Mol. Wt.: 419.88
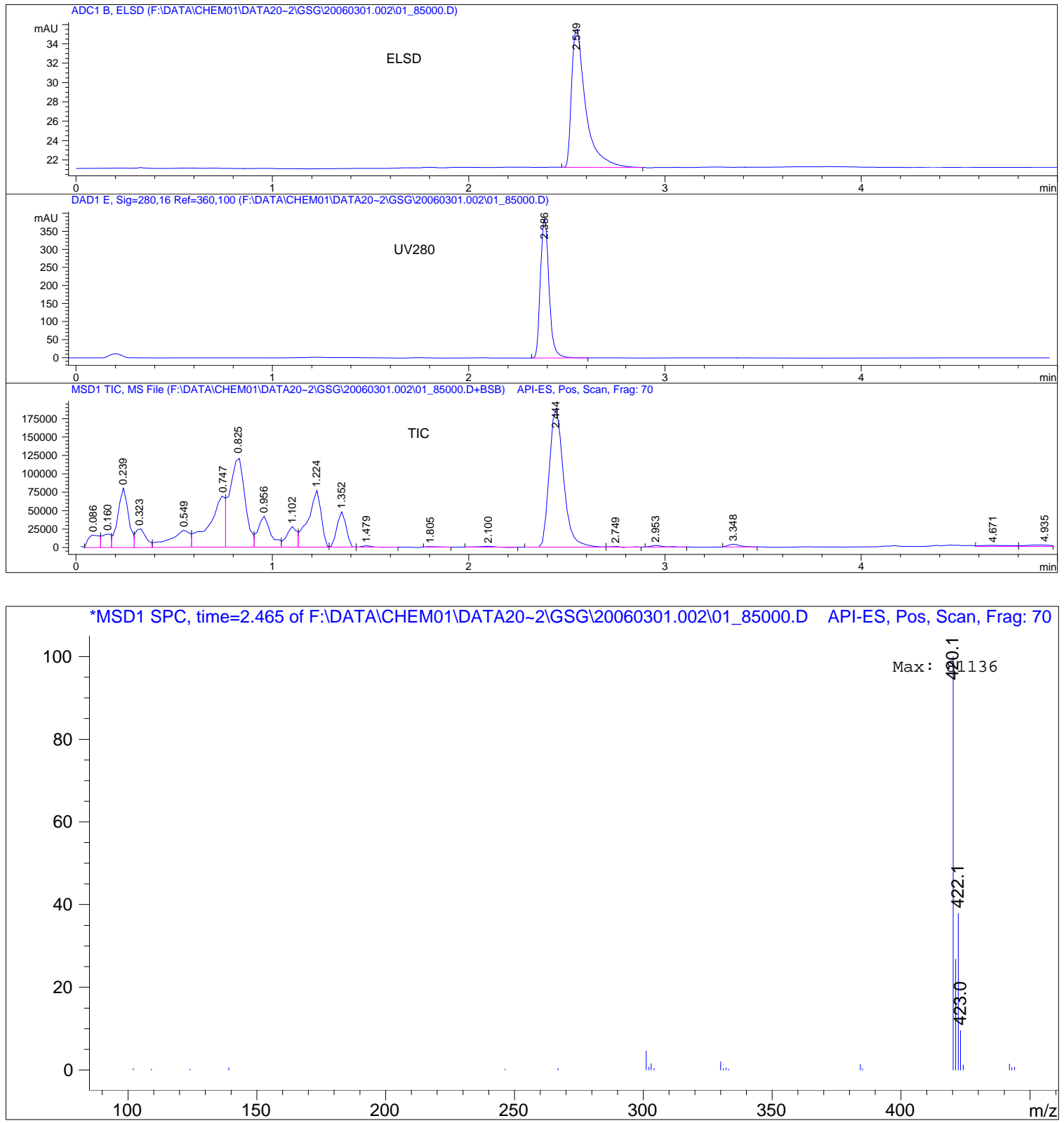

Fig. S-52: LC-MS-ELSD of 1-chloro-5,6-dihydro-6-[(benzylamino)methyl]-5-(4-floro phenyl)pyrrolo[1,2-f]pteridine 5.15a. 


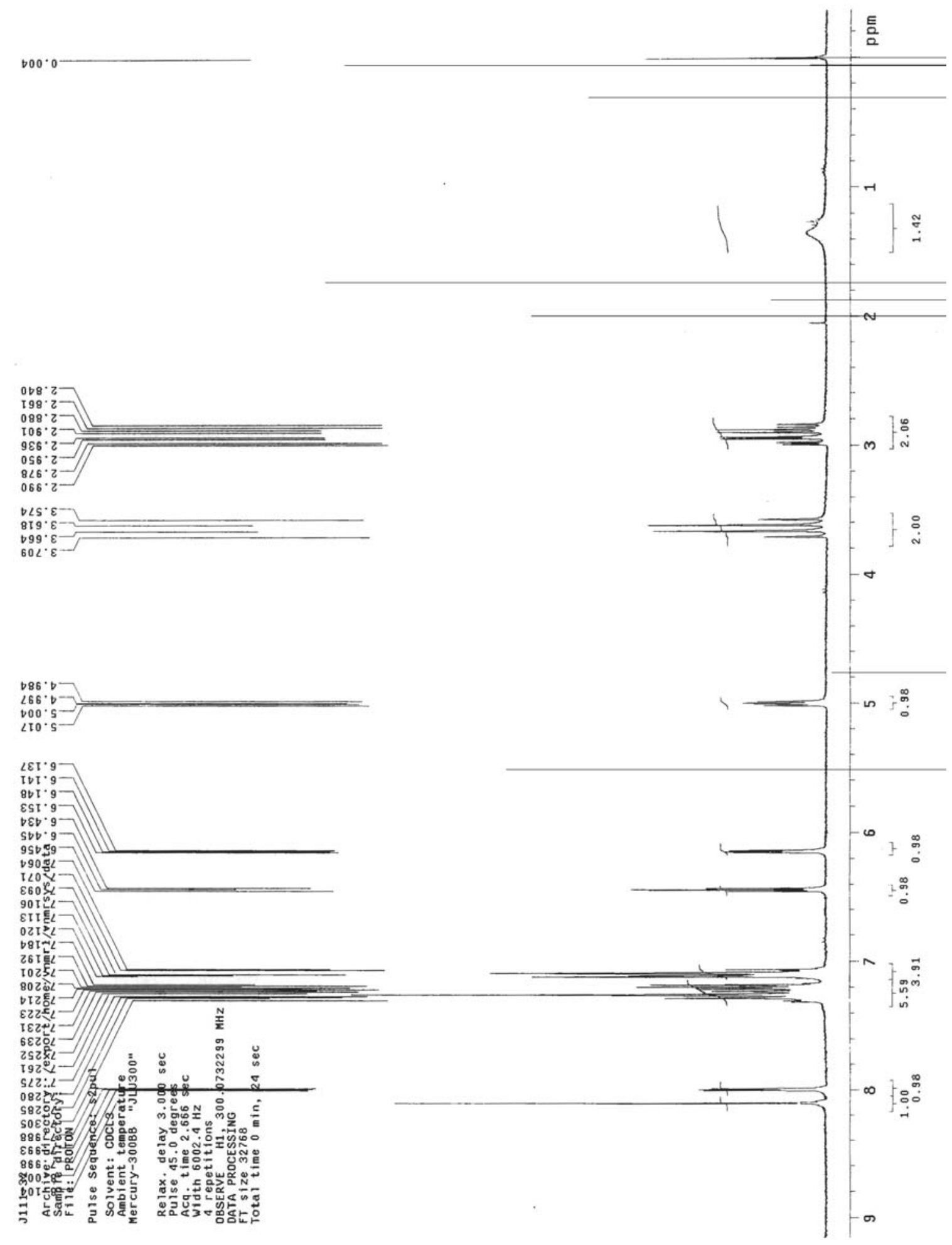

Fig. S-53: ${ }^{1} \mathrm{H}$ Spectra of 1-chloro-5,6-dihydro-6-[(benzylamino)methyl]-5-(4-floro phenyl)pyrrolo[1,2-f]pteridine 5.15a. 


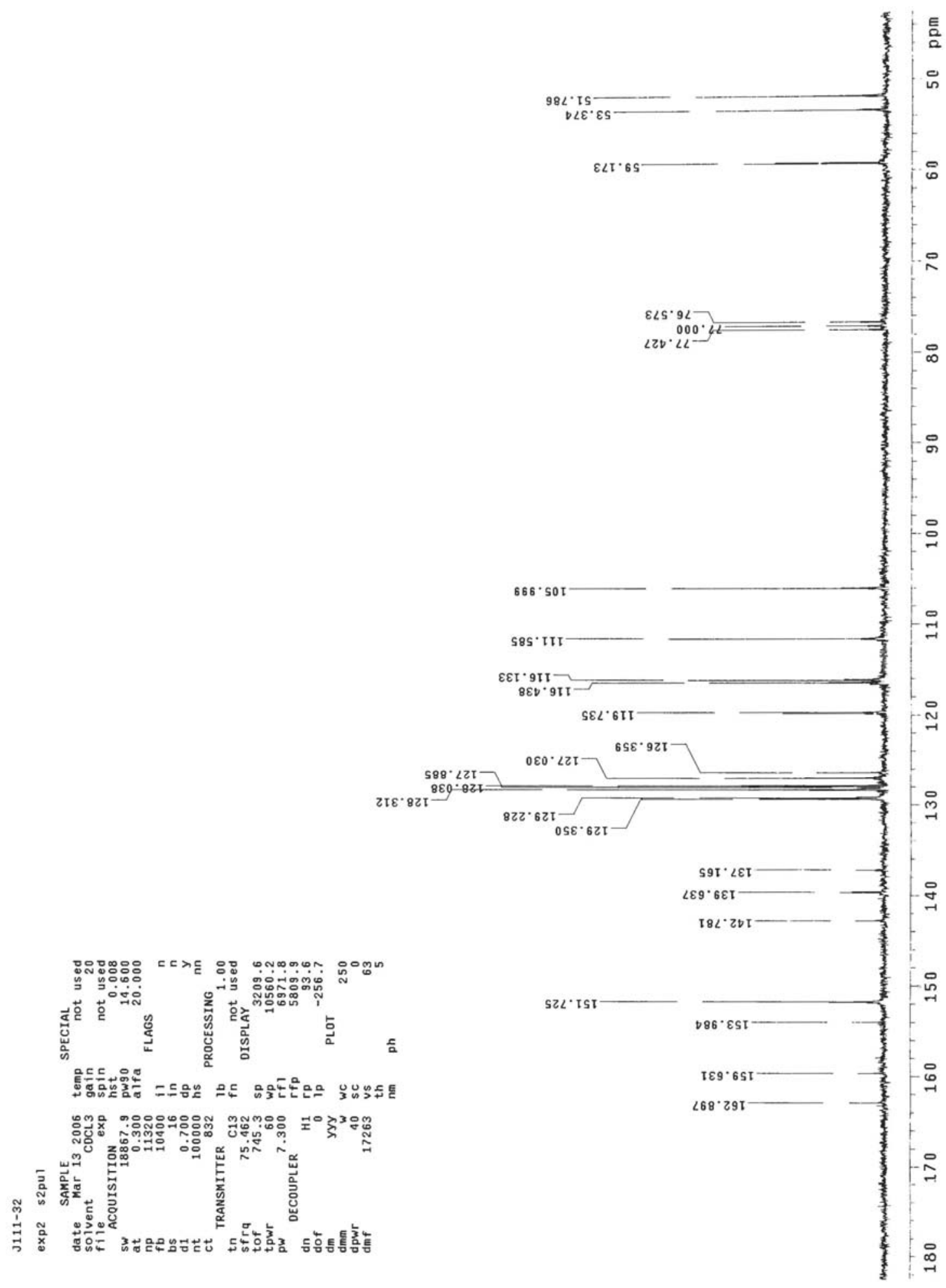

Fig. S-54: ${ }^{13} \mathrm{C}$ Spectra of 1-chloro-5,6-dihydro-6-[(benzylamino)methyl]-5-(4-floro phenyl)pyrrolo[1,2-f]pteridine 5.15a. 


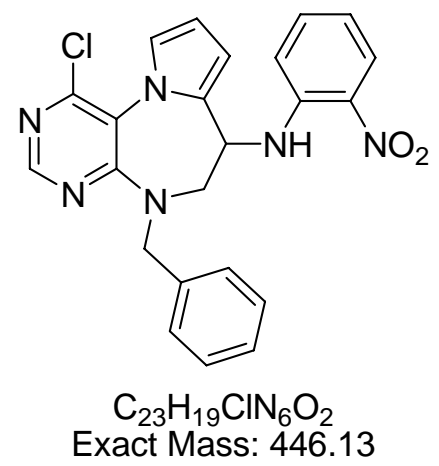

4.16a
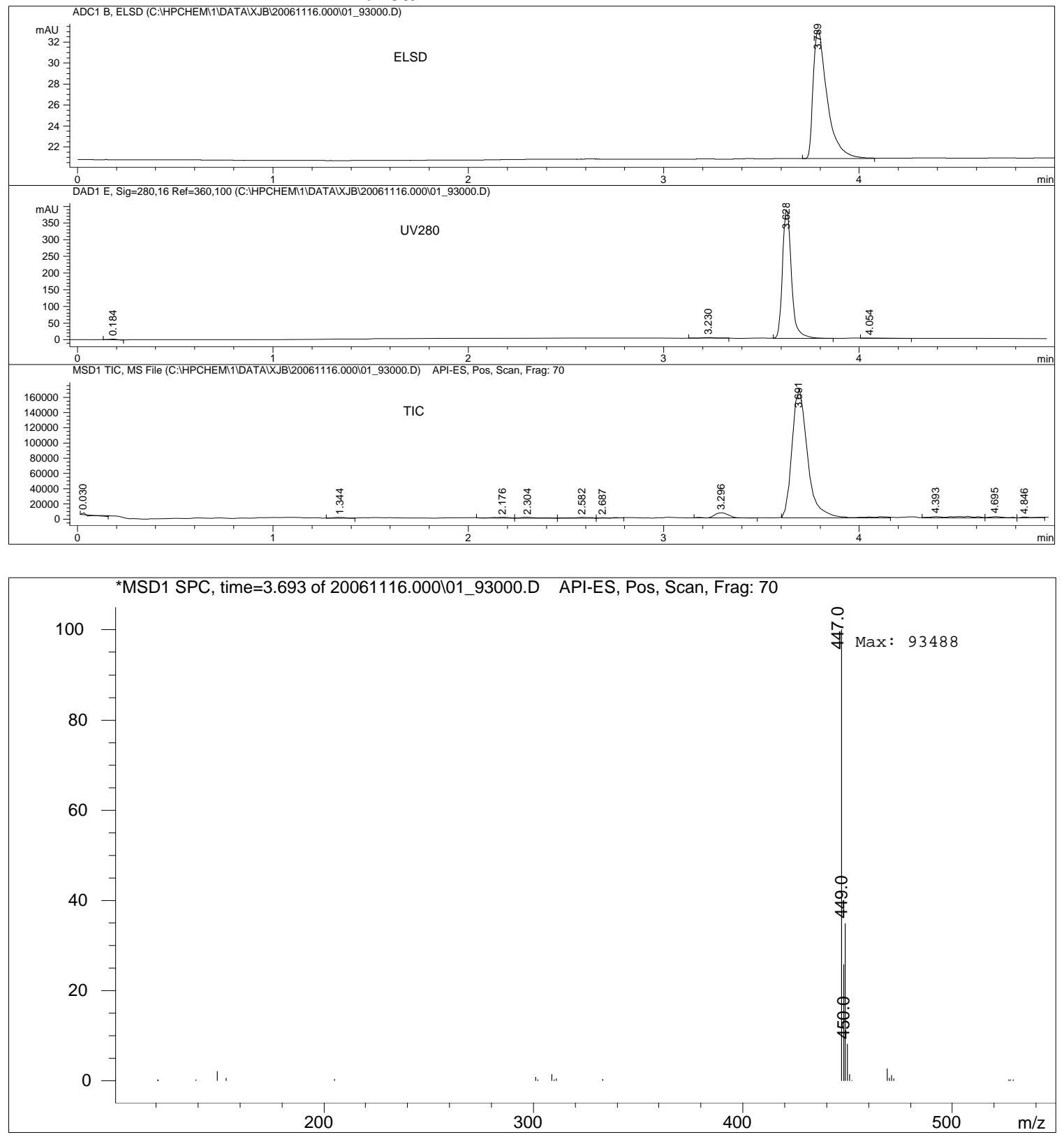

Fig. S-55: LC-MS-ELSD of 7-(2-nitrophenylamino)-5-benzyl-1-chloro-6,7-dihydro pyrrolo[2,1-d][1,5]pyrimidodiazepine 4.16a. 


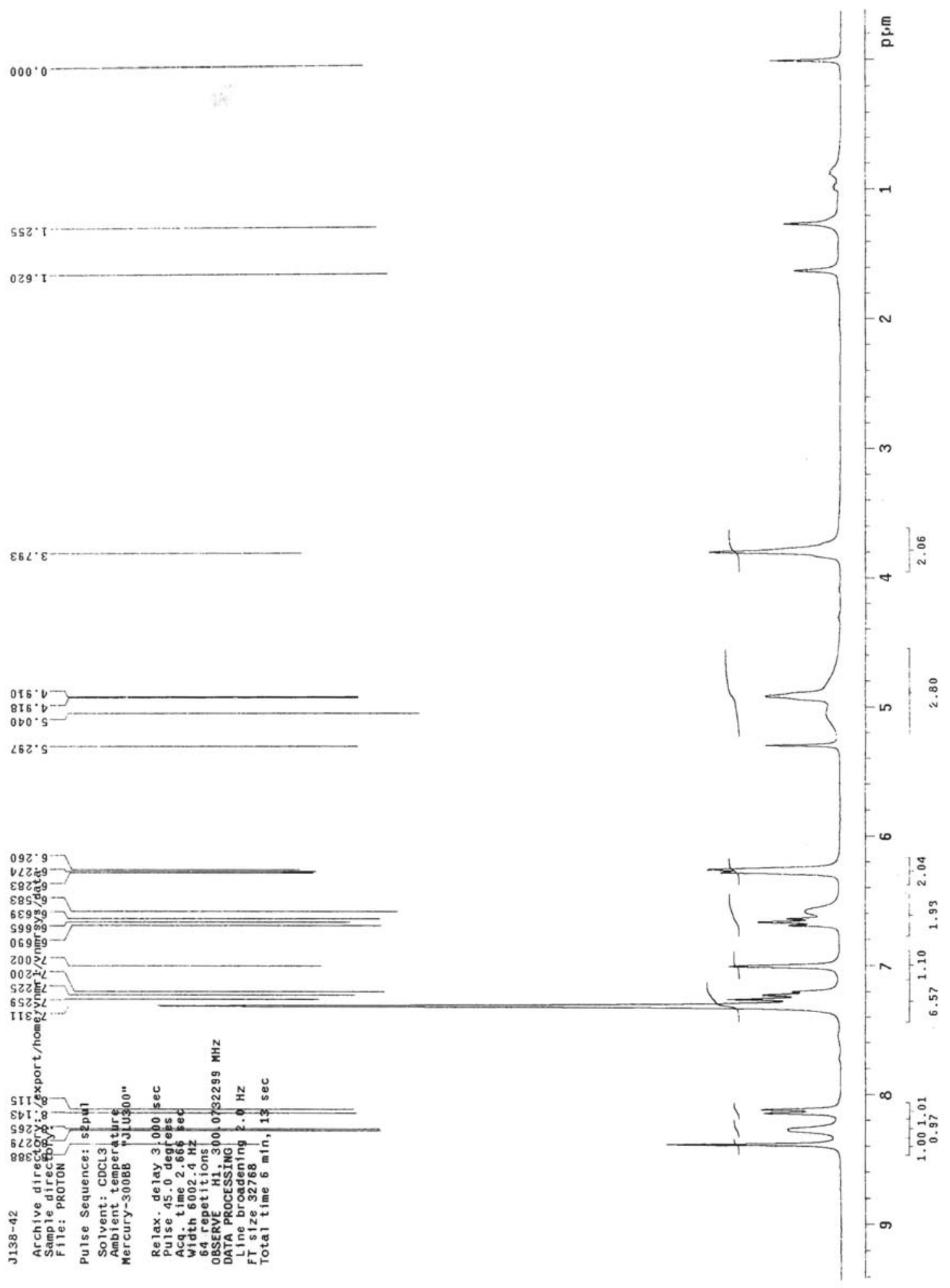

Fig. S-56: ${ }^{1} \mathrm{H}$ Spectra of 7-(2-nitrophenylamino)-5-benzyl-1-chloro-6,7-dihydro pyrrolo[2,1-d][1,5]pyrimidodiazepine 4.16a. 


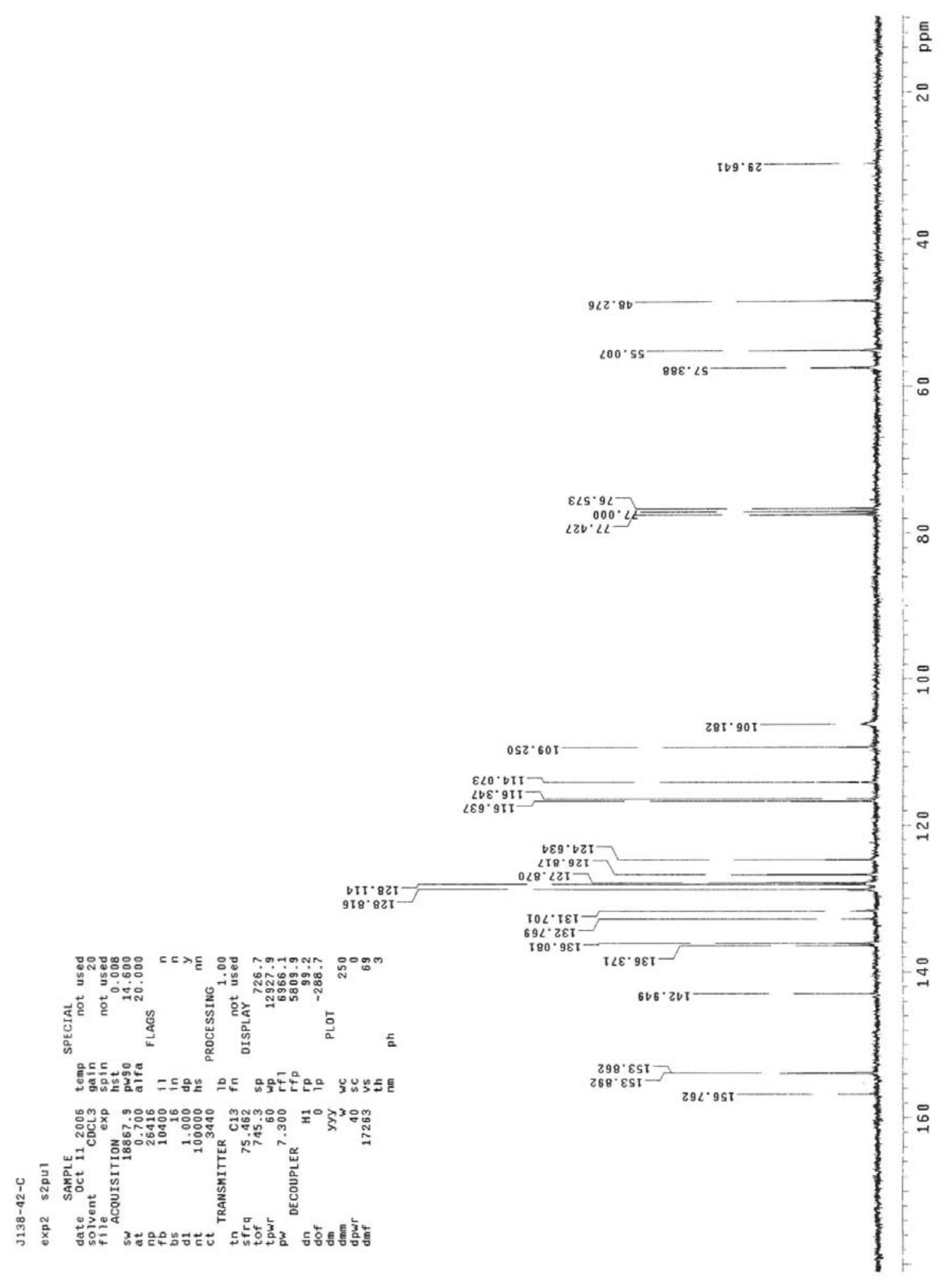

Fig. S-57: ${ }^{13} \mathrm{C}$ Spectra of 7-(2-nitrophenylamino)-5-benzyl-1-chloro-6,7-dihydro pyrrolo[2,1-d][1,5]pyrimidodiazepine 4.16a. 


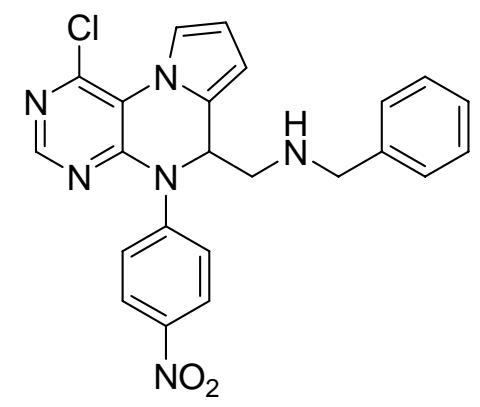

$\mathrm{C}_{23} \mathrm{H}_{19} \mathrm{ClN}_{6} \mathrm{O}_{2}$

Exact Mass: 446.13

$5.17 a$

Mol. Wt.: 446.89
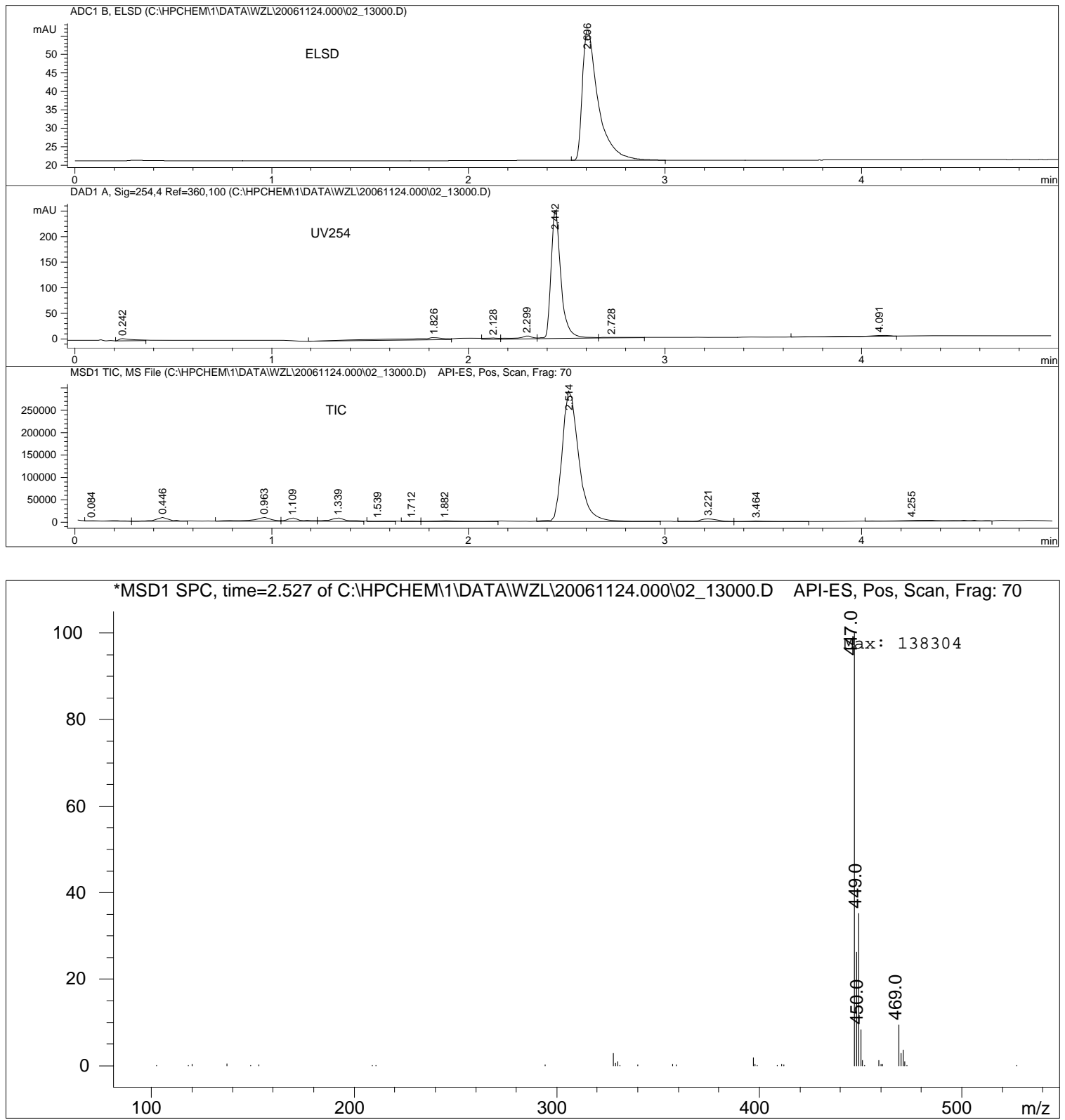

Fig. S-58: LC-MS-ELSD of 1-chloro-5,6-dihydro-6-[(benzylamino)methyl]-5-(4nitrophenyl)pyrrolo[1,2-f]pteridine 5.17a. 


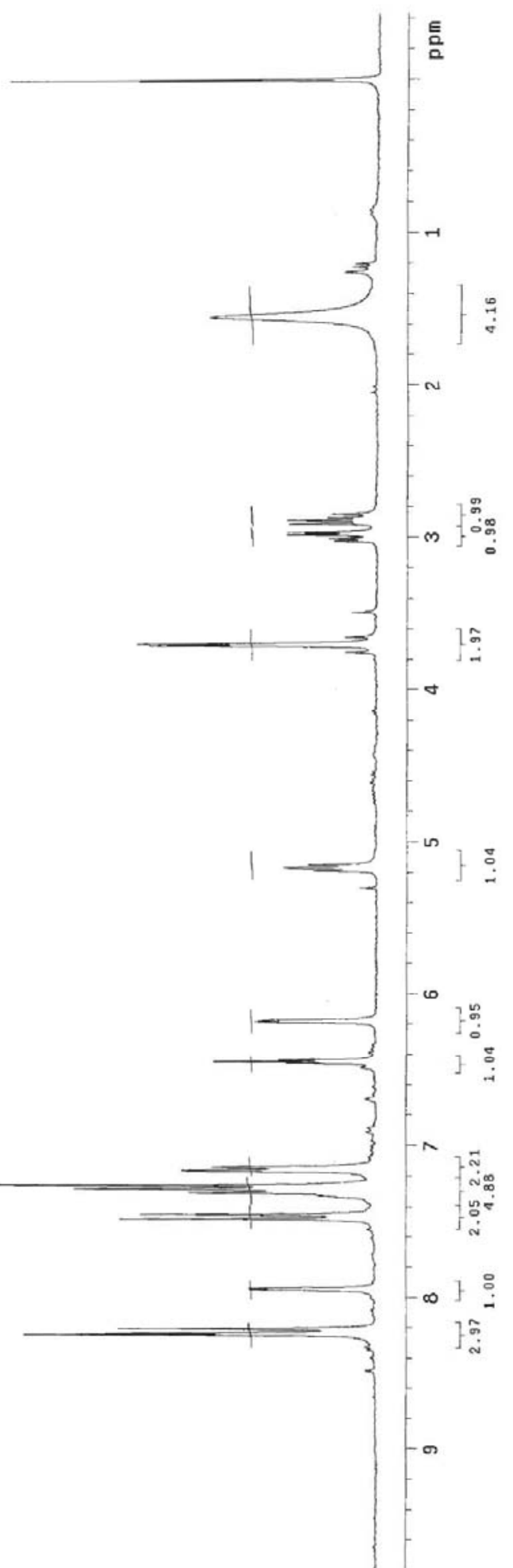

$6 \mathrm{DT} \cdot \mathrm{s}$
$99 \mathrm{~T} \cdot \mathrm{s}$ $99 \mathrm{I} \cdot \mathrm{s}$
$88 \mathrm{I} \cdot \mathrm{s}$
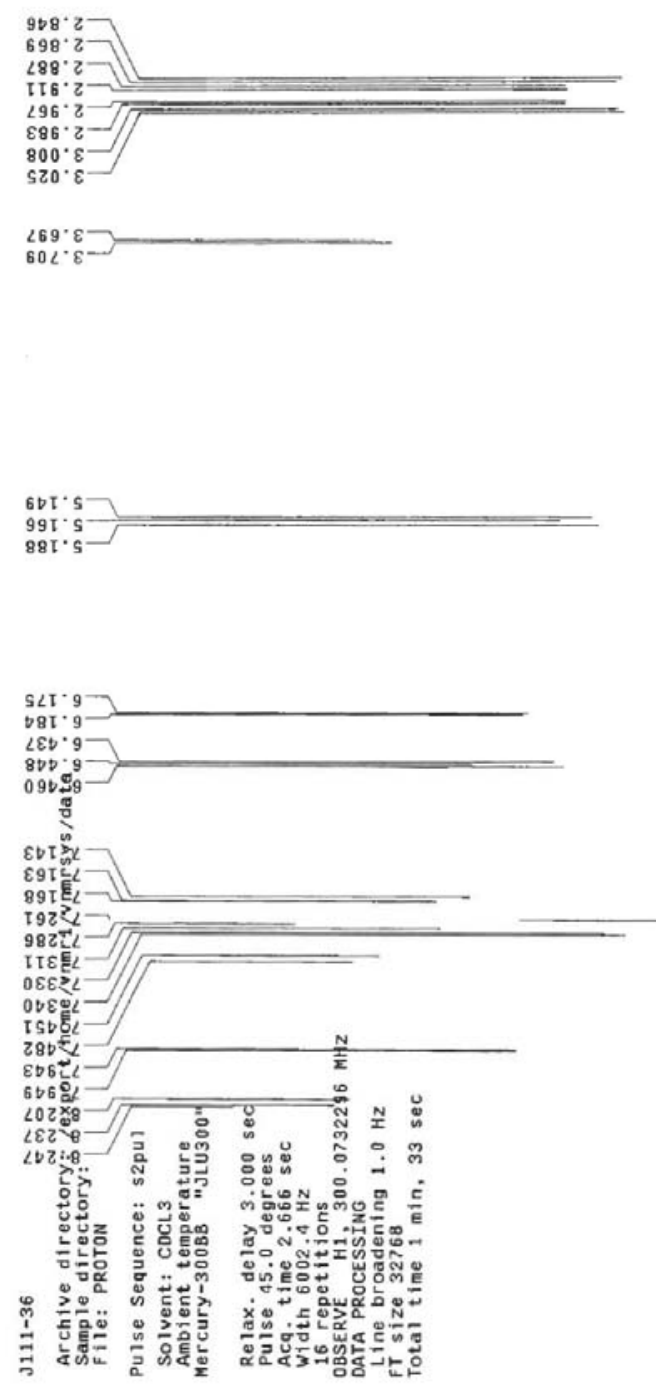

Fig. S-59: ${ }^{1} \mathrm{H}$ Spectra of 1-chloro-5,6-dihydro-6-[(benzylamino)methyl]-5-(4-nitro phenyl)pyrrolo[1,2-f]pteridine 5.17a. 


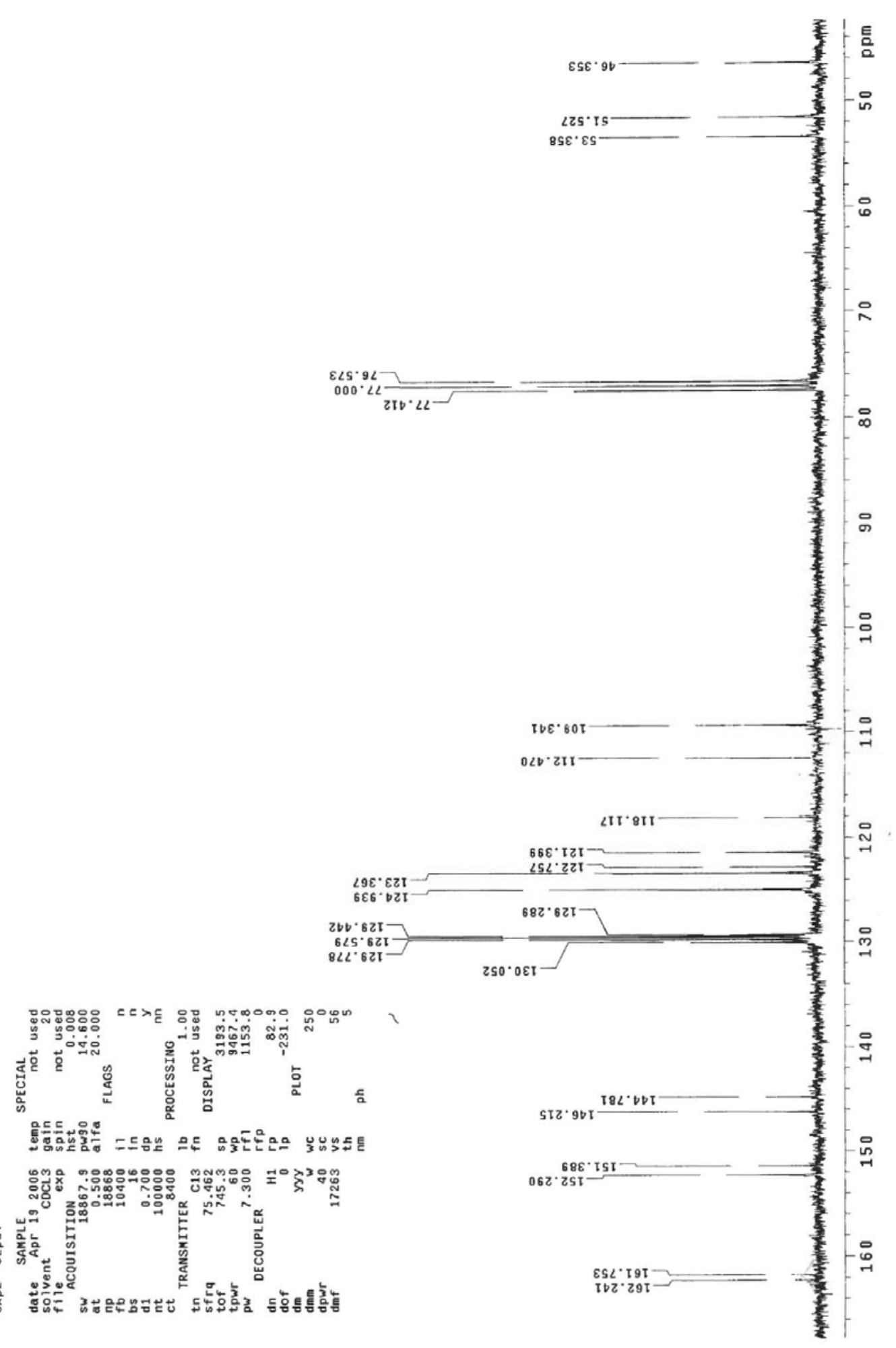

Fig. S-60: ${ }^{13} \mathrm{C}$ Spectra of 1-chloro-5,6-dihydro-6-[(benzylamino)methyl]-5-(4-nitro phenyl)pyrrolo[1,2-f]pteridine 5.17a. 


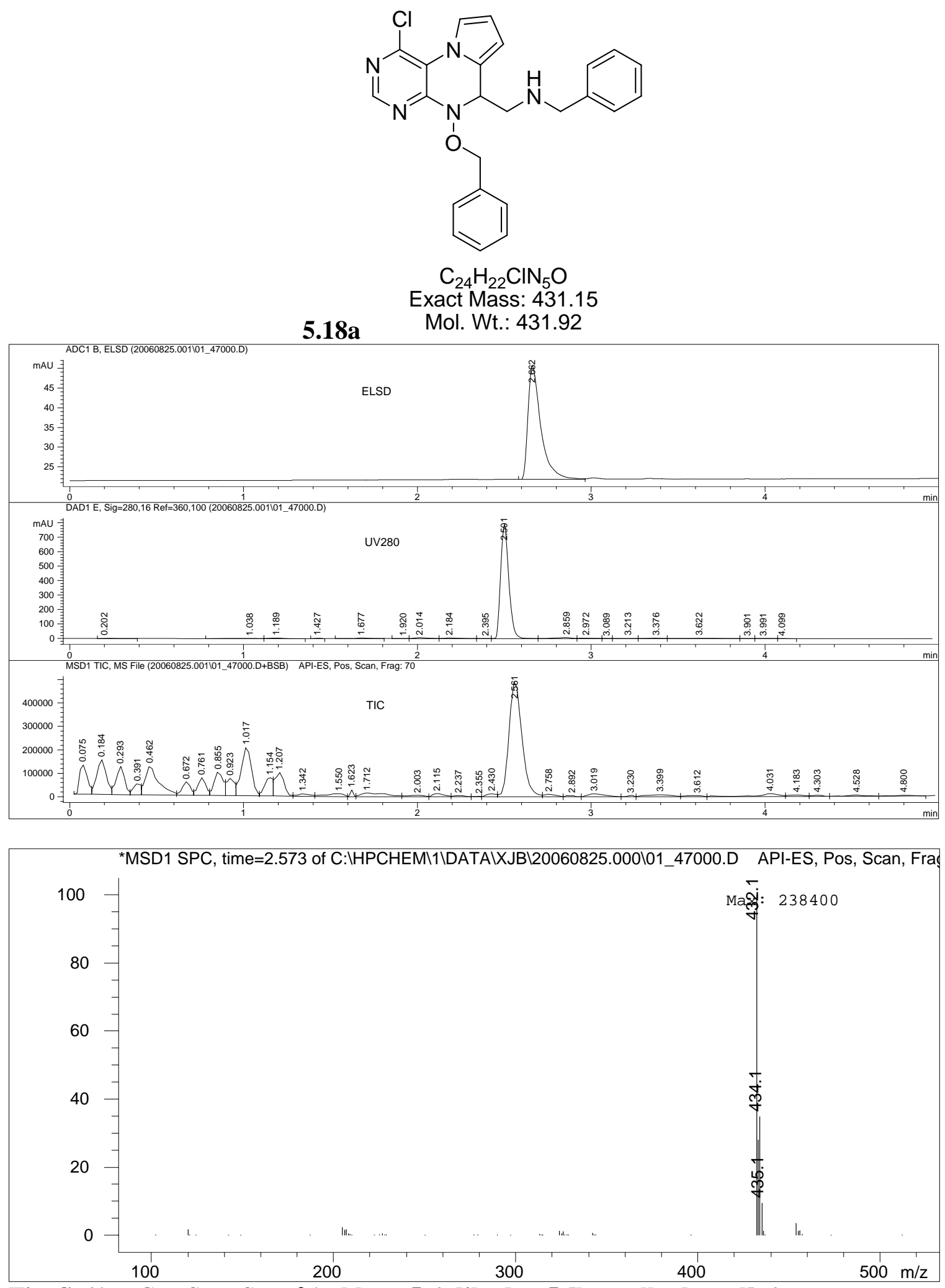

Fig. S-61: LC-MS-ELSD of 1-chloro-5,6-dihydro-5-[benzylhydroxyl]-6[(benzylamino)methyl]pyrrolo[1,2-f]pteridine 5.18a. 
$000 \cdot 0$

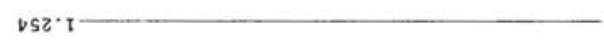

$208^{\circ} \mathrm{I}$
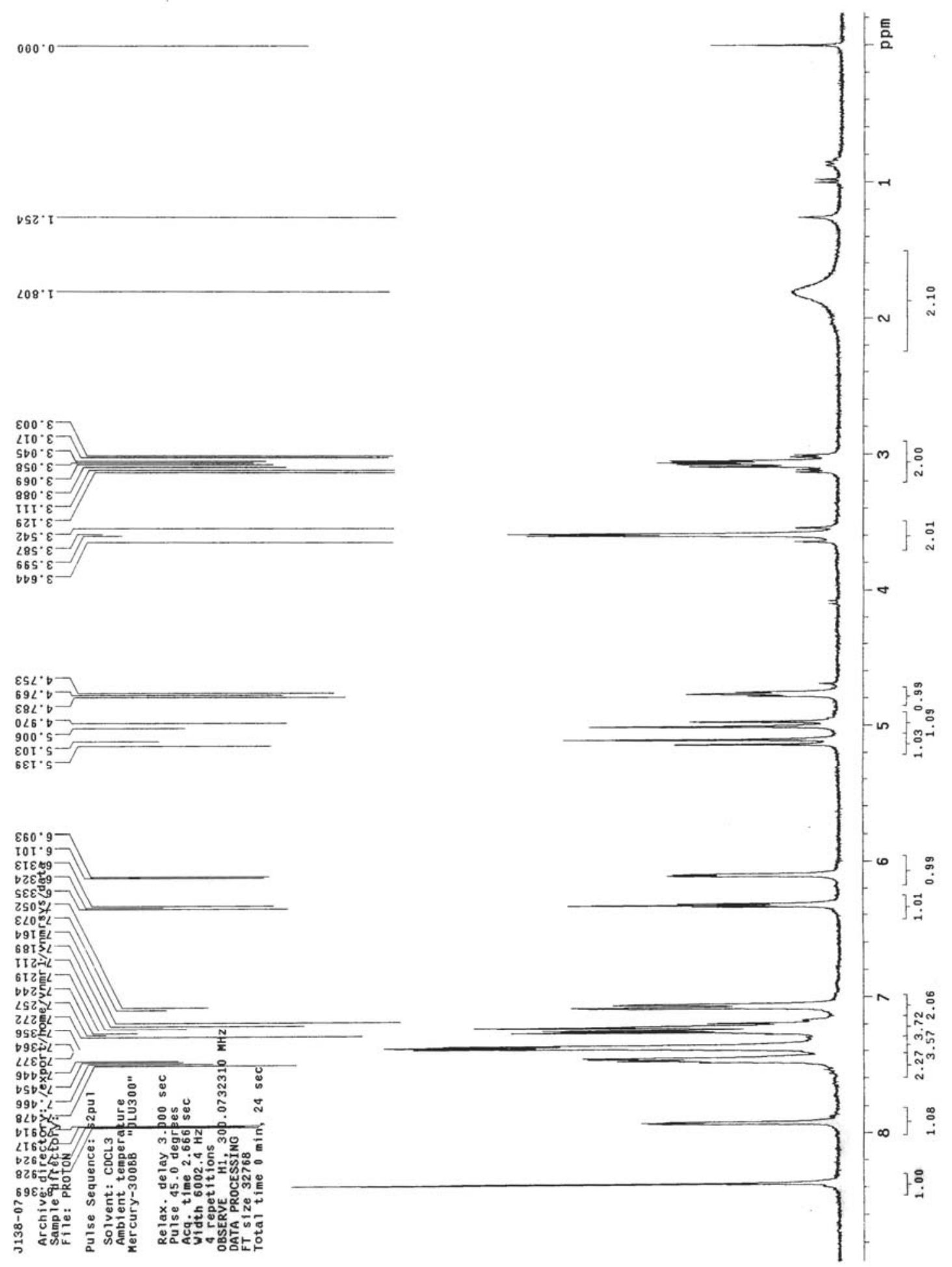

Fig. S-62: ${ }^{1} \mathrm{H}$ Spectra of 1-chloro-5,6-dihydro-5-[benzylhydroxyl]-6-[(benzylamino) methyl]pyrrolo[1,2-f]pteridine 5.18a. 


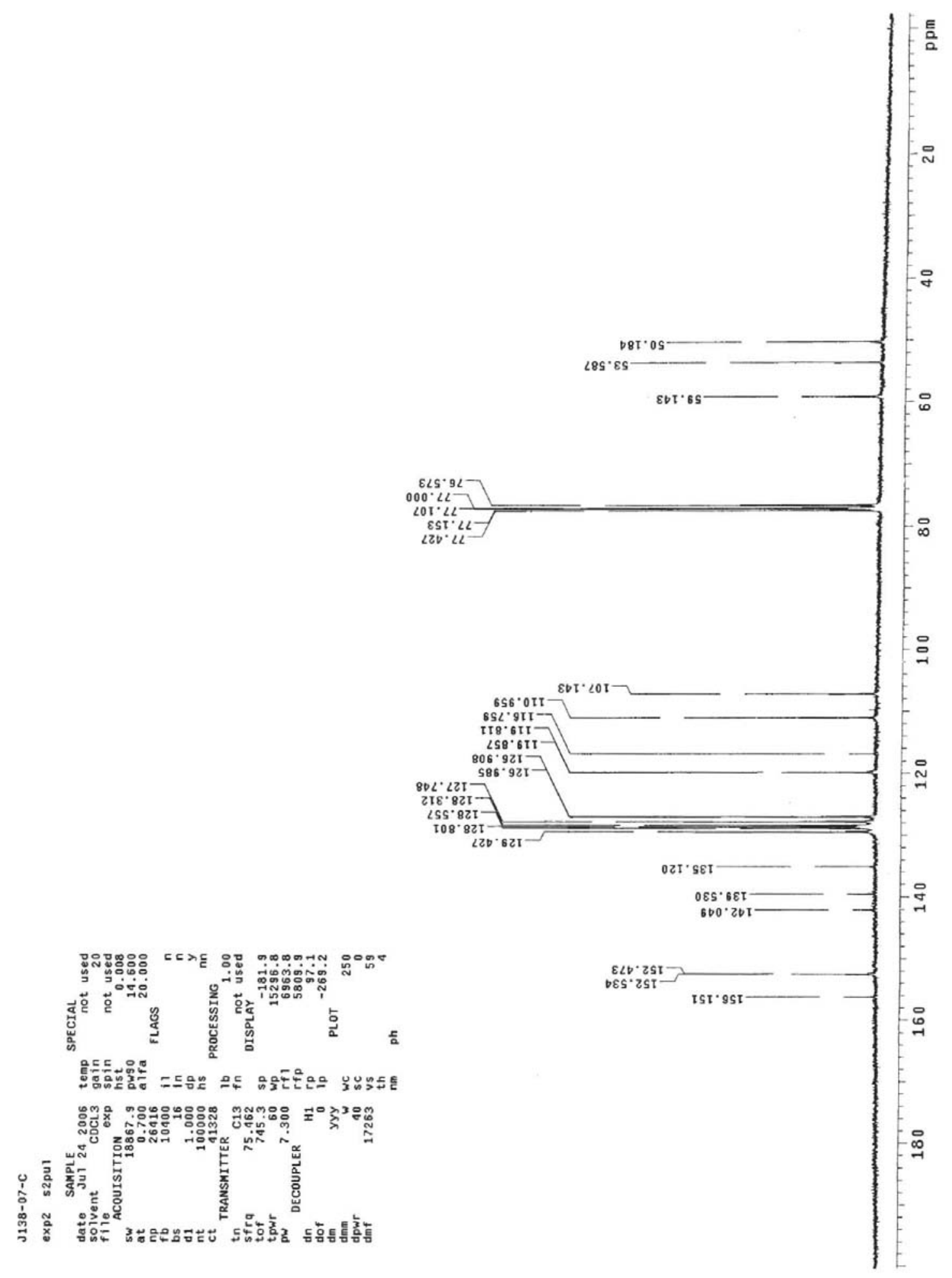

Fig. S-63: ${ }^{13} \mathrm{C}$ Spectra of 1-chloro-5,6-dihydro-5-[benzylhydroxyl]-6-[(benzylamino) methyl]pyrrolo[1,2-f]pteridine 5.18a. 


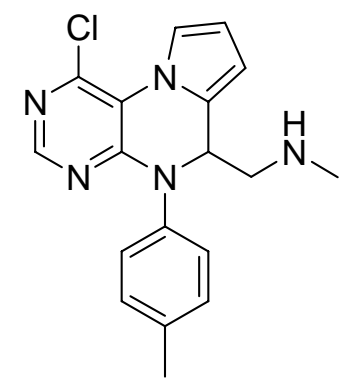

$\mathrm{C}_{18} \mathrm{H}_{18} \mathrm{ClN}_{5}$

Exact Mass: 339.13

5.10b Mol. Wt.: 339.82
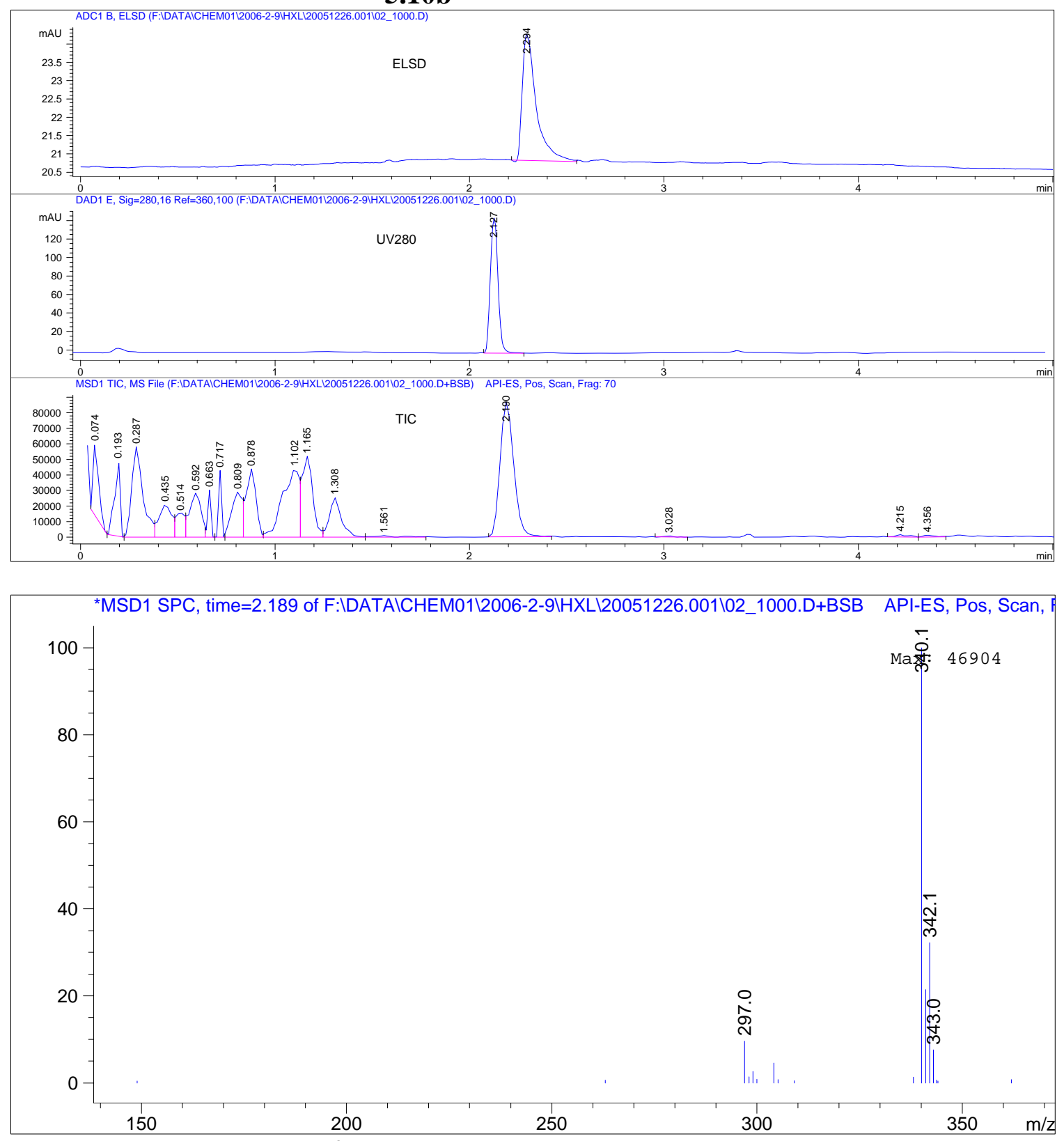

Fig. S-64: LC-MS-ELSD of 1-chloro-5,6-dihydro-6-[(methylamino)methyl]-5-(4methylphenyl)pyrrolo[1,2-f]pteridine $5.10 \mathrm{~b}$. 


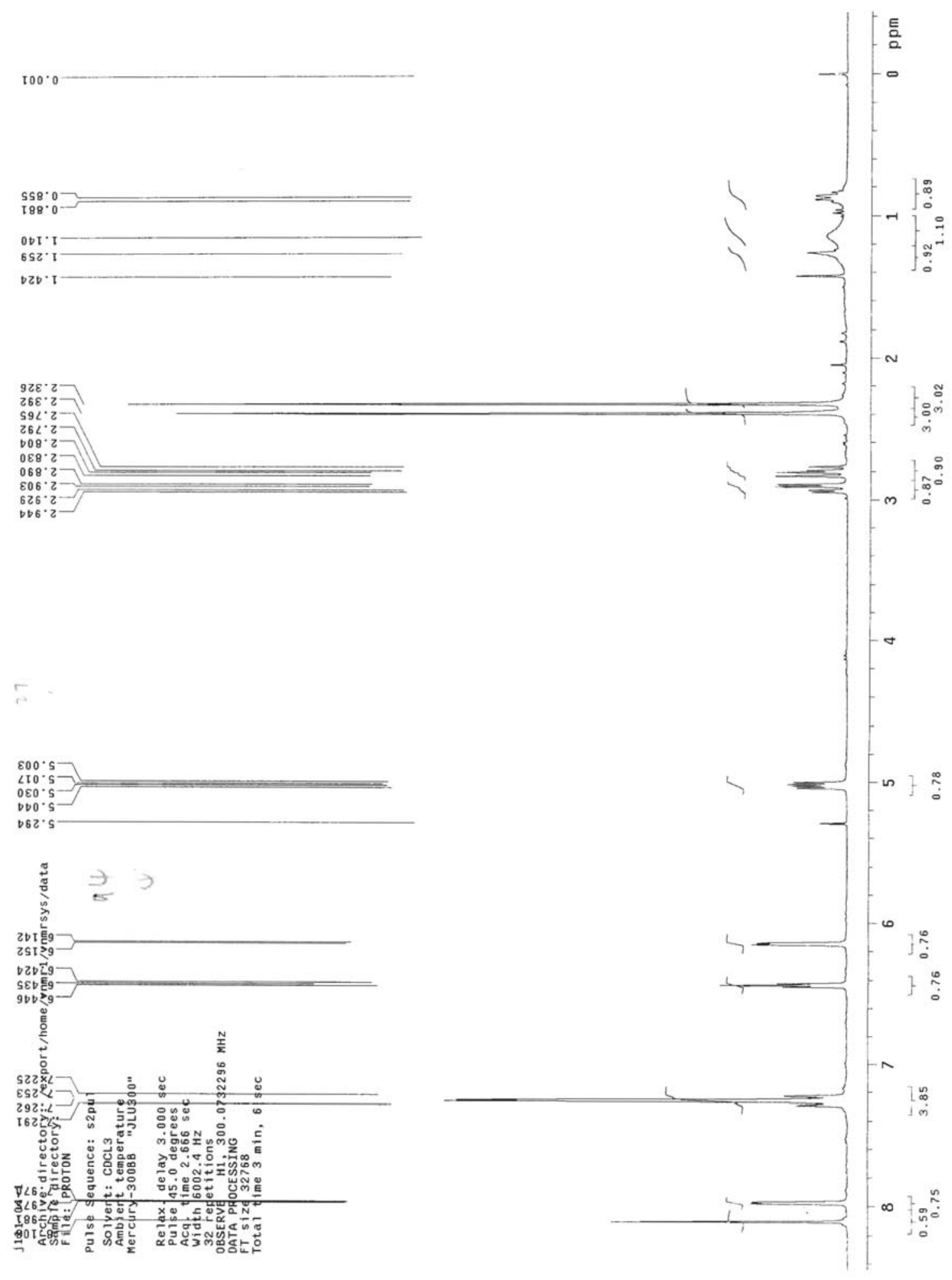

Fig. S-65: ${ }^{1} \mathrm{H}$ Spectra of 1-chloro-5,6-dihydro-6-[(methylamino)methyl]-5-(4-methyl phenyl)pyrrolo[1,2-f]pteridine 5.10b. 


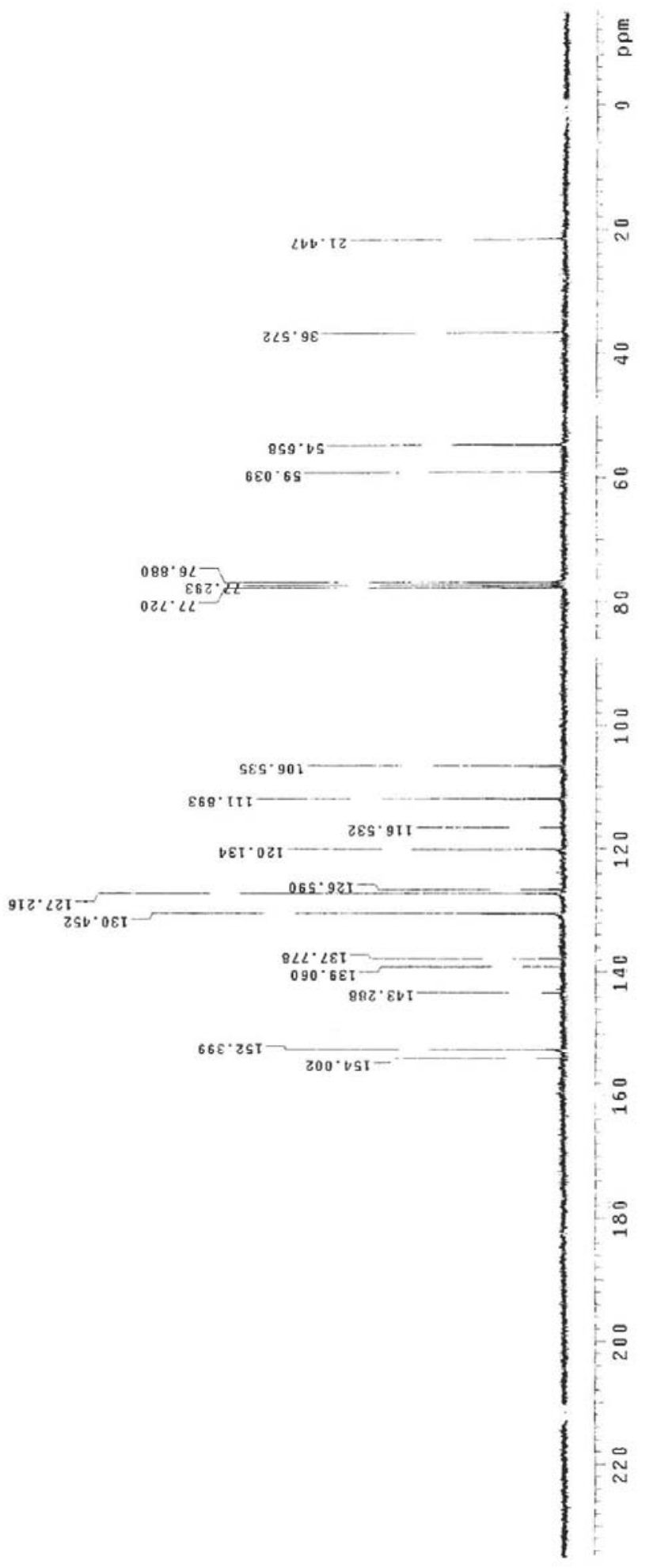

Fig. S-66: ${ }^{13} \mathrm{C}$ Spectra of 1-chloro-5,6-dihydro-6-[(methylamino)methyl]-5-(4-methyl phenyl)pyrrolo[1,2-f]pteridine $5.10 \mathrm{~b}$. 
<smiles>CNCC1c2cccn2-c2c(Cl)ncnc2N1c1ccc(Cl)cc1</smiles>

$\mathrm{C}_{17} \mathrm{H}_{15} \mathrm{Cl}_{2} \mathrm{~N}_{5}$

Exact Mass: 359.07

5.14b Mol. Wt.: 360.24
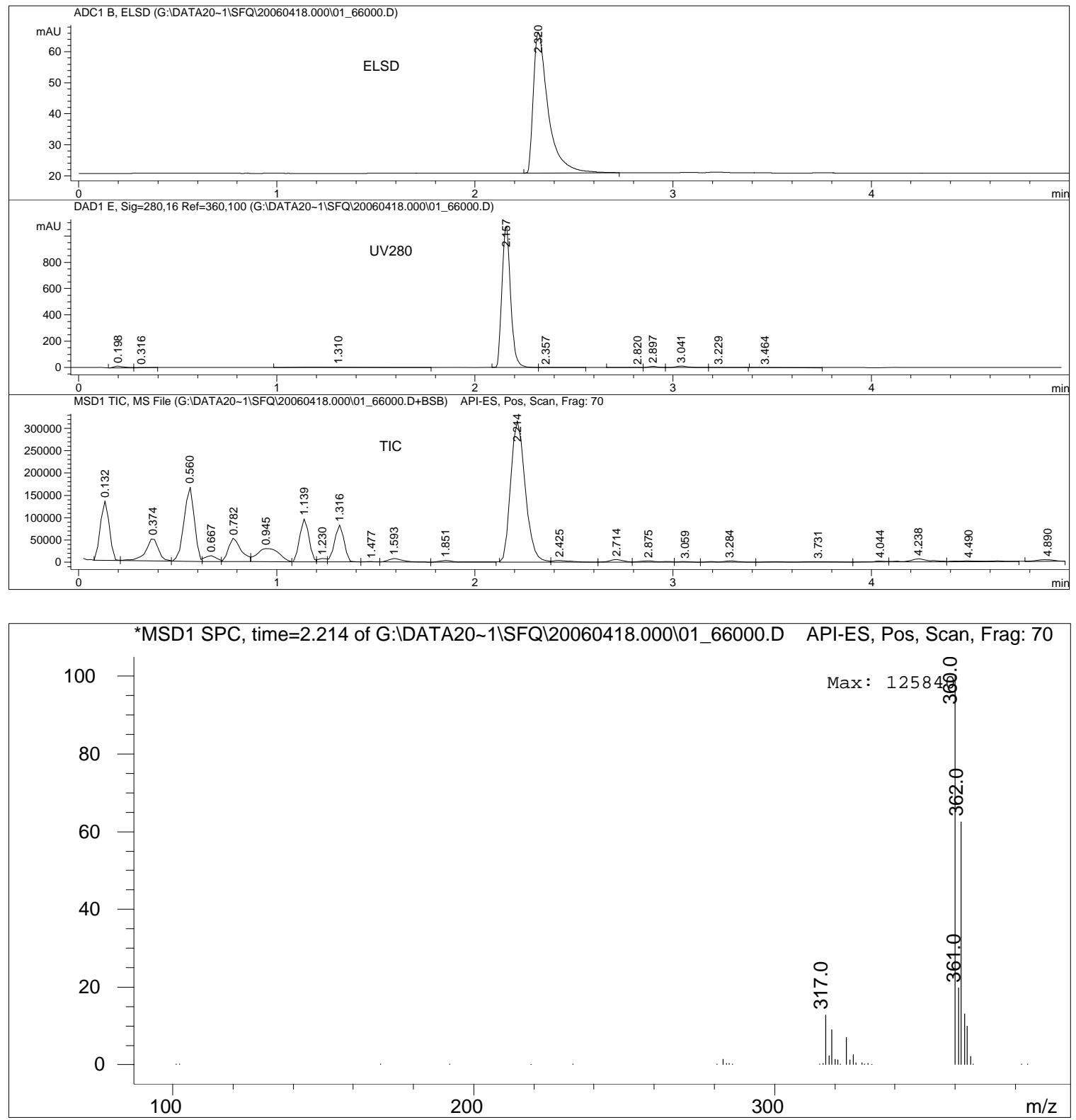

Fig. S-67: LC-MS-ELSD of 1-chloro-5,6-dihydro-6-[(methylamino)methyl]-5-(4chlorophenyl)pyrrolo[1,2-f]pteridine $5.14 \mathrm{~b}$. 


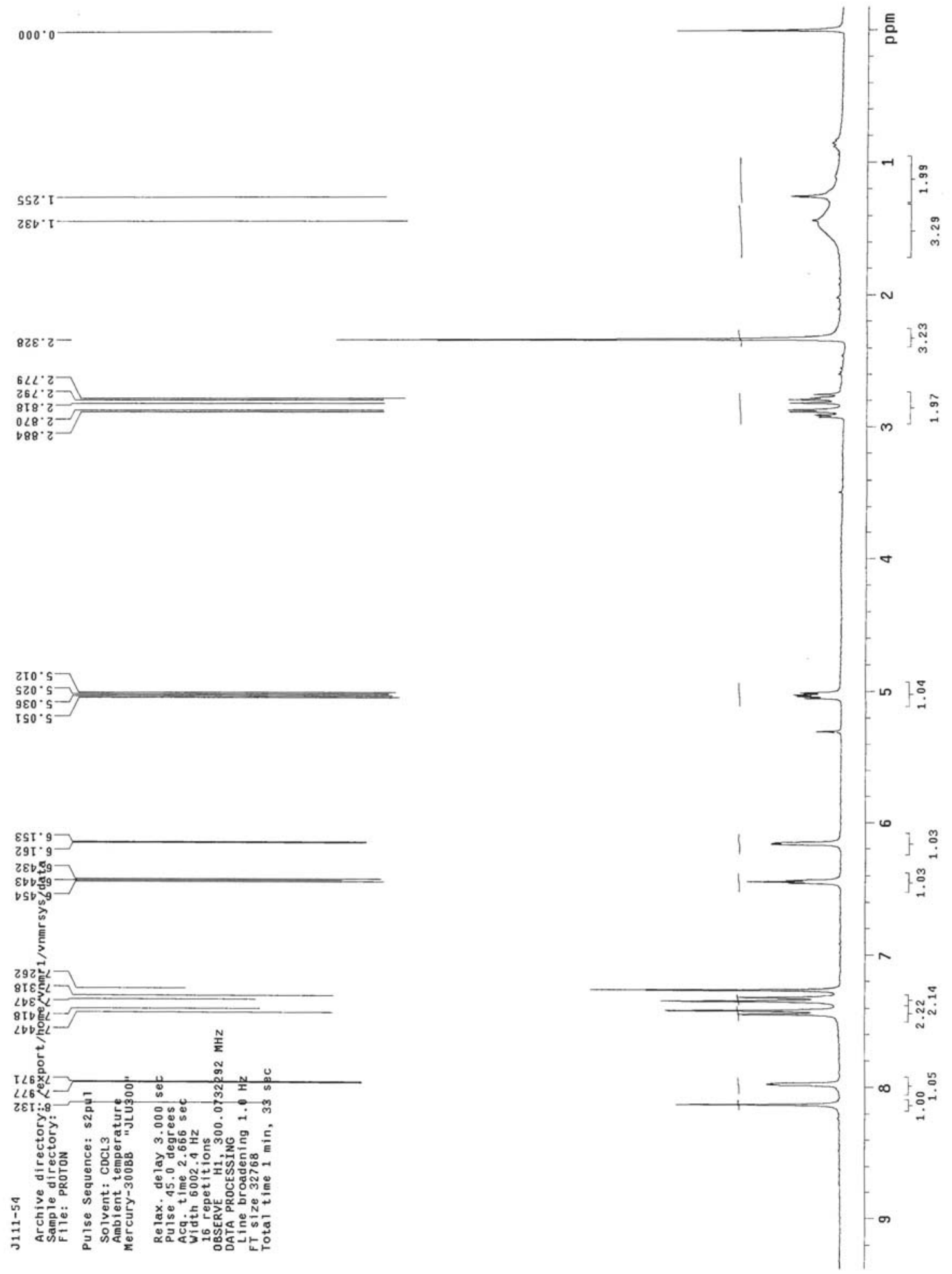

Fig. S-68: ${ }^{1} \mathrm{H}$ Spectra of 1-chloro-5,6-dihydro-6-[(methylamino)methyl]-5-(4-chloro phenyl)pyrrolo[1,2-f]pteridine 5.14b. 

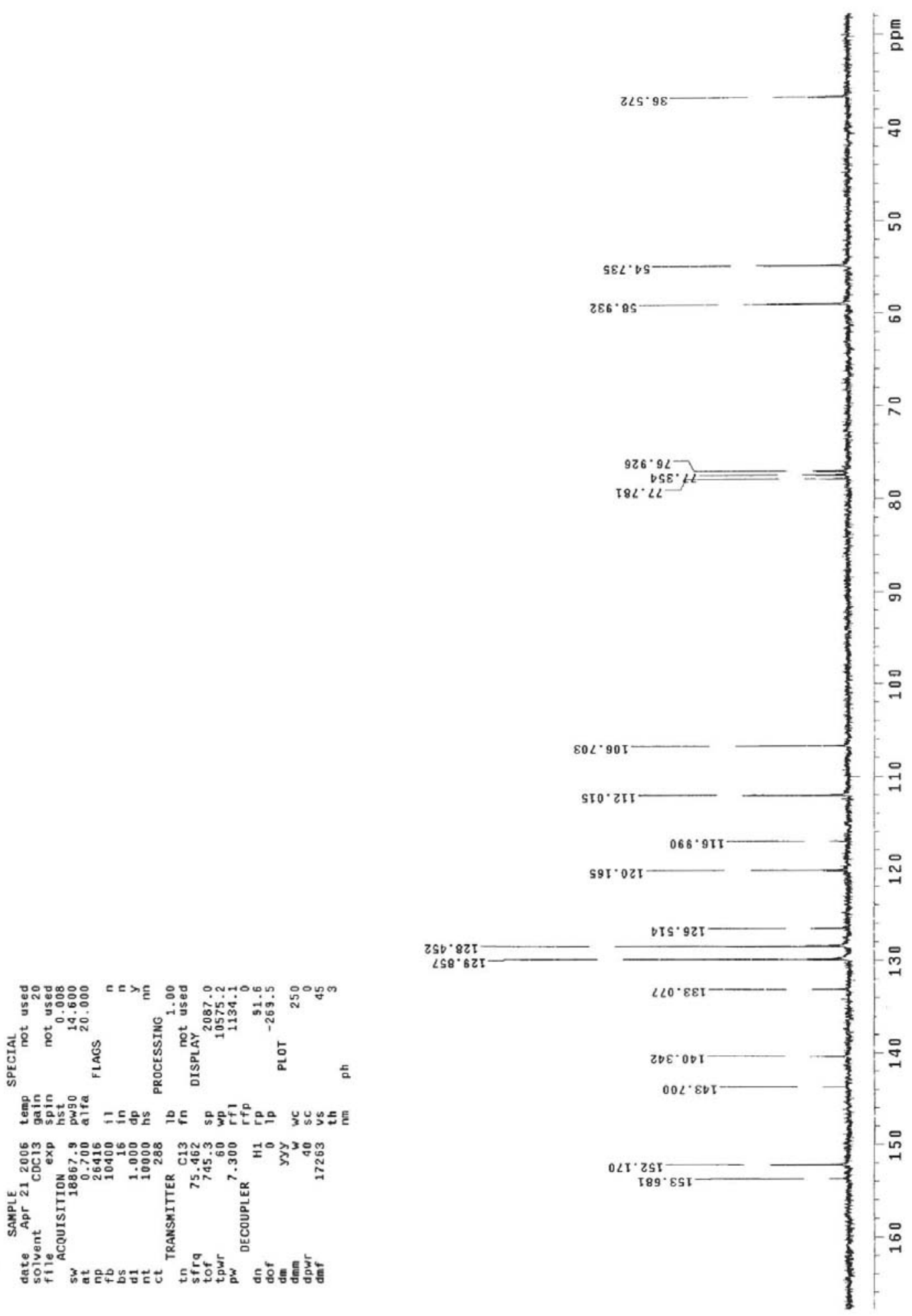

Fig. S-69: ${ }^{13} \mathrm{C}$ Spectra of 1-chloro-5,6-dihydro-6-[(methylamino)methyl]-5-(4-chloro phenyl)pyrrolo[1,2-f]pteridine $5.14 \mathrm{~b}$. 


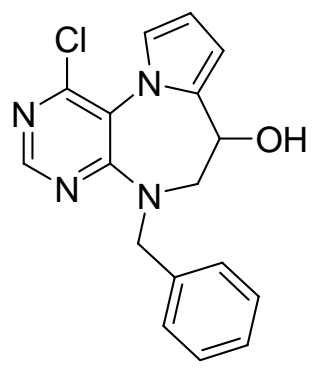

$$
\mathrm{C}_{17} \mathrm{H}_{15} \mathrm{ClN}_{4} \mathrm{O}
$$

Exact Mass: 326.09

6a
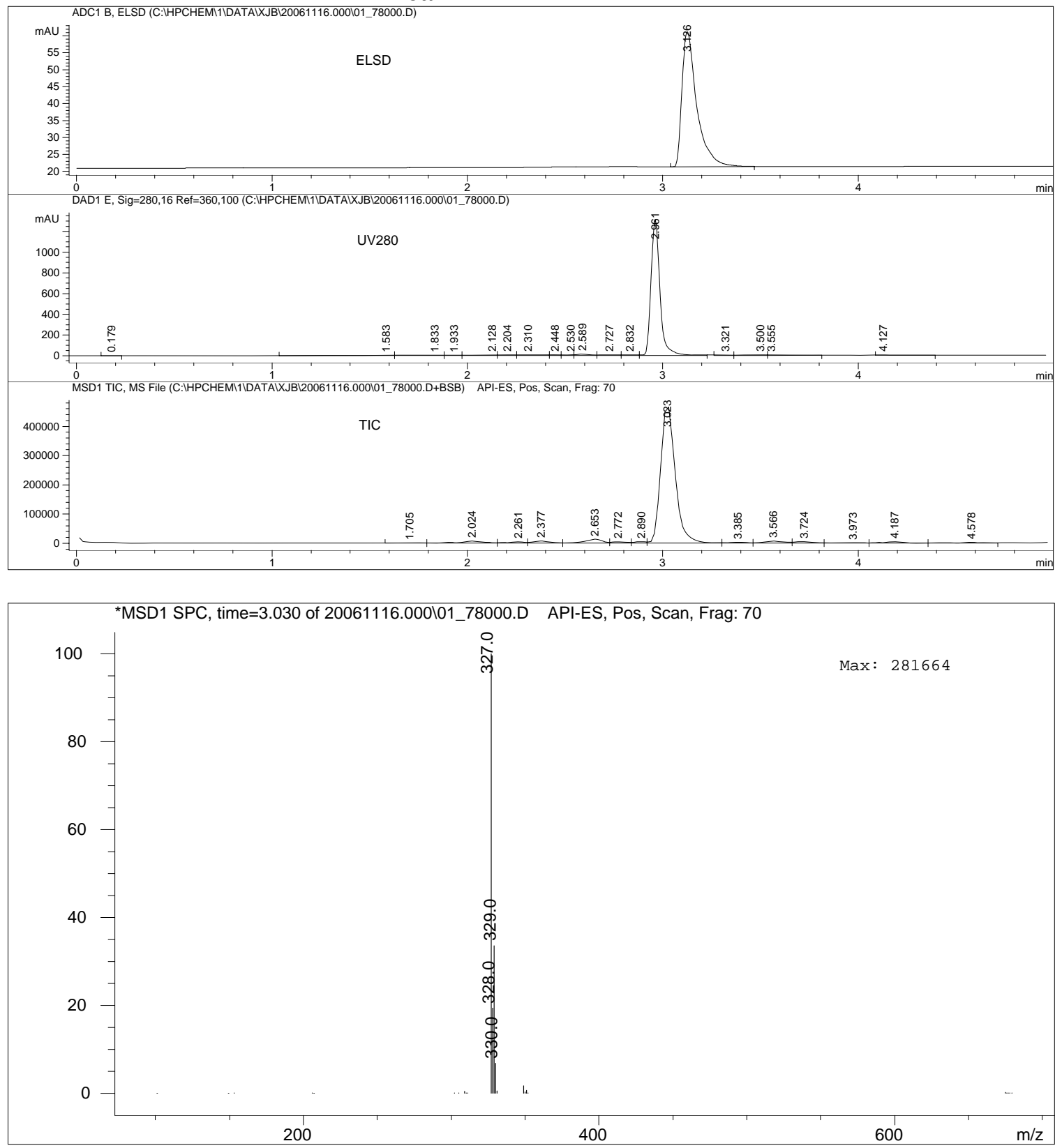

Fig. S-70: LC-MS-ELSD of 5-benzyl-1-chloro-6,7-dihydro-7-hydroxylpyrrolo[2,1-d] $[1,5]$ pyrimidodiazepine $6 a$. 

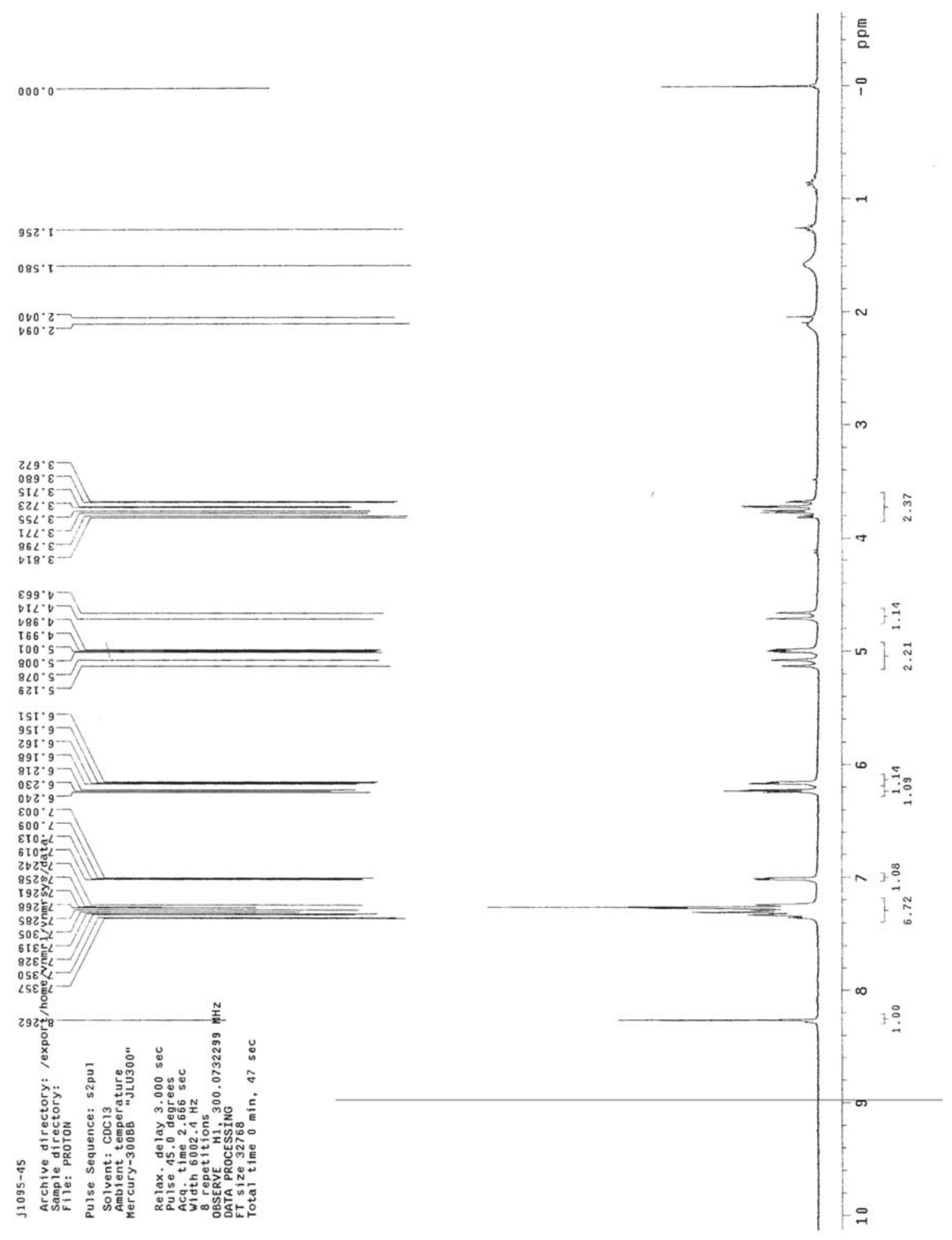

Fig. S-71: ${ }^{1} \mathrm{H}$ Spectra of 5-benzyl-1-chloro-6,7-dihydro-7-hydroxylpyrrolo[2,1-d] [1,5]pyrimidodiazepine 6a. 


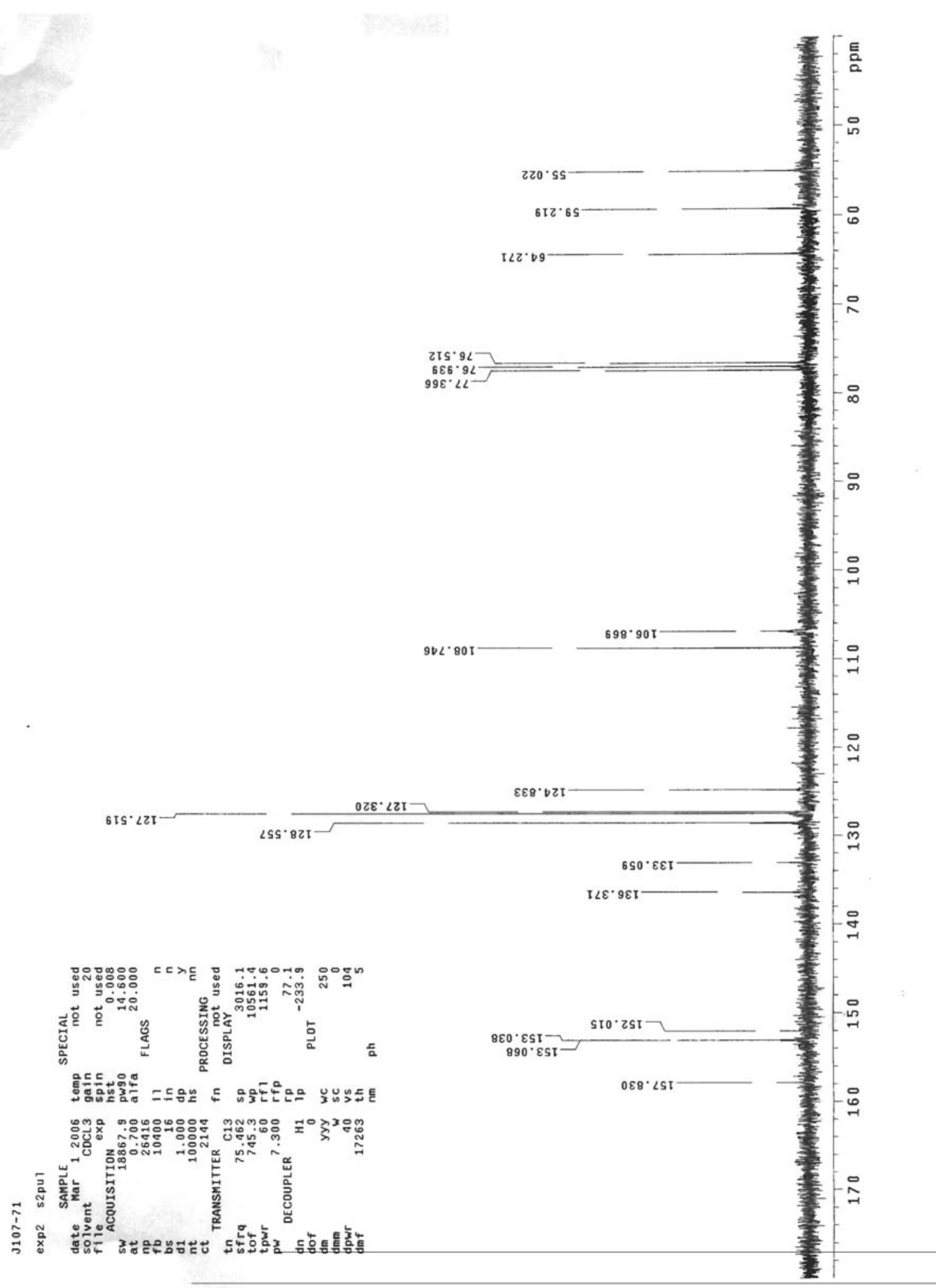

Fig. S-72: ${ }^{13} \mathrm{C}$ Spectra of 5-benzyl-1-chloro-6,7-dihydro-7-hydroxylpyrrolo[2,1-d] [1,5]pyrimidodiazepine 6a. 